\title{
Mode-Coupling Model of Mott Gap Collapse in the Cuprates: Natural Phase Boundary for Quantum Critical Points
}

\author{
R.S. Markiewicz \\ Physics Department, Northeastern University, Boston MA 02115, USA
}

A simple antiferromagnetic approach to the Mott transition was recently shown to provide a satisfactory explanation for the Mott gap collapse with doping observed in photoemission experiments on electron-doped cuprates. Here this approach is extended in a number of ways. RPA, mode coupling (via self-consistent renormalization), and (to a limited extent) self-consistent Born approximation calculations are compared to assess the roles of hot-spot fluctuations and interaction with spin waves. When fluctuations are included, the calculation satisfies the Mermin-Wagner theorem (Néel transition at $T=0$ only - unless interlayer coupling effects are included), and the mean-field gap and transition temperature are replaced by pseudogap and onset temperature. The model is in excellent agreement with experiments on the doping dependence of both photoemission dispersion and magnetic properties. The magnetic phase terminates in a quantum critical point (QCP), with a natural phase boundary for this QCP arising from hot-spot physics.

Since the resulting $\mathrm{T}=0$ antiferromagnetic transition is controlled by a generalized Stoner factor, an ansatz is made of dividing the Stoner factor up into a material-dependent part, the bare susceptibility and a correlation-dependent part, the Hubbard U, which depends only weakly on doping. From the material dependent part of the interaction, it is possible to explain the striking differences between electron- and holedoping, despite an approximate symmetry in the doping of the QCP. The slower divergence of the magnetic correlation length in hole doped cuprates may be an indication of more Mott-like physics.

Discussions of interlayer coupling, doping dependence of $U$, extension to a three-band model, and polaronic effects are included.

\section{INTRODUCTION}

Schrieffer, Wen, and Zhang ${ }^{1}$ originally proposed that the magnetic insulating phase in underdoped cuprates could be understood via a spin density wave (SDW) approach to the Mott transition, and successfully described the spin wave spectrum of the undoped parent compound, which is an antiferromagnetic (AFM) insulator. Kampf and Schrieffer ${ }^{2}$ showed that precursors of the Mott transition could give rise to a pseudogap in the quasiparticle spectrum, between incipient upper and lower Hubbard bands (U/LHBs). Attempts were quickly made to go beyond mean field theories by incorporating fluctuation effects, but a number of problems soon arose. While some calculations found evidence for pseudogaps ${ }^{3}$, others did not ${ }^{4}$.
The rapid disappearence of Neel order with hole doping created more problems: many calculations, even including strong fluctuations, predicted magnetic order as $T \rightarrow 0$, coupled with diverging magnetic correlation length $\xi$, whereas $\xi$ is found to remain finite even in the presence of the pseudogap. This has been used as evidence that the band structure picture of the Mott transition breaks down, and must be replaced by a local picture: 'Mott physics' instead of 'Slater physics'. On the other hand, other calculations find evidence for instabilities - either to incommensurate magnetism ${ }^{5}$ or to phase separation ${ }^{6,7}$, and the saturation of $\xi$ could be due to nanoscale phase separation physics. The situation is at a stalemate, with some models neglecting both phase separation and magnetic effects, and explaining the pseudogap in terms of purely superconducting precursor effects, while others find a magnetic quantum critical point (QCP) in the deeply underdoped regime, and yet others find a QCP above optimal doping.

Clearly, a simpler alternative is an important desideratum, and one has recently been proposed. While phase separation is a significant complication for hole doping, this instability appears to be greatly reduced or absent in electron-doped materials ${ }^{8,9}$, allowing a much simpler analysis. Moreover, for electron doping, the band picture involving short-range commensurate AFM order seems justified, in that magnetic correlations remain commensurate, while the correlation length diverges for all dopings up to the QCP. The desirability of a reference system free of phase separation complications coheres with Laughlin and Pines' observation ${ }^{10}$ : "This problem [of identifying the correct quantum protectorate] is exacerbated when the principles of self-organization ... compete. ... [H]igher organizing principles are best identified in the limiting case in which the competition is turned off, and the key breakthroughs are almost always associated with the serendipitous discovery of such limits."

While many models attempt to describe the properties of the cuprates over a limited doping range, it has proven difficult to systematically reproduce the changes over an extended doping range. Remarkably, simple mean field calculations ${ }^{9}$ were able to reproduce the full doping dependence of ARPES spectra in the electron-doped cuprates $^{8}$ in terms of a Mott gap collapse (QCP) near optimal doping. Here, these results are expanded upon in a number of ways. First, a number of models are applied to the electron-doped system, to see the effects of various correlations. A key issue is finite temperature effects: the RPA predicts a Neel temperature $T_{N} \sim U-$ much larger than found experimentally. Proper inclusion 
of thermal fluctuations, introduced via a self-consistent renormalization $(\mathrm{SCR})$ model $^{11,12}$, drives the Néel temperature $T_{N}$ to zero (Mermin-Wagner theorem), replacing the RPA gap $\Delta_{m f}$ by a SCR pseudogap $\Delta^{*}$ and the mean-field Néel temperature $T_{N}^{m f}$ with a crossover temperature $T^{*}$, with $\Delta_{m f} \simeq \Delta^{*}, T_{N}^{m f} \simeq T^{*}$. Inclusion of spin wave scattering, via the self-consistent Born approximation (SCBA) produces a large incoherent background, but the coherent part of the spectrum is recognizably the same as the spectrum found in the RPA and SCR approaches, with only moderate band renormalizations. The present conclusions (including Ref. 12) are consistent with more recent findings ${ }^{13,14}$.

In summary, there is a QCP near optimal doping in the electron-doped cuprates, associated with Mott (pseudo)gap collapse. The transition is characterized by three concurrent factors: termination of a zerotemperature AFM transition (which can be associated with a finite-T Néel transition due to weak interlayer coupling); collapse of a pseudogap centered on $(\pi, \pi)$; and crossover of the Fermi surface from small pockets to large barrel. Good agreement with experiment requires a weak Kanamori-style ${ }^{15}$ renormalization of the Hubbard $U$ with doping. The same model can describe both ARPES and magnetization results. A similar QCP is predicted at a comparable hole doping - indeed a natural phase boundary for magnetism exists, associated with hot spot physics. There is however, a striking difference in the hole doping case: saturation of a spin sum rule leads to much smaller correlation lengths and absence of finite-T Néel order. Stripe physics appears to play a lesser role - turning on at lower temperatures - possibly as a form of interaction of the doped polarons.

This paper is organized as follows. Section II describes the SCR formalism. Related Appendices discuss the extension to a three band model (Appendix A), the doping dependence of $U$ (Appendix B), and a more accurate solution of the self-consistency equation (Appendix C). Since the transition occurs when a Stoner factor equals unity, it is controlled by the real part of the bare susceptibility. Hence Section III reviews the properties of $R e \chi$, showing that plateaus in $\chi$ as a function of doping, $\vec{q}$, or $\omega$ are all controlled by the physics of hot spots. In turn, these plateaus provide natural phase boundaries for QCPs. The resulting susceptibility has a form similar to that postulated for a nearly antiferromagnetic Fermi liquid (NAFL), but a calculation of the NAFL parameters (Appendix D) finds that there are extra (cutoff) parameters, which cannot be neglected. In Section IV, this renormalized susceptibility is incorporated into the lowest-order correction to the electronic self energy, allowing a calculation of the spectral function associated with the pseudogap $\left(T_{N}=0\right)$. Excellent agreement is found with the ARPES spectra of $\mathrm{Nd}_{2-x} \mathrm{Ce}_{x} \mathrm{CuO}_{4 \pm \delta}$ (NCCO). A remaining problem lies in magnetic polaron effects which are expected at very low dopings: these bear some resemblance to nanoscale phase separation, and can lead to anomalous localization effects. Section $\mathrm{V}$ offers a brief introduction to these effects, by analyzing the self-consistent Born approximation at half filling. It is found that only minor quantitative changes to the earlier results are expected. An extension of the results to the hole-doped regime is considered in Section VI. The model also provides a good description of magnetic properties, as discussed in Section VII. Section VIII shows that inclusion of interlayer hopping leads to a finite $T_{N}$ (Appendix E). Results are discussed in Section IX, and Conclusions in Section X. Some of these results have been reported previously in the discussion of the mean-field results ${ }^{9}$ and in a conference procedings ${ }^{12}$.

\section{MODE-COUPLING CALCULATION}

\section{A. Model Dispersion and Doping Dependence of $U$}

In the present paper the mean field results are extended by incorporating fluctuations via mode-coupling theory ${ }^{16}$, following Moriya's self-consistent renormalization (SCR) $)^{11,17,18}$ procedure. Mode coupling theories have been applied to charge density wave (CDW) systems ${ }^{19,20}$, and have led to a successful theory of weak itinerant magnetic systems ${ }^{11,17}$. They have also been used to study glass transitions ${ }^{21}$, and recently extended to glasses in cuprates $^{22}$. The mode coupling analysis is particularly convenient, being the simplest model for which the Mermin-Wagner theorem is satisfied. The resulting pseudogaps compare well with recent photoemission experiments in electron-doped cuprates. While the SCR technique can be generalized to deal with competing phases ${ }^{23}$, only the antiferromagnetic fluctuations will be treated here.

The cuprates are treated in a one-band model. By comparison with a 3-band model (Appendix A), this can be shown to be an excellent approximation for the magnetic properties. The bare electronic dispersion is

$$
\epsilon_{k}=-2 t\left(c_{x}+c_{y}\right)-4 t^{\prime} c_{x} c_{y}
$$

with $c_{i}=\cos k_{i} a$. The dispersions for undoped $\mathrm{Sr}_{2} \mathrm{CuO}_{2} \mathrm{Cl}_{2}$ (SCOC) and electron-doped NCCO can be fit by assuming $t=0.326 \mathrm{eV}, t^{\prime} / t=-0.276$, with $U$ taken as an effective doping dependent parameter ${ }^{9}$, with $U=6 t$ at half filling. Similar parameters are found ${ }^{24}$ to describe the spin wave spectrum ${ }^{25}$ in $\mathrm{La}_{2} \mathrm{CuO}_{4}: t=$ $0.34 \mathrm{eV}, t^{\prime} / t=-0.25$, and $U / t=6.2$. The former values will be used here.

Many textbooks on strong correlation physics ${ }^{26,27}$ note that the Hubbard $U$ should be doping dependent, based on the original results of Kanamori ${ }^{15}$, but there are no satisfactory results for the doping dependence in the cuprates. A simple model calculation, which gives semiquantitative agreement with experiment in $\mathrm{NCCO}^{8,9}$, is described in Appendix B. 


\section{B. Self-Consistent Equation}

The SCR scheme is introduced to incorporate strong fluctuations near the antiferromagnetic wave vector $\vec{Q}$. The (path integral) formalism is standard ${ }^{27}$ and only the main results are given here. The quartic Hubbard contribution to the Hamiltonian is decoupled by a HubbardStratonovich transformation introducing spin wave fields $\phi$. The Fermion fields are then integrated out, leaving an approximate quartic effective action, which describes fluctuations about the mean field solution due to mode coupling. In the SCR model, the dynamical susceptibility is found self-consistently as

$$
\chi\left(\vec{q}, i \omega_{n}\right)=\frac{\chi_{0}\left(\vec{q}, i \omega_{n}\right)}{1-U \chi_{0}\left(\vec{q}, i \omega_{n}\right)+\lambda},
$$

with the bare susceptibility

$$
\chi_{0}(\vec{q}, \omega)=-\sum_{\vec{k}} \frac{f\left(\epsilon_{\vec{k}}\right)-f\left(\epsilon_{\vec{k}+\vec{q}}\right)}{\epsilon_{\vec{k}}-\epsilon_{\vec{k}+\vec{q}}+\omega+i \delta},
$$

where $\delta$ is a positive infinitesimal, and the RPA susceptibility given by Eq. 2 with $\lambda=0$.

The leading divergence corresponds to AFM at $\vec{q}=\vec{Q}$, so the denominator of Eq. 2 - the (inverse) Stoner factor - is expanded in terms of the small parameters $\omega$ and $\vec{q}^{\prime} \equiv \vec{q}-\vec{Q}$ (analytically continuing $\left.i \omega_{n} \rightarrow \omega+i \epsilon\right)$ :

$$
\delta_{q}(\omega)=1-U \chi_{0}(\vec{q}, \omega)+\lambda=\delta+A q^{2}-B \omega^{2}-i C \omega,
$$

where

$$
\delta=1-U \chi_{0}(\vec{Q}, 0)+\lambda,
$$

and $\delta_{0}=\delta-\lambda$. The self-consistent equation for $\delta$ is

$$
\delta=\delta_{0}+\frac{12 u}{\beta V} \sum_{\vec{q}, i \omega_{n}} D_{0}\left(\vec{q}, i \omega_{n}\right)
$$

where $u$ is a measure of the quartic mode-mode coupling (Appendix D4) and

$$
D_{0}^{-1}\left(\vec{q}, i \omega_{n}\right)=\delta+A q^{\prime 2}+C\left|\omega_{n}\right|
$$

The sum over Matsubara frequencies can be carried out using

$$
\begin{aligned}
\frac{1}{\beta} \sum_{i \omega_{n}} X\left(i \omega_{n}\right) & =-\frac{1}{\beta \pi} \sum_{i \omega_{n}} \int_{-\infty}^{\infty} d \epsilon \frac{\operatorname{Im} X(\epsilon+i \delta)}{i \omega_{n}-\epsilon} \\
& =-\int_{0}^{\infty} d \frac{\epsilon}{\pi} \operatorname{coth} \frac{\epsilon}{2 T} \operatorname{Im} X(\epsilon+i \delta) .
\end{aligned}
$$

Then

$$
\begin{array}{r}
\frac{1}{\beta V} \sum_{\vec{q}, i \omega_{n}} D_{0}\left(\vec{q}, i \omega_{n}\right) \\
=\int \frac{d^{2} \vec{q} a^{2}}{(2 \pi)^{2}} \int_{0}^{\alpha_{\omega} / C} \frac{d \epsilon}{\pi} \operatorname{coth} \frac{\epsilon}{2 T} \frac{C \epsilon}{\left(\delta+A q^{\prime 2}\right)^{2}+(C \epsilon)^{2}} .
\end{array}
$$

Note the sharp energy cutoff in Eq. 9. This comes about because the linear-in- $\omega$ dissipation is a result of Landau damping of the spin waves by electrons near the hot spots, and therefore the dissipation cuts off when the spin wave spectrum gets out of the electron-hole continuum. The cutoff parameter $\alpha_{\omega}$ is defined in Appendix D2, above Eq. D10. Numerical calculations (Fig. 40) show that the cutoff can be quite sharp, particularly near the VHS.

\section{Approximate Solutions}

Equations 6, 9 can easily be solved in the limit $T=0$. In this case, there is a transition at

$$
\begin{gathered}
\delta_{0}=-12 u \int_{0}^{q_{c}^{2}} \frac{d q^{2} a^{2}}{4 \pi} \int_{0}^{\alpha_{\omega} / C} \frac{d \epsilon}{\pi} \frac{C \epsilon}{\left(A q^{2}\right)^{2}+(C \epsilon)^{2}} \\
=-\frac{3 u q_{c}^{2} a^{2}}{\pi^{2} C} R_{0} \equiv 1-\eta \\
R_{0}=\frac{1}{2} \ln \left[1+a_{q}^{-2}\right]+\frac{\tan ^{-1}\left(a_{q}\right)}{a_{q}}
\end{gathered}
$$

with $a_{q}=A q_{c}^{2} / \alpha_{\omega}$. Since the right-hand side is finite and negative, fluctuations reduce but in general do not eliminate the order at $T=0$. At the $\operatorname{RPA}$ level $(\lambda=0)$, the AFM instability is controlled by the Stoner criterion, $\delta_{0} \rightarrow 0$. The quantum corrected Stoner criterion is $U \chi_{0}=\eta$, where representative values of $\eta$ are listed in Table I.

However, for finite $T$, there are corrections $\sim \ln (\delta)$, so $\delta$ cannot be set to zero, and there is no finite temperature transition (the Mermin-Wagner theorem is satisfied). To see this, it is adequate to approximate $\operatorname{coth}(x)$ as $1 / x$ for $x \leq 1$ and 1 for $x>1$. In this case, Eq. 6 can be solved exactly, Appendix C. However, this exact solution is not very illuminating, and a simpler approximate solution will be given here. Since only the term proportional to $T$ is singular, $T$ and $\delta$ can be set to zero in the remaining term. Defining

$$
\bar{\delta}_{0}=\delta_{0}+\eta-1
$$

Eq. 6 becomes

$$
\begin{aligned}
\delta-\bar{\delta}_{0}=\frac{6 u T a^{2}}{\pi^{2} A} \int_{\delta}^{\delta+} & \frac{A q_{c}^{2}}{y} \tan ^{-1}\left(\frac{2 T C}{y}\right) \\
& \simeq \frac{3 u T a^{2}}{\pi A} \ln \left(\frac{2 C T}{\delta}\right),
\end{aligned}
$$


where the second line uses Eq. C6, below. Hence, there is no finite temperature phase transition, and $\delta$ only approaches zero asymptotically as $T \rightarrow 0$ : approximately,

$$
\delta=2 C T e^{-\pi A\left|\bar{\delta}_{0}\right| / 3 u T a^{2}} .
$$

\section{Susceptibility}

Given the (inverse) Stoner factor $\delta_{q}$, Eq. 4, the renormalized susceptibility can be written in nearlyantiferromagnetic Fermi liquid (NAFL) ${ }^{28}$ form,

$$
\chi(\vec{q}, \omega)=\frac{\chi_{Q}}{1+\xi^{2}(\vec{q}-\vec{Q})^{2}-\omega^{2} / \Delta^{2}-i \omega / \omega_{s f}},
$$

with coefficients

$$
\begin{gathered}
\chi_{\vec{Q}}=\frac{\chi_{0}}{\delta} \\
\xi^{2}=\frac{A}{\delta} \\
\Delta^{2}=\frac{\delta}{B} \\
\omega_{s f}=\frac{\delta}{C}
\end{gathered}
$$

The similarity of Eq. 15 to the corresponding result for CDW's ${ }^{20}$ should be noted - the SCR is a form of mode coupling theory.

In the renormalized classical regime, the vanishing of $\delta$ as $T \rightarrow 0$ is controlled by a correlation length, Eq. 17, which can be written as ${ }^{29}$

$$
\xi=\xi_{0} e^{2 \pi \rho_{s} / k_{B} T} .
$$

Numerically solving Eq. 13 (or Eq. C7) for $\delta$, then the spin stiffness $\rho_{s}$ is exactly given by

$$
\rho_{s}=\frac{k_{B} T}{4 \pi} \ln \left(\frac{A}{\xi_{0}^{2} \delta}\right),
$$

with $\xi_{0}=\sqrt{\frac{e A}{2 T C}}$. Using Eq. 14, an approximate $\rho_{s}$ is:

$$
\rho_{s}^{a}=\frac{A\left|\bar{\delta}_{0}\right|}{12 u a^{2}} .
$$

$\rho_{s}$ is plotted in Fig. 11b, with $u^{-1}=0.384 \mathrm{eV}$, chosen to give a $\rho_{s}$ in agreement with experiment for $x=0$, $T=0$ (Section VII). The T-dependence of the prefactor $\xi_{0}$ agrees with one-loop $\sigma$-model results ${ }^{30}$ rather than the more accurate two-loop results ${ }^{29,31}$. This difference is presumably a deficiency of the present model in not using fully self consistent parameters; it will be discussed further in Section VII.

\section{E. Parameter Evaluation}

The susceptibility Eq. 15 is well-known in NAFL ${ }^{28,32}$ and spin fermion ${ }^{33,34}$ theories and in renormalization group (RG) calculations of quantum phase transitions ${ }^{35,36}$. In these calculations, the parameters of Eq. 15 (equivalently, $A, B$, and $C$ ) are usually determined empirically from fits to experiments. However, the good agreement between experiment and mean field theory for electron doped cuprates encourages us to try to calculate these parameters from first principles, following Ref. 17, in terms of a renormalized Hubbard parameter $U_{\text {eff }}$ and a mode coupling parameter $u$. This puts a special premium on the bare susceptibility $\chi_{0}$, which is assumed to control the main material, doping, and pressure dependence of the Mott transition, while the interaction parameters $U$ and $u$ are relatively constant. In fact, it is found that $U$ has a weak but important doping dependence, estimated in Appendix B, which is consistent with experiment. A single, doping-independent value of $u$ is chosen to agree with $t-J$ results at half filling.

The motivation for this approach comes from experience with another strongly correlated system: electronhole droplets in photoexcited semiconductors. Here it was found ${ }^{37,38}$ that the correlation effects were controlled by an isotropic density-dependent interaction potential, whereas the material, anisotropy, and uniaxial pressure dependence were controlled by the kinetic energy - i.e., by the bare band structure. A similar approach was applied to CDW systems ${ }^{39}$.

\section{HOT SPOT PLATEAUS AND GENERIC QCPS}

While the properties of $\chi_{0}$ are now reasonably well understood, they remarkably do not seem to have been used to derive the parameters of SCR or NAFL theory. Here this oversight is corrected. In particular, calculation of the curvature parameter $A$ is discussed below. A new cutoff parameter $q_{c}$ is introduced, which is essential in explaining the differences between the QCPs for hole and electron dopings. The corresponding frequency parameter $C, \mathrm{Eq} \mathrm{D} 7$, and its associated cutoff parameter $\alpha_{\omega}$ (below Eq D10) are discussed in Appendix D2. 


\section{A. Plateaus in Doping Dependence}

\section{Hot Spots}

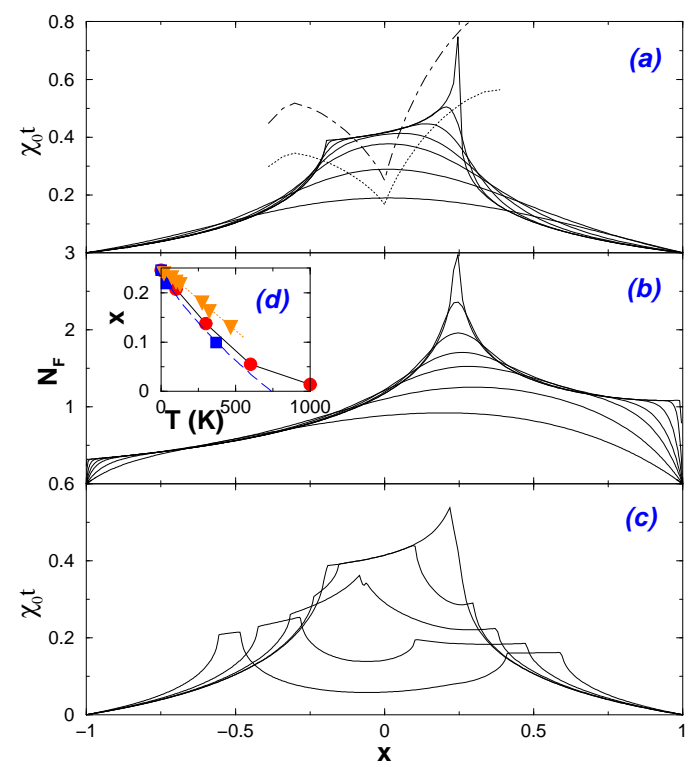

FIG. 1. (a) Susceptibility $\chi_{0}$ at $\vec{Q}$ as a function of doping for several temperatures. From highest to lowest curves near $x=0.1$, the temperatures are $T=1,100,300,600,1000$, 2000 , and $4000 \mathrm{~K}$. Dotted line $=1 / U_{\text {eff }}$, dot-dashed line $=$ $1.5 / U_{\text {eff }}$. (b) Density of states $N_{F}$ for the same temperatures. (c) Susceptibility $\chi_{0}$ at $\vec{Q}$ as a function of doping for several frequencies at $T=1 K: \omega=0.01,0.1,0.3,0.6,1.0 \mathrm{eV}$. (d) Pseudo-VHS (peak of $\chi_{0}$ ) as a function of temperature $T_{V}$ (circles) or scaled frequency $T_{c}^{-}=\omega_{c}^{-} / \pi$ (squares); triangles $=T_{\text {incomm }}$.

In the self-consistent renormalization scheme, the $T=$ 0 AFM transition is controlled by a Stoner factor, $U R e\left(\chi_{0}\right)=\eta$, where $\eta>1$ includes a quantum correction, Table I. Hence, the relevant quantity on which the study is based is the real part of the bare magnetic susceptibility, Eq. 3. This susceptibility has been analyzed in a number of papers. Whereas usually only $\operatorname{Im}(\chi)$ is explored in detail (e.g., Refs. 40-42), $R e(\chi)$ was studied in Ref. 43. The extended discussion which follows is intended to bring out salient features for the computation of the NAFL parameters.

The doping dependence of $\chi_{0}(\vec{Q}, \omega)$ is illustrated in Fig. 1a, where $\vec{Q}=(\pi, \pi)$. At low $T$, the susceptibility has a plateau shape, which is not present in the density of states, $N_{F}$, Fig. 1b. Beyond the plateau edges $\chi_{0}$ falls off sharply on both electron and hole doping sides of half filling. This sharp falloff explains the appearence of QCPs: the Stoner criterion is satisfied on the plateau, but fails when $\chi_{0}$ drops.

The plateau shape is characteristic of hot spot physics. Hot spots are those points where the Fermi surface (FS) intersects the replica FS shifted by $\vec{Q}$. They are located at $c_{x}=-c_{y}=c_{x 0}$, with

$$
c_{x 0}=\cos a k_{x 0}=\sqrt{\frac{\mu}{4 t^{\prime}}},
$$

and equivalent points. The edges of the plateau are those points at which the overlap terminates (hot spots cease to exist). For the present band structure, hot spots exist only when the chemical potential $\mu$ is in the range $4 t^{\prime} \leq$ $\mu \leq 0$, or for doping $0.25>x>-0.19$ (electron dopings are considered as negative). Since the two end points play an important role, it is convenient to label them, and they are here called 'hot' hot spot and 'cold' hot spot (or H-point and C-point) for the hole and electron-doped termination points, respectively. It will be demonstrated below that at each doping, the hot spots also lead to a susceptibility plateau in momentum space, around $\vec{Q}$, collapsing to a logarithmic (square root) divergence at the H- (C-)point. The $H$-point is the VHS, and hence also involves a conventional ETT. The physics is simpler near the $C$-point, where the topology hardly changes but the FS and $\vec{Q}$-FS become decoupled (it is therefore a form of Kohn anomaly ${ }^{43}$ ).

\section{Mean Field Mott Transition}

For the parameter values expected in the cuprates, these susceptibility plateaus control the physics of the Mott gap collapse. As a function of doping, the mean field Mott gap is found to close at a doping just beyond the edge of the plateau, for both electron and hole doping, Fig. 2. The solid and long dashed lines are the commensurate and incommensurate mean field Mott transition temperatures $T^{*}(x)$ calculated using the estimated $U_{\text {eff }}(x)$, dotted line in Fig. 1. For electron doping, there is a double transition, first from commensurate to incommensurate antiferromagnetic order at the plateau edge, then to the loss of any magnetic order at a slightly higher doping (inset a). For hole doping, the dominant antiferromagnetic order is incommensurate for all dopings, but the difference in $T_{N}$ becomes significant only near the $\mathrm{H}$ point (inset b). When fluctuations are included (below), it is found that the Néel transition is shifted to zero temperature, while a pseudogap first appears near the mean field $T_{N}$. Note that in the hole doped regime, there is good agreement between the mean field transition and the pseudogap (squares in Fig. $1 \mathrm{~b}=$ data of Krasnov ${ }^{44}$, assuming $\left.2 \Delta=4.6 T^{*}\right)$. For the real cuprates, the terminations of the Mott gaps are preempted by superconducting transitions, close to the critical regime. 


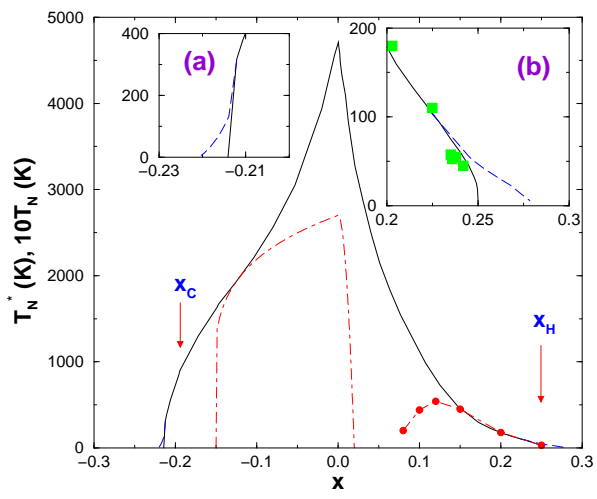

FIG. 2. Mean field magnetic transition temperatures determined from Stoner criterion using $U_{\text {eff }}$ of Fig. 1. Solid line: commensurate (at $\vec{Q}$ ); long dashed line: incommensurate. Dot-dashed line $=10 T_{N}$, where $T_{N}$ is the onset of long range AFM order, from [ 45] and [46] (with filled circles). Insets = blowups near C- and H-points. Squares in inset b = pseudogap data of [44].

\section{The Pseudo-VHS}

The susceptibility Fig. 1a has a remarkable doping dependence, with the large peak at the Van Hove singularity (VHS) shifting ${ }^{43}$ to half filling with increasing temperature $T$. The peak position of this 'pseudo-VHS' defines a temperature $T_{V}(x)$, Fig. 1d (circles). This behavior can readily be understood from the form of $\chi_{0}(\vec{Q}, 0)$, Eq. 3 . The denominator $\epsilon_{\vec{k}}-\epsilon_{\vec{k}+\vec{Q}}=-4 t\left(c_{x}+c_{y}\right)$, is independent of $t^{\prime}$, and hence has a stronger divergence than the density of states (dos). Indeed, this divergence matches the strong VHS found for $t^{\prime}=0$ (perfect nesting), and like that VHS falls at half filling, $x=0$. There is one crucial difference - at low temperatures, this divergence is cut off by the Fermi functions, which leave the integrand non zero in a wedge which intercepts the zone diagonal (where the denominator vanishes) only at isolated points: the hot spots. Hence, the residual divergence at low $T$ is still dominated by the conventional VHS. However, at finite $T$, excitations along the zone diagonal become allowed, leading to a stronger divergence of $\chi_{0}(\vec{Q}, 0)$ near $x=0$.

The strong temperature dependence of the pseudoVHS is in strong contrast to the density of states, $N_{F}$, Fig. 1 b, and also with the pairing correlations ${ }^{43}$. The denominator of the pairing susceptibility involves the sum of the energies, $\epsilon_{\vec{k}}+\epsilon_{\vec{k}+\vec{Q}}=-8 t^{\prime} c_{x} c_{y}$, rather than their difference (as in Eq. 3), and hence always peaks at the ordinary VHS.

The difference between nesting and pairing susceptibilities has a fundamental significance. By mixing electron and hole-like excitations, the superconducting gap is always pinned to the Fermi level, and can open up a full gap at any doping. On the other hand, a nesting gap need not be centered on the Fermi surface, and is constrained to obey Luttinger's theorem, conserving the net number of carriers in the resultant Fermi surface. Hence, the only way a nesting instability (such as antiferromagnetism) can open a full gap at the Fermi level is for the instability to migrate with increased coupling strength to integer filling of a superlattice zone (e.g., half filling of the normal state).

Since the susceptibility has such a distinct temperature dependence from the density of states, one might ask how the frequency dependence compares. This is illustrated in Fig. 1c at low temperature (1K). While the frequency introduces additional sharp features and has an overall very distinct appearence from the T-dependence, nevertheless the main peak also shifts from the VHS toward lower doping with increasing $\omega$ - in fact, the shift is almost the same when comparing $\hbar \omega$ and $\pi k_{B} T$, Fig. 1d. The dashed line in Fig. 1d is $T_{c}^{-}=\hbar \omega_{c}^{-} / \pi k_{B}$, with ${ }^{40}$

$$
\omega_{c}^{-}=\frac{4 t(\hat{\mu}-\tau)}{1-\tau},
$$

with $\tau=2 t^{\prime} / t$ and $\hat{\mu}=\mu / 2 t$. The proportionality of frequency and temperature dependences holds only in the hole doped regime: temperature shifts the susceptibility peak only to half filling, $x=0$, while frequency will shift the peak beyond half filling $(x<0)$.

The structure in the low temperature susceptibility, Fig. 1, with its largest peak at the $\mathrm{H}$-point on the hole doped side, is in striking contrast to the calculated doping dependence of the Néel transition, Fig. 2, which has a broad plateau on the electron-doped side, but falls off more quickly with hole doping, showing no sign of a peak near the VHS. This contrast can be accounted for by two effects. First, the shift of spectral weight with temperature of the pseudo-VHS, noted in Fig. 1, would tend to produce a symmetric falloff of $T_{N}$ with either electron or hole doping. But the dos peak at the VHS leads to better screening of $U_{\text {eff }}$ for hole doping, thereby further depressing $T_{N}$.

\section{Neel Transition}

The mean field Neel transition is associated with shortrange magnetic order, and hence should be compared to the experimental pseudogap transition $T^{*}$, while the experimental Neel transition involves long-range magnetic order. It is controlled by small parameters, such as anisotropy and interlayer coupling (Section VIII) and need have no connection to the mean field $T_{N}$. Nevertheless, the mean field calculation provides an approximate envelope of the resulting data, but overestimates the transition temperatures by a factor of 10, Fig. 2 . The agreement is particularly good on the electron doped side (except for overestimating the doping of the QCP), while for hole doping the experimentally observed ${ }^{45} T_{N}$ (dot-dashed line) shows a stronger falloff, perhaps due 
to phase separation. Since stripes can frustrate magnetic order, the figure also includes the magnetic ordering temperature of quasi-static stripe arrays, from $\mathrm{Nd}$ substituted $\mathrm{La}_{2-x} \mathrm{Sr}_{x} \mathrm{CuO}_{4}$ (LSCO) ${ }^{46}$, which is taken as a lower bound for the Néel ordering transition in the absence of stripes. A possible explanation for the rough proportionality of the mean field and long-range Néel transitions will be discussed in Section VIII.

\section{B. Plateaus in Momentum Space}

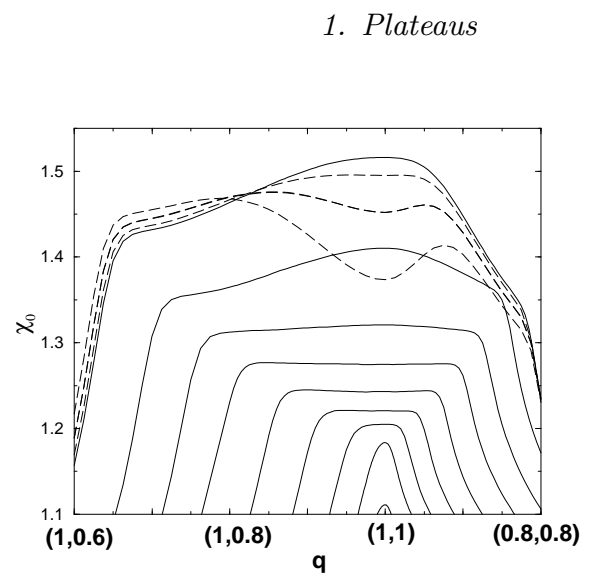

FIG. 3. Susceptibility $\chi_{0}$ near $\vec{Q}$ for a variety of dopings at $T=100 K$. From highest to lowest solid curves near $S \equiv \vec{Q}$, the chemical potentials are $\mu=-0.35,-0.30,-0.25,-0.20,-0.15$, $-0.10,-0.055,-0.02$, and $0 \mathrm{eV}$. For the dashed curves (top to bottom), $\mu=-0.352,-0.355$, and $-0.359 \mathrm{eV}$.

In analyzing either thermal fluctuations or the quantum fluctuations associated with QCPs, it is necessary to understand the susceptibility near the AFM vector $\vec{Q}$. At each doping, hot spot physics leads to a plateau in momentum space, centered on $\vec{Q}$. Figure 3 shows how $\chi_{0}$ varies near $\vec{Q}$ at a low temperature $(100 \mathrm{~K})$ for a series of different dopings. Results near $T=0$ are presented in Ref. 12. For all dopings there is a plateau in $q$. The width of the plateau at $T=0$ can be readily determined: in any direction, it is the minimum $q$ needed to shift the replica FS so that the hot spots are eliminated. This can be found from the dispersion, Eq. 1, by substituting $\vec{k} \rightarrow(\vec{Q}+\vec{q}) / 2$, or

$$
-2 t\left(\hat{s}_{x}+\hat{s}_{y}\right)-4 t^{\prime} \hat{s}_{x} \hat{s}_{y}=\mu
$$

with $\hat{s}_{i}=\sin \left(q_{i} a / 2\right)$. As shown in Fig. 4, this formula agrees with the (anisotropic) plateau width measured from Fig. 3 (circles). The inset shows the shape of the plateau as a function of doping. The diamond shape of the plateau, Eq. 25, is related to the profile of the hole pockets formed by the overlap of the shifted and unshifted FSs. Specifically, the plateau is the region of overlap of the two hole pockets, shifted to have a common center, as illustrated in Fig. 5. The remaining parts of the pockets also show up, as ridges ${ }^{47}$ in the susceptibility, radiating from the corners of the diamond (similar to the peaks in the $\mu=0.05 \mathrm{eV}$ data in Fig. 6, below). As noted by Bénard, et al. ${ }^{40}$, the susceptibility in two-dimensions acts as a FS caliper. The plateau width leads to a natural limit on the magnetic correlation length, $\xi_{c} \sim 1 / q_{c}$, in agreement with experimental data from $\mathrm{YBa}_{2} \mathrm{Cu}_{3} \mathrm{O}_{7-\delta}(\mathrm{YBCO})^{48,49}$ (squares, triangles in Fig. 4), as noted previously ${ }^{42,43}$. Related data from $\mathrm{LSCO}^{50}$ are also shown.

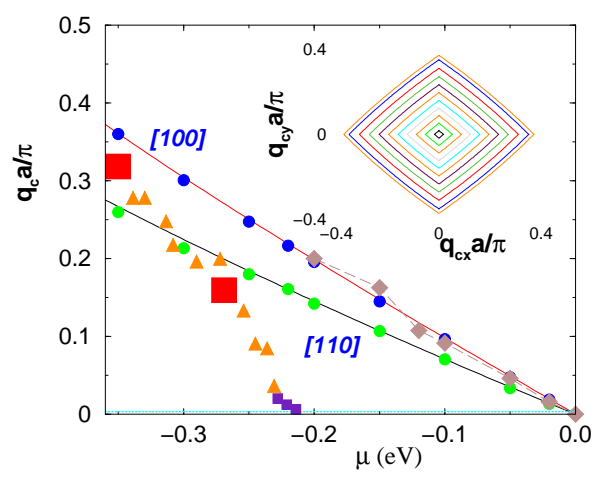

FIG. 4. Plateau width $q_{c}$, comparing Eq.25 (solid lines) and the measured widths (circles) from Fig. 3. Upper curve along $\left[q_{c}, 0\right]$ direction, lower along $\left[q_{c}, q_{c}\right] / \sqrt{2}$ direction. Symbols $=$ experimental inverse correlation lengths $\xi^{-1}$ from YBCO: large squares $=$ Ref. 48 , triangles $=$ Ref. 49; LSCO: small squares $=$ Ref. 50. Diamonds $=T_{A}^{*} / 5000 \mathrm{~K}$. Dotted line: $\xi=100 a$. Inset $=$ plateau boundary for a series of chemical potentials $\mu$ from 0 (smallest) to $-0.359 \mathrm{eV}$ (largest).

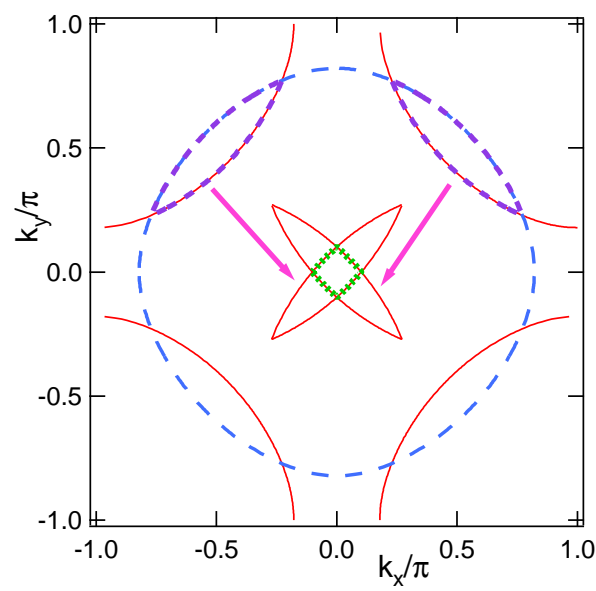

FIG. 5. Illustrating origin of plateaus (dotted line) from crossed hole pockets (short dashed lines). 


\section{Cusps}

For electron doping, the plateaus in $q$ are particularly flat topped, Fig. 3. At low temperatures the edges sharpen up Fig. 6 and the falloff in $\chi_{0}$ acquires a squareroot singularity (Appendix D1). The width of the plateau decreasing to zero as $x \rightarrow x_{C}$, and for electron-doping beyond the C-point $(\mu>0)$, the plateau ends and the susceptibility displays split peaks away from $\vec{Q}$, Fig. 6, with a dip in between. Thus $x_{C}$ is a $\mathrm{QCP}^{43}$ where the magnetic order changes from commensurate to incommensurate. (There is a corresponding QCP at the $\mathrm{H}$-point ${ }^{43}$.)

However, the magnitude of $\chi_{0}$ also changes rapidly near $\mu=0$, so there should be an independent QCP from a magnetic to a non-magnetic phase near the same doping, as discussed in the previous subsection (note the line depicting $1 / U(\mu=0)$ in Fig. 6$)$.

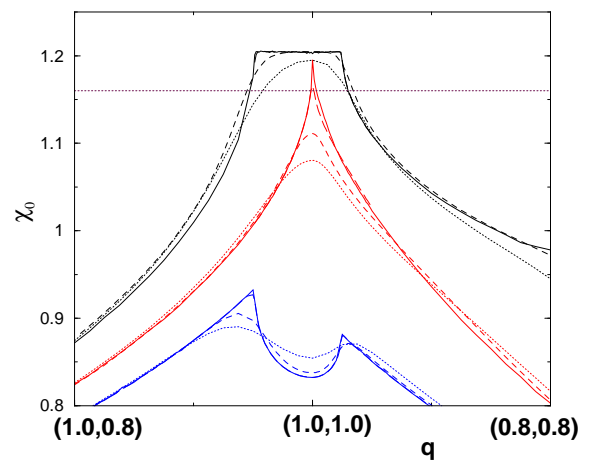

FIG. 6. Susceptibility $\chi_{0}$ near $\vec{Q}$ for several dopings near the C-point. Upper group at $\mu=-0.05 \mathrm{eV}$, middle at $\mu$ $=0$ (C-point), and bottom at $\mu=+0.05 \mathrm{eV}$. Temperatures are $T=200 K$ (dotted lines), $100 \mathrm{~K}$ (short dashed lines), $10 \mathrm{~K}$ (long dashed lines), $1 \mathrm{~K}$ (solid lines). Horizontal line = $U_{\text {eff }}(\mu=0)$.

Technically, similar cusps also arise at the plateau edges for electron doping, $0>\mu>-0.22 \mathrm{eV}$. The tops of the plateaus are not completely flat, Fig. 7a and the highest susceptibility is shifted away from $\vec{Q}$ (Appendix D1). However, these effects are much weaker than those associated with $\mu>0(\Delta \chi / \chi \leq 0.5 \%$ - compare the vertical scales of Figs. 6, 7). Thus near the mean-field transition any structure on the plateaus is smeared out by thermal broadening. Even at $T=0$, these features are likely to be negligible compared to dispersion in $U$ which arises from renormalization effects ${ }^{51}$.

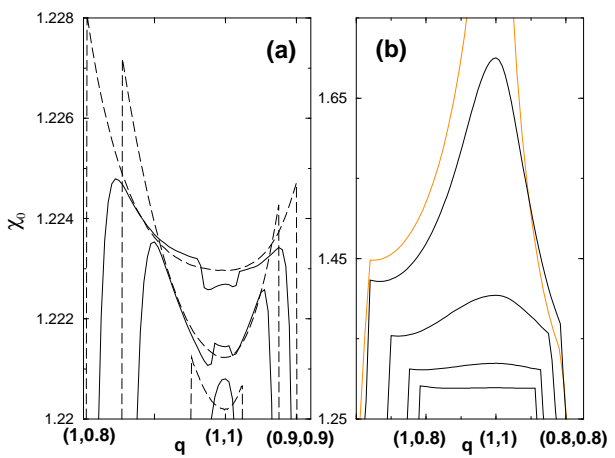

FIG. 7. (a): Expanded view of susceptibility $\chi_{0}$ on the plateaus near $\vec{Q}$ for a variety of dopings at $T=100 K$ (solid curves) or $1 \mathrm{~K}$ (dashed curves). From highest to lowest curves near $\vec{Q}$, the chemical potentials are $\mu=-0.20,-0.15$, and -0.05 $\mathrm{eV}$ (for both solid and dashed curves). All curves except $\mu=-0.20 \mathrm{eV}$ have been shifted vertically to fit within the expanded frame. (b): Similar plateaus for the hole doped materials $(T=1 K)$, with (from highest to lowest) $\mu=-0.359$, $-0.35,-0.3,-0.25$, and $-0.22 \mathrm{eV}$.

\section{Curvature (A)}

The plateau is a region of anomalously small local curvature $\hat{A}=A / U$ (Eq. 63) of the susceptibility, $\chi_{0}(\vec{Q}+\vec{q})=\chi_{Q}-\hat{A} q^{2}$, where $A$ is an important NAFL parameter. Clearly, at $T=100 K$ the curvature $A$ has gone negative near the $H$-point, Fig. 3 . At even lower temperatures, it reverts to positive values, Fig. 7b. The temperature dependence of the normalized parameter $A^{\prime}=(\pi / a)^{2}(A / t)$ is illustrated in Fig. 8 at several dopings. The temperature dependence is dominated by divergences at both $\mathrm{H}$ - and $\mathrm{C}$-points. The divergence at the H-point Fig. 8a is the well-known logarithmic VHS. However, at finite temperatures spectral weight is shifted away from the VHS and $A$ turns negative, only recovering a positive sign above $T \simeq 2000 K$. The temperature at which $A$ turns negative can be defined as $T_{\text {incomm }}: A<0$ for $T>T_{\text {incomm }}$. From Fig. 1d, $T_{\text {incomm }}$ is comparable to but larger than $T_{V}$ (for $x \leq 0.06 A$ remains positive). This in fact explains the origin of $T_{\text {incomm }}$. Figure 8 a demonstrates that $A$ is negative at $T \rightarrow 0$ beyond the H-point $(\mu=-0.4 e V)$. Thus, increasing $T$ above $T_{V}$ produces the same susceptibility crossover. A similar crossover was discussed by Sachdev, et al. ${ }^{52}$, except that they assumed that in the high temperature phase the AFM fluctuations remained centered on the commensurate $\vec{Q}$, whereas here $A$ is negative. At sufficiently high temperatures $A$ again becomes positive for all dopings i.e., the leading singularity of $\chi_{0}$ is always at $\vec{Q}$.

At the C-point, the collapse of the plateau width translates into a divergence of the curvature at $\vec{Q}(\hat{A} \rightarrow \infty)$. This divergence of the high-temperature susceptibility is cut off at low $T$, Fig. 8d, when the thermal smearing becomes smaller than the plateau width. For smller $T, A$ 
is controlled by the curvature on the plateau. The temperature at which $A$ has a peak, defined as $T_{A}^{*}$, is plotted as diamonds in Fig, 4 (the peak is only found for $x \leq 0$ ). Rather surprisingly, $T_{A}^{*}$ scales with the plateau width $q_{c}$, even though the dynamic exponent is $z=2$. Further, the maximum slope scales approximately as $A_{\max } \sim T_{A}^{*-1.5}$, which follows from the fact that $A \sim T^{-1.5}$ at the Cpoint.

At intermediate doping, Fig. 8b,c, $A$ is generally a scaled-down version of the behavior near the two end points, with a crossover near $\mu=-0.25 \mathrm{eV}$, where the T-dependence is weak. Also for intermediate temperatures, there can be fine structure on the plateau (e.g., solid lines in Fig. 7a) which can lead to wild swings in $A(T)$. However, at these dopings they are not relevant, since the susceptibility peaks are away from $\vec{Q}$, and this fine structure is not generally reported in Fig. 8.
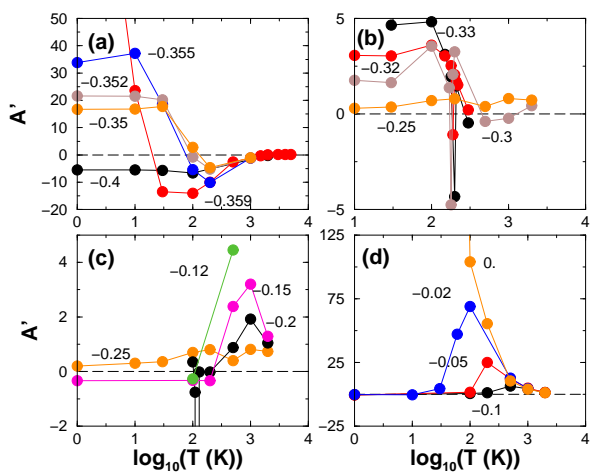

FIG. 8. Temperature dependence of $A^{\prime}$ for several dopings.

\section{Parameter Evaluation for Mode Coupling Theory}

The evaluation of the SCR parameters $A$ and $C$ was discussed above and in Appendix D. The collapse of the $\vec{q}$ and/or $\omega$ plateau widths near the $\mathrm{H}$ - and C-points leads to the introduction of additional parameters $q_{c}$ and $\alpha_{\omega}$. The narrow width of the $\vec{q}$-plateau, particularly for electron doping, leads to an additional complication not included in the conventional SCR analysis: the curvature of the bare susceptibility near $\vec{Q}=(\pi, \pi)$ (the $S$-point of the BZ) is strongly temperature dependent, and for some dopings may even change sign. In principle, it is not difficult to incorporate an $A(T)$ into the analysis near the mean-field Néel temperature $T_{N}^{*}$ (pseudogap onset). But for the present 2D system, long range Néel order only sets in at $T_{N}=0$, and for $T<<T_{N}^{*}$, a self consistent value of $A$ should be found, by taking into account the effect of the pseudogap in modifying the electronic dispersion and hence $\chi$. For the present, this complication is ignored, and in the following section $A$ is taken as $A=A\left(T_{N}^{*}\right)$, where $T_{N}^{*}$ is the magnetic pseudogap onset, the temperature where $\chi_{0}(\vec{Q}) U_{\text {eff }}=1$, using the effective $U_{\text {eff }}$ found earlier ${ }^{9}$ (Appendix B). This should be the most important $A$ for controlling the pseudogap, and moreover at lower temperatures the band renormalization should strongly modify $A(T)$. With this choice, the resulting $A(\mu)$ is plotted in Fig. 9a, along with the $C$ parameter, evaluated at $T=0$. For electron doping, this choice of $A$ is always positive and varies smoothly with doping, diverging at the C-point. By contrast, for hole doping $A$ is often negative, again illustrating the instability of the uniform AFM phase. Given $A$ and $C$, Fig. 10 shows the calculated values of $\chi_{\vec{Q}}$ and $\omega_{s f}$, normalized to $\xi^{2}$.
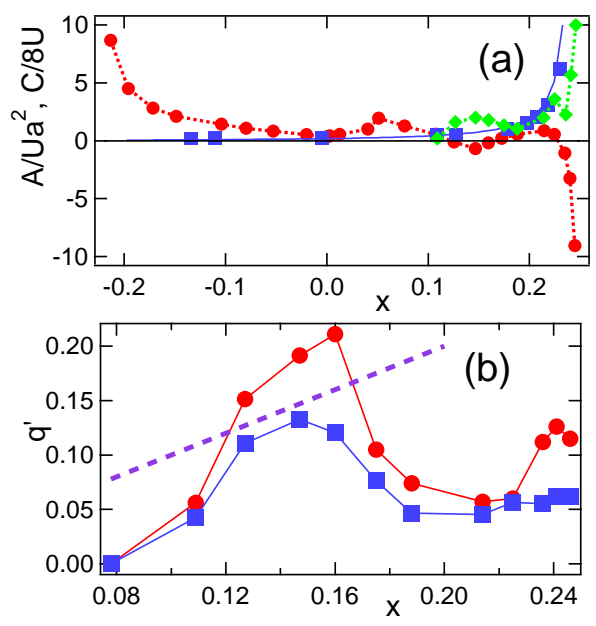

FIG. 9. (a) Calculated values of $A$ (circles for commensurate $\vec{Q}$, diamonds for incommensurate $\left.\vec{q}=\vec{Q}+\vec{q}^{\prime}\right)$ and $C$ (squares). Solid line $=$ Eq. D7. (b) Incommensurate wavevector $q^{\prime}$ in two different directions: circles along $(1,0)$, squares along $(1,1)$; dashed line: $q^{\prime} \propto x$.

For hole doping, the incommensurability creates difficulties in defining the SCR model. The mean field transition temperature at the incommensurate vector $q$ is only marginally higher than that at $Q$, Fig. 2, suggesting that incommensurability should have only a small efect on the phase diagram. Thus, one might attempt to define a positive $A$ by maasuring the curvature from an incommensurate nesting vector. However, this $A$ is highly anomalous, for a number of reasons. First, the incommensurate $\vec{q}^{\prime}$ $\left(\vec{q}=\vec{Q}+\vec{q}^{\prime}\right)$ forms roughly a square around $\vec{Q}$, insert in Fig. 4, with the peak susceptibility generally along the $(\pi, 0)$ axis (circles in Fig. 9b). In this case, by symmetry there are four peaks in the susceptibility, at $\left(\pi \pm q^{\prime}, \pi\right)$ and at $\left(\pi, \pi \pm q^{\prime}\right)$. Moreover, the curvature measured from any incommensurate peak is highly anisotropic, since the susceptibility is nearly constant along the ridge of the square, with a shallow minimum at $(\pi, \pi)$. Thus parallel to the ridge, $A_{\|}$is nearly zero. Moreover, perpendicular to the ridge, $A_{\perp}$ takes on very different values on the sides of the ridge displaced toward or away from $(\pi, \pi)$. The curvature is small, with significant deviations from quadratic (weaker curvature) moving toward $(\pi, \pi)$, while moving away from $(\pi, \pi)$, the curvature is larger, and de- 
viating toward stronger curvature as the susceptibility falls off the edge of the plateau. For reference purposes, the average value of the quadratic part of $A_{\perp}$ is plotted as diamonds in Fig. 9a. (In this case, $\omega_{s f}$ has a peak near the VHS, Fig. 10.) This definition of $A$ is almost certainly an overestimate. In the analysis of hole-doped cuprates in Sections VI and VII, the commensurate SCR model will be applied, with $A$ as a free parameter. It will be found that agreement with measurement requires a somewhat smaller value for $A$ than the estimated value of $A_{\perp}$. This small $A$ value, combined with the broad plateau, lead to a sum-rule saturation for $\chi_{0}$ and a much slower divergence of $\xi(T)$ than found for electron doped cuprates.

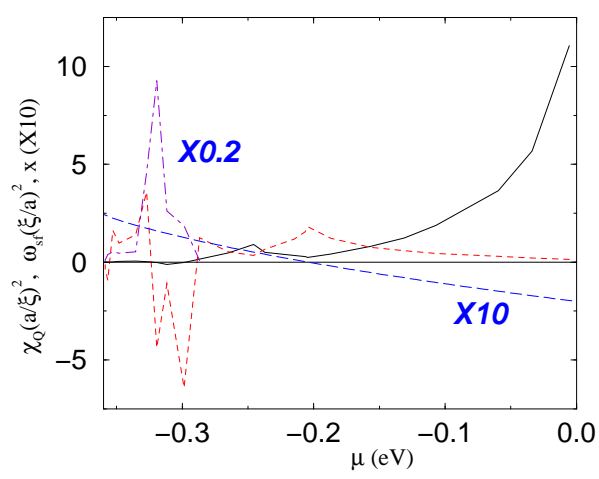

FIG. 10. Calculated values of $\chi_{\vec{Q}} / \xi^{2}$ (solid line) and $\omega_{s f} \xi^{2}$ (short dashed line), assuming $U=6 t$. Long dashed line $=$ doping $x(\mu)(\times 10)$; dot-dashed line $=\omega_{s f} \xi^{2}$ corrected for incommensurate $\vec{q} \neq \vec{Q}(\times 1 / 5)$.

In the following section, the present results are applied to understanding the ARPES spectra of electron doped cuprates, concentrating on the four dopings analyzed by Armitage, et al. ${ }^{8}$. For convenience, Table I summarizes the parameters for these dopings. From the mean-field analyses ${ }^{9}$, the effective Hubbard parameters were found to be $U_{\text {eff }} / t=6(x=0), 5(x=-0.04), 3$ $(x=-0.10)$, and $2.5(x=-0.15)$. [These numbers differ somewhat from those of Ref. 9, which included a second neighbor hopping, $t^{\prime \prime}$, to give the best fit of the Fermi surfaces.] The Stoner factor has a quantum correction, $\eta$, Eq. 10, which tends to suppress the AFM transition; hence a smaller renormalization of $U$ is required. This is reflected in Table 1: for $x=-0.1,-0.15$, there are two rows, the upper row using the mean-field $U$ parameters, the lower with the quantum correction. Note that the $U$ 's are enhanced by essentially the quantum correction factor. These values will be used in the subsequent analysis.

The SCR analysis also involves a mode coupling parameter $u$. An attempt to directly calculate $u$ (Appendix D4) failed, giving anomalously small values (Table I) due to the flatness of the susceptibility plateau $(\partial \chi / \partial \omega \sim 0)$, Fig. 39d. This problem has been noted previously, although there is debate about whether $u$ diverges $^{33}$ or vanishes ${ }^{35,36}$. Here $\rho_{s}$ is estimated from the measured correlation length for $x=0$, using Eq. 20, as discussed in Section VII. Since $\rho_{s} \propto u^{-1}$, Eq. 22, this gives $u^{-1}=0.384 \mathrm{eV}$, which is assumed for all dopings. The calculated values of $\rho_{s}$ are illustrated in Fig. 11b, based on Eqs. C1, 22.

Table I: Electron Doped Cuprates

\begin{tabular}{||c|c|c|c|c|c|c|c|c||}
\hline \hline $\mathrm{x}$ & $U / t$ & $A / a^{2}$ & $\omega_{1}(\mathrm{eV})$ & $\alpha_{\omega}$ & $q_{c} a$ & $\eta$ & $T_{A}^{*}(K)$ & $u^{-1}(\mathrm{eV})$ \\
\hline \hline 0 & 6 & 0.696 & 0.345 & 0.583 & 0.635 & 1.20 & 1020 & 760 \\
\hline-0.04 & 5 & 1.16 & 0.540 & 0.455 & 0.518 & 1.17 & 850 & 3200 \\
\hline-0.10 & 3 & 1.34 & 1.32 & 0.176 & 0.342 & 1.15 & 500 & 2700 \\
\hline$"$ & 3.5 & 1.56 & 1.13 & 0.206 & $"$ & 1.13 & $"$ & 2300 \\
\hline-0.15 & 2.5 & 1.75 & 2.16 & 0.054 & 0.172 & 1.09 & 56 & 4000 \\
\hline$"$ & 2.9 & 2.03 & 1.86 & 0.062 & $"$ & 1.05 & $"$ & 3500 \\
\hline
\end{tabular}

It is convenient to compare the present results with parameters estimated for the SCR model ${ }^{32}$ from experimental data for (optimally) hole-doped cuprates. The parameters are defined as $T_{0}=A q_{B}^{2} / 2 \pi C, T_{A}=A q_{B}^{2} / 2 \chi_{0}$, $y_{0}=\delta_{0}(T=0) / A q_{B}^{2}$, and $y_{1} \simeq 12 a^{2} u / \pi^{3} A C$. The results are listed in Table II, where the first line gives the hole-doped results estimated in Ref. 32. Moriya, et al. ${ }^{32}$ took $q_{B}^{2}=1 / 4 \pi a^{2}\left(q_{B} a=0.282\right)$, while for Table II it is assumed that $q_{B}=q_{c}$. A key difference is that Moriya, et al. ${ }^{32}$ assume the system is in the paramagnetic phase $\left(y_{0}>0\right)$ at and above optimal (hole) doping, while in the present work $y_{0}<0$, and the system is paramagnetic due to the Mermin-Wagner theorem, with the Mott gap appearing as a pseudogap. The small magnitude of $y_{0}$ is suggestive of a system pinned close to a QCP. Finally, the parameter $y_{1}$ is estimated using the value $u^{-1}=0.384 \mathrm{eV}$ (above), and not the anomalous values of Table 1 .

Table II: SCR Parameters

\begin{tabular}{||c|c|c|c|c||}
\hline \hline $\mathrm{X}$ & $T_{0}(\mathrm{~K})$ & $T_{A}(\mathrm{~K})$ & $y_{0}$ & $y_{1}$ \\
\hline \hline$\sim 0.2$ & $1600-4000$ & $3000-10000$ & $0.01-0.02$ & 3 \\
\hline 0.0 & 180 & 1150 & -5.27 & 0.75 \\
\hline-0.04 & 310 & 1300 & -3.31 & 0.7 \\
\hline-0.10 & 380 & 670 & -1.23 & 1.5 \\
\hline-0.15 & 200 & 220 & -0.31 & 1.85 \\
\hline
\end{tabular}

\section{ARPES SPECTRA}

\section{A. SCR Transition and Correlation Length}

Given the above parameters, the doping dependence of the MF and SCR transitions is compared in Fig. 11 for the four electron dopings studied in Refs. 8, 9. The MF transition occurs when the bare Stoner factor $\delta_{0}=$ $1-\chi_{\vec{Q} 0} U$ becomes negative, Fig. 11a. However, in SCR the renormalized Stoner factor $\delta$ stays positive, so there is no $T>0$ phase transition (Mermin-Wagner theorem), although $\delta-\delta_{0}$ has a strong increase near the temperature where $\delta_{0}$ changes sign. There is still a zero-T Néel transition, controlled by the quantum corrected Stoner factor, 
$\bar{\delta}_{0}=\eta-\chi_{\vec{Q} 0} U$. From Fig. 11c, it can be seen that at $x=-0.15$, the system is close to a $\mathrm{QCP}, \bar{\delta}_{0}(T=0) \rightarrow 0$. This QCP is controlled by the Stoner criterion of the zero- $\mathrm{T}$ antiferromagnet. While there is no long range order, there is still a Mott (pseudo)gap, controlled by short-range order, Fig. 11d. A direct comparison of the transition temperatures is presented on a linear $\mathrm{T}$ scale in Fig. 12. The spin stiffness $\rho_{s}$ (Fig. 11b) is found to be nearly $T$-independent below the pseudogap onset.

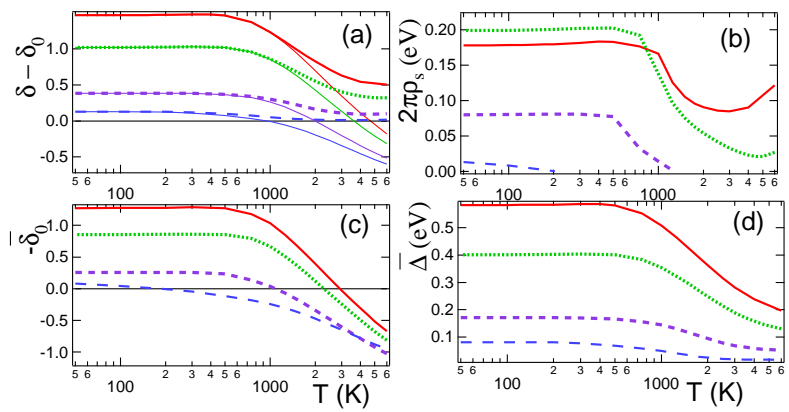

FIG. 11. (a) $\delta-\delta_{0}$ (thin solid lines $=-\delta_{0}$ ); (b) $\rho_{s}$ calculated from Eqs. 22 , 14; (c) $-\bar{\delta}_{0}$; (d) $\bar{\Delta}$, Eq. 31. In all the plots, the solid curves correspond to $x=0.0$, dotted lines: $x=-0.04$, short dashed lines: $x=-0.10$, long dashed lines: $x=-0.15$.

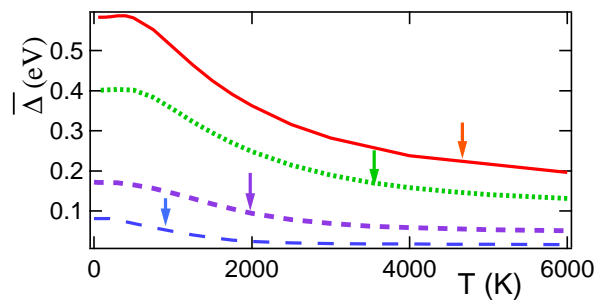

FIG. 12. Temperature dependence of gap $\bar{\Delta}$ for (from highest to lowest) $x=0,-0.04,-0.10$, and -0.15 . Arrows show mean field transition temperature $T_{N}$.

While the value of $U$ has been adjusted to fit the ARPES spectra, it is important to note that good agreement has also now been found with magnetic properties. This is discussed in Section VII.

\section{B. General Results}

Given the susceptibility, Eq. 15, the self energy can be calculated approximately as

$$
\begin{array}{r}
\Sigma\left(\vec{k}, i \omega_{n}\right)=\frac{g^{2} \chi_{0}}{\beta V} \sum_{\vec{q}, i \omega_{m}} G_{0}\left(\vec{k}+\vec{q}, i \omega_{n}+i \omega_{m}\right) D_{0}\left(\vec{q}, i \omega_{m}\right) \\
=\frac{g^{2} \chi_{0}}{V} \sum_{\vec{q}} \int_{-\alpha_{\omega} / C}^{\alpha_{\omega} / C} \frac{d \epsilon}{\pi} \frac{n(\epsilon)+f\left(\xi_{\vec{k}+\vec{q}}\right)}{i \omega_{n}+\epsilon-\xi_{\vec{k}+\vec{q}}} \frac{C \epsilon}{\left(\delta+A q^{\prime 2}\right)^{2}+(C \epsilon)^{2}},
\end{array}
$$

with bare Green's function $G_{0}\left(\vec{k}, i \omega_{n}\right)=1 /\left(i \omega_{n}-\xi_{\vec{k}}\right)$, $\xi_{\vec{k}}=\epsilon_{\vec{k}}-\mu$, and magnetic propagator $D_{0}$, Eq. 7 ; for the form of the integral, see the discussion near Eq. 9. In addition, $\chi_{0}=\chi_{0}(\vec{Q}, 0), \vec{q}=\vec{Q}+\vec{q}^{\prime}, n$ is the Bose function, and

$$
g^{2} \chi_{0}=U^{2} \chi_{0}\left(U \chi_{0}\left(\vec{Q}, i \omega_{n}\right)+\frac{1}{1+U \chi_{0}\left(\vec{Q}, i \omega_{n}\right)}\right) \simeq \frac{3 U}{2}
$$

(Ref. 53). The last form is an approximation based on the empirical substitution $\chi_{0} \rightarrow \simeq 1 / U$ in the pseudogap regime. [An improved approximation for $\Sigma,\left(G_{0} \rightarrow G\right.$ in Eq. 26) is discussed in Section V.] After analytical continuation, the imaginary part of the retarded self energy is

$$
\begin{aligned}
\operatorname{Im} \Sigma^{R}(\vec{k}, \omega)= & \frac{-g^{2} \chi_{0}}{V} \sum_{\vec{q}} \int_{-\alpha_{\omega} / C}^{\alpha_{\omega} / C} d \epsilon\left[n(\epsilon)+f\left(\xi_{\vec{k}+\vec{q}}\right)\right] \times \\
& \times \delta\left(\omega+\epsilon-\xi_{\vec{k}+\vec{q}}\right) \frac{C \epsilon}{\left(\delta+A q^{\prime 2}\right)^{2}+(C \epsilon)^{2}} .
\end{aligned}
$$

The resulting self energy is plotted in Fig. 13 for $T=$ $100 K$. (The weak oscillations seen in some branches of $\Sigma_{I}$ are an artifact due to an insufficient density of points in the numerical integration.) Note that $\operatorname{Im} \Sigma$ has the form of a broadened $\delta$-function peaked at $\omega=\xi_{\vec{k}+\vec{Q}}$. If it were a $\delta$-function, $\operatorname{Im} \Sigma=-\pi \bar{\Delta}^{2} \delta\left(\omega-\xi_{\vec{k}+\vec{Q}}\right)$, then

$$
\operatorname{Re} \Sigma^{R}(\vec{k}, \omega)=\frac{1}{\pi} \int_{-\infty}^{\infty} d \epsilon \frac{\operatorname{Im} \Sigma^{R}(\vec{k}, \epsilon)}{\epsilon-\omega}=\frac{\bar{\Delta}^{2}}{\omega-\xi_{\vec{k}+\vec{Q}}},
$$

so away from the $\delta$-function

$$
G(\vec{k}, \omega)=\frac{1}{\omega-\xi_{\vec{k}}-\operatorname{Re} \Sigma^{R}(\vec{k}, \omega)}=\frac{\omega-\xi_{\vec{k}+\vec{Q}}}{\left(\omega-\xi_{\vec{k}}\right)\left(\omega-\xi_{\vec{k}+\vec{Q}}\right)-\bar{\Delta}^{2}} .
$$

This is exactly the Green's function of the mean field calculation $^{1,54}$, with the substitution $\Delta \rightarrow \bar{\Delta}$, where $\bar{\Delta}$ can be evaluated by integrating

$$
\begin{array}{r}
\bar{\Delta}^{2}=-\frac{1}{\pi} \int_{-\infty}^{\infty} d \omega \operatorname{Im} \Sigma^{R}(\vec{k}, \omega) \\
=\frac{U}{8 u}\left(\delta-\delta_{0}\right),
\end{array}
$$

Fig. 11d. This result is due to the Bose term $n(\epsilon)$ in the square bracket of Eq. 28, the Fermi function $f$ making no contribution. This leads to $\bar{\Delta}$ being independent of $\vec{k}$. 


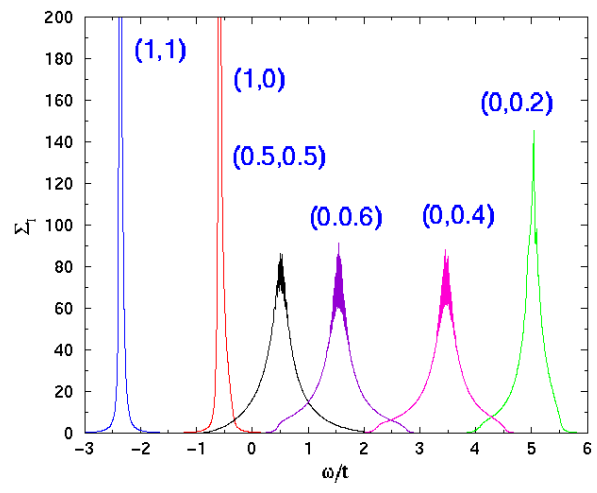

FIG. 13. Imaginary part of the self energy, Eq. 28, assuming $1 / C=0.05 t, \delta=0.002, \alpha_{\omega}=1, T=100 \mathrm{~K}$. The branches are labelled $\left(k_{x}, k_{y}\right)$, in units of $\pi$.

Equations 30,31 constitute an important result, the connection between the Mott gap and short-range magnetic order ${ }^{55,56}$. Recalling that $\Delta=U<M_{i}>$, or $\Delta^{2}=U^{2}<S_{i}>^{2}$, where $<M_{i}>=(-1)^{i}<S_{i}>$ is the staggered magnetization, then, in the spirit of an alloy analogy, a short-range order parameter can be defined as

$$
\begin{gathered}
\bar{\Delta}_{S R}^{2}(i \omega)=\frac{-g^{2}}{4 \beta} \int_{0}^{\beta} \sum_{<i, j>}<S_{i+}(\tau) S_{j-}(0)>e^{i \omega \tau} d \tau \\
=\frac{-g^{2}}{4 \beta} \sum_{k}\left(c_{x}+c_{y}\right) \chi_{+-}(k, i \omega) \simeq \frac{g^{2}}{2 \beta} \sum_{k} \chi_{+-}(k, 0)
\end{gathered}
$$

which is equivalent to Eq. 31. (In the last equality in Eq. 32 the limit $i \omega \rightarrow 0$ is an adiabatic approximation ${ }^{11}$, while the approximation is made that $\chi$ peaks near $\vec{Q}$. Thus, as long as there is short-range magnetic order $(\bar{\Delta}$ or $\rho_{s}$ non-zero), there will be a Mott (pseudo)gap.

\section{Application to the Cuprates}

Using the correct $\operatorname{Im} \Sigma^{R}$ from Eq. 28, and the calculated parameter values from Table I, ARPES spectra are calculated for electron-doped cuprates, at the four dopings for which detailed data are available ${ }^{8}$. The resulting dispersions are shown in Fig. 14. There is a well defined pseudogap, with two peaks in the spectral function at a given $\vec{k}$. It should be stressed that since there is no interlayer coupling, long range antiferromagnetic order exists only at $T=0 K$. The agreement with the mean field results $^{9}$, Fig. 15, and experiment ${ }^{8}$ is quite good, except that the SCR gap is smaller at half filling. This is due to lack of self-consistency: in calculating the self-energy, a susceptibility based on the bare Green's function was used, neglecting the opening of a gap near the Fermi level. In Section V it will be shown that when this is accounted for (via the self-consistent Born approximation) a larger gap is found. For completeness, Fig. 16 shows the mean field dispersion in the three-band model, discussed in Appendix A. The overall agreement in all cases is quite striking.
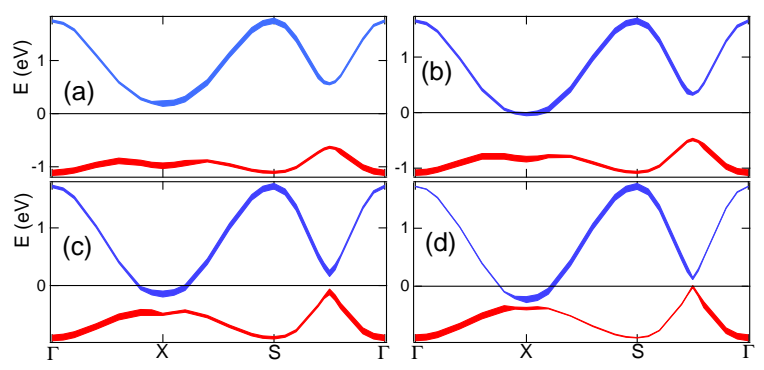

FIG. 14. SCR Dispersion relations for electron doped materials, calculated at $T=100 K$ : (a) $x=0(U / t=6)$, (b) $x=-0.04(U / t=5),(\mathrm{c}) x=-0.10(U / t=3.5)$, and $(\mathrm{d})$ $x=-0.15(U / t=2.9)$. Linewidth indicates relative intensity; for $x=-0.15$ all shadow features are extremely weak.

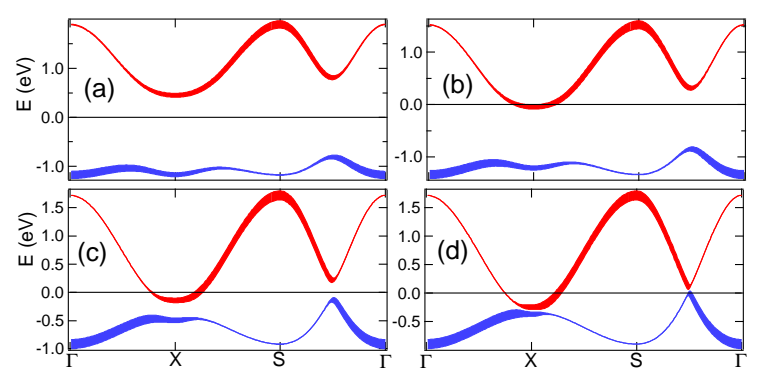

FIG. 15. Mean field dispersion relations for electron doped materials, calculated at $T=1 K$ : (a) $x=0(U / t=6)$, (b) $x=-0.04(U / t=5),(\mathrm{c}) x=-0.10(U / t=3)$, and $(\mathrm{d})$ $x=-0.15(U / t=2.6)$. Linewidth indicates relative intensity.

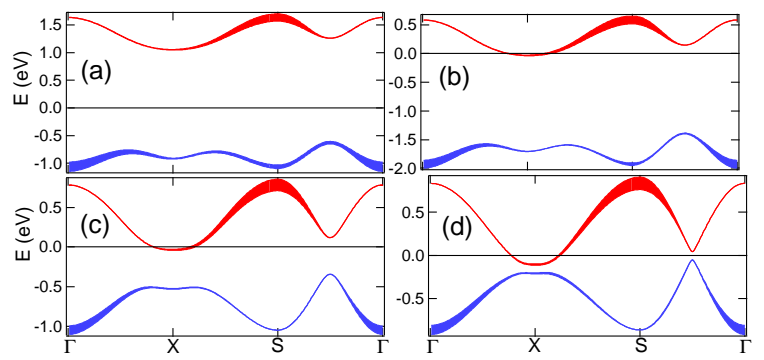

FIG. 16. Mean field dispersions in three-band model for electron doped materials, showing the two antibonding bands, assuming $m_{Q}=0.3$ (a), 0.2 (b), 0.05 (c), and 0.01 (d). Other parameters are discussed in Appendix A.

In an earlier calculation ${ }^{12}$ a somewhat larger value of $u$ was assumed, $u^{-1}=0.256 \mathrm{eV}$. This leads to stronger quantum corrections: the parameter $\eta-1$ (Table I) was about twice as large and the gaps in Fig. 14 were smaller, particularly near half filling.

Figure 17 shows typical calculated spectra for several 
$\vec{k}$-points in the a-b plane. Broadened Hubbard bands are found, which gradually smear out at high temperatures as $\delta$ increases ( $\xi$ decreases).

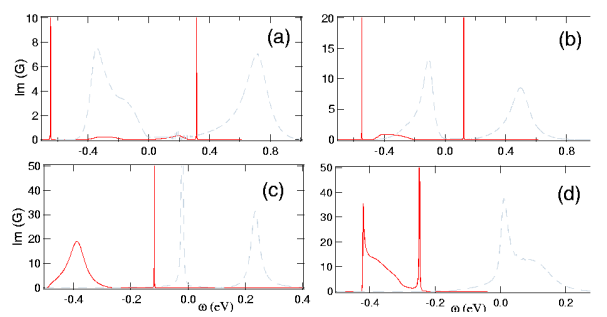

FIG. 17. Spectral functions for (a) $x=0$, (b) $x=-0.04$, (c) $x=-0.10$, and (d) $x=-0.15$, at $T=100 K$. Solid lines at $(\pi, 0)$, and long dashed lines at $(\pi / 2, \pi / 2)$.

Figures 18- 20 illustrate the temperature dependence of $\operatorname{Im}(G)$ and $\operatorname{Im}(\Sigma)$ for two dopings, $x=0$ and -0.15 . The broadening of the peaks can be understood from Eq. 28: particle-hole excitations are present within a range $\pm \alpha_{\omega} / C$ of $\xi_{\vec{k}+\vec{q}}$. Away from this particle-hole continuum the main peaks are sharp, while they broaden when they enter the continuum.
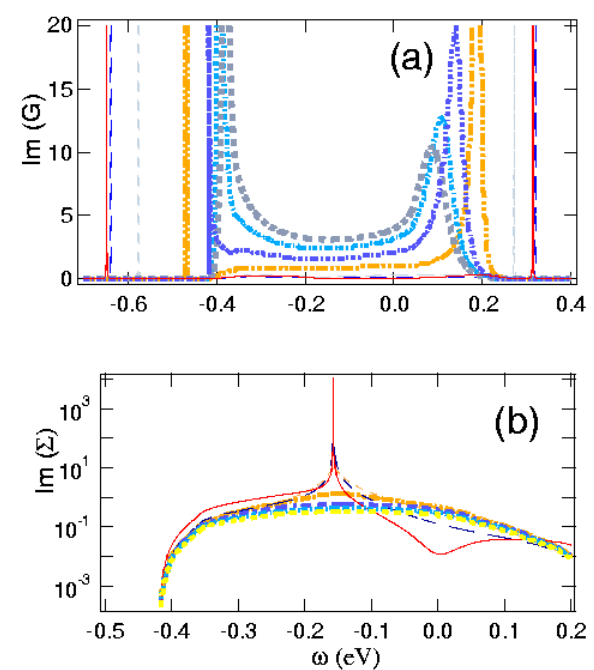

FIG. 18. Temperature dependence of (a) spectral function and (b) imaginary part of self energy, for $x=0.0$ at $(\pi, 0)$. Temperatures are 100, 500, 1000, 2000, 3000, 4000, and $5000 \mathrm{~K}$.

Note that the Mott gap collapse is anisotropic: for the undoped case, the nodal gap collapses between 2$3000 \mathrm{~K}$, while a gap persists near $(\pi, 0)$ above $5000 \mathrm{~K}$. $\operatorname{Im}(\Sigma)$ has striking oscillatory structure, particularly near $(\pi / 2, \pi / 2)$, which produces a similar weak structure in $\operatorname{Im}(G)$ at low T. [Similar, weaker oscillations are present near $(\pi, 0)$, which can be better seen in Fig. 4c of Ref. 12.] In addition, there is a very intense, strongly T-dependent peak in $\operatorname{Im}(\Sigma)$ exactly at $\xi_{\vec{k}+\vec{q}}$ (Fig. $18 \mathrm{~b}$ - also present but not shown in Fig. 19b - see Section
IX.B). It is the divergence of this peak as $T \rightarrow 0$ which signals the AFM transition. At low temperatures, the peak positions in $\operatorname{Im}(G)$ have a temperature dependence consistent with the collapse of the Mott gap - e.g., the LHB shifts to higher energies (toward midgap) at higher temperatures. Some experiments on hole doped cuprates find the opposite dependence ${ }^{57}$, which can possibly be understood as a localization or phase separation effect.
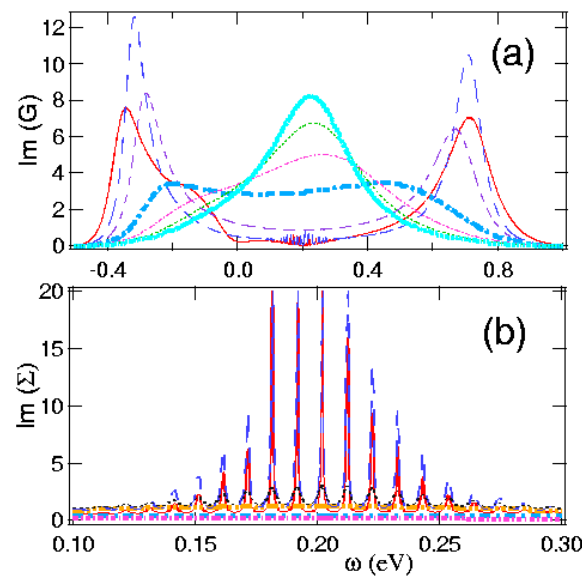

FIG. 19. Temperature dependence of (a) spectral function and (b) imaginary part of self energy, for $x=0.0$ at $(\pi / 2, \pi / 2)$. Temperatures are $100,500,1000,2000,3000$, 4000 , and $5000 \mathrm{~K}$.

In contrast, for $x=-0.15$, Fig. 19, the splittings are absent near $(\pi / 2, \pi / 2)$, and vanish near $(\pi, 0)$ by $\sim 500 \mathrm{~K}$, and the lines actually sharpen on warming. If the effective $U$ is reduced to $2.5 t$, no splitting is found, but the peak position and broadening have an anomalous $\mathrm{T}$ dependence. Clearly, the system is very close to a QCP. Figure 21 shows in more detail how the spectrum evolves with $U$ near this point.

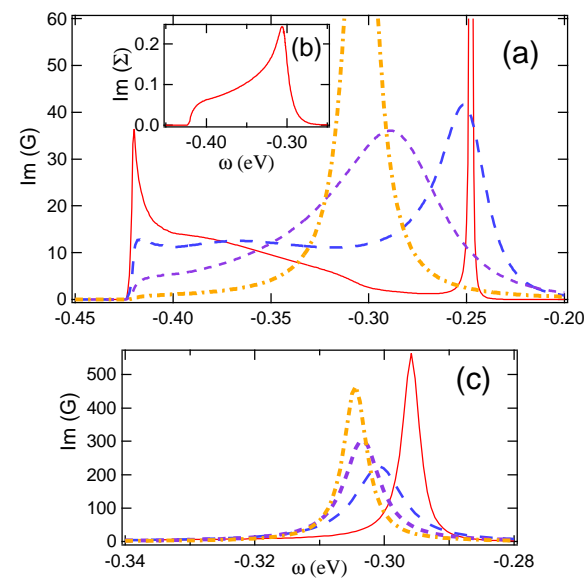


FIG. 20. Temperature dependence of spectral function for $x=-0.15$ at $(\pi, 0)$, for $U / t=2.9$ (a) and 2.5 (c). Temperatures are 100 (solid line), 500 (long-dashed line), 1000 (short-dashed line), and 2000K dot-dashed line). (b): imaginary part of self energy at $T=100 \mathrm{~K}, U / t=2.9$.

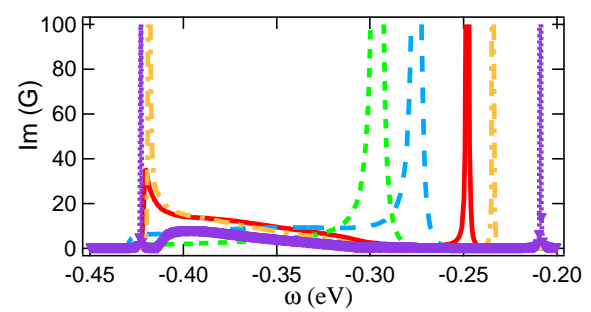

FIG. 21. $U$-dependence of spectral functions for $x=-0.15$ at $T=100 K$ near the $T=0 \mathrm{QCP}$, for $U / t=2.5$ (short dashed line), 2.7 (long dashed line), 2.9 (solid line), 3.0 (dot-dashed line), and 3.2 (dotted line).

Finally, Fig. 22 displays Fermi surface maps for $x=$ -0.10 and -0.15 , showing the crossover from small to large Fermi surface. Hot spot effects are prominent at $x=-0.15$, pinning the Fermi surface to the zone diagonal and broadening it at a pseudogap due to hot-spot scattering ${ }^{58}$. These should be compared with the meanfield ${ }^{9}$ and experimental ${ }^{8}$ results. It should be noted that in the mean field calculation, it was necessary to include a $t^{\prime \prime}$ parameter to reproduce the experimental hole pocket near the zone diagonal. Such a parameter would have shifted the Fermi surface across the zone diagonal, leading to improved agreement with experiment here as well.

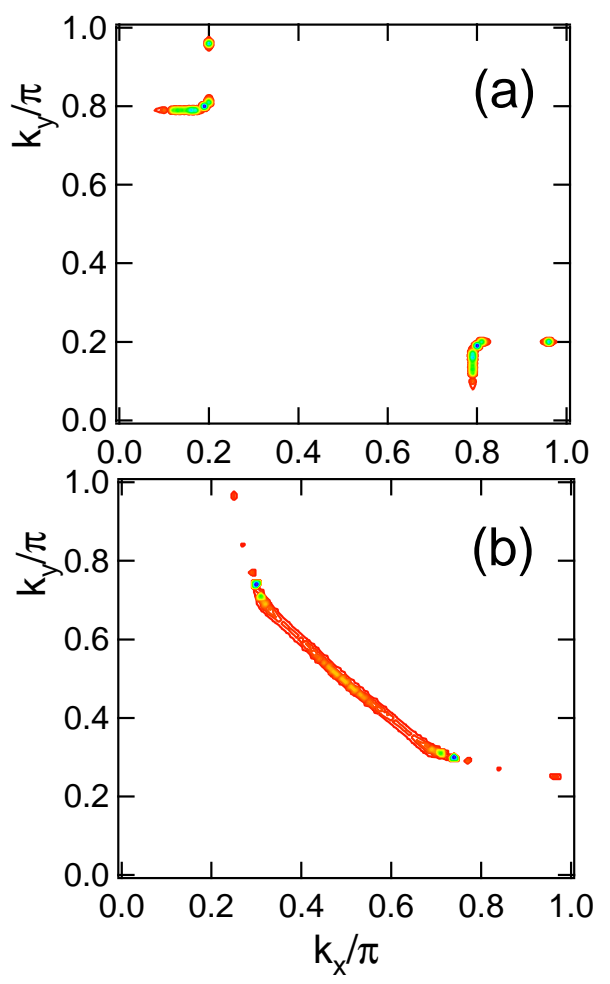

FIG. 22. Fermi surface map for $x=-0.10$ (a) and -0.15 (b).

Thus, the SCR calculation agrees with the mean-field results $^{9}$, if the mean-field gaps and transition temperatures are interpreted as the opening of a pseudogap at finite $\mathrm{T}$, with the long-range AFM appearing only at $\mathrm{T}=0$. Moreover, the overall dispersions, Fig. 14 are in quite good agreement with the mean field results ${ }^{9}$ and experiments $^{8}$.

\section{SELF-CONSISTENT BORN APPROXIMATION}

A limitation of the above calculations is that the self energy Eq. 26 is calculated using the bare susceptibility, whereas the full susceptibility should be strongly modified by the opening of the Mott gap. This can be corrected for by including the full Green's function into the self energy calculation. This is conveniently done at $T=0$, since there is a long-range ordered phase, and the sole difference between the SCR and mean-field calculations is a weak renormalization of the Hubbard $U$. In this case, a renormalized Green's function can be found by summing all the non-crossing diagrams; this is the self-consistent Born approximation (SCBA). By including the interaction of the quasiparticles with spin waves, it also incorporates the physics of magnetic polarons. Magnetic polarons closely resemble lattice polarons, leading to both coherent and incoherent contributions to the spectral function, with considerable bandwidth renormalization in the coherent spectrum. In the $t-J$ model it is known that the SCBA gives a good description of exact diagonalization results on small lattices ${ }^{59}$.

Here, a simple calculation is presented to estimate the effect of the SCBA corrections on the dispersion of the insulating phase. Only the coherent band dispersion is included, and the SCBA is applied to the RPA solution ${ }^{60}$, which should be similar to the ordered SCR phase at $T=$ 0 . The calculation of Chubukov and Morr ${ }^{55}$ is extended to include both lower and upper Hubbard bands. The RPA dispersions of the upper (c) and lower (v) Hubbard bands can be written as follows. If the bare dispersion is $\epsilon_{k}=-2 t\left(\cos \left(k_{x} a\right)+\cos \left(k_{y} a\right)\right)-4 t^{\prime} \cos \left(k_{x} a\right) \cos \left(k_{y} a\right)-$ $2 t^{\prime \prime}\left(\cos \left(2 k_{x} a\right)+\cos \left(2 k_{y} a\right)\right)$, then defining $\epsilon_{k}^{( \pm)}=\left(\epsilon_{k} \pm\right.$ $\left.\epsilon_{k+Q}\right) / 2, E_{k}^{(-)}=\sqrt{\epsilon_{k}^{(-) 2}+\Delta^{2}}, \Delta=U<S_{z}>$, then

$$
E_{k}^{c, v}=\epsilon_{k}^{(+)} \pm E_{k}^{(-)} \text {. }
$$

Here $\Delta$ is the AFM gap, $\Delta \sim U / 2$ at half filling. For large $\Delta$, this can be expanded as

$$
\begin{aligned}
E_{\vec{k}}^{c, v}= & A_{00}+A_{01} \cos k_{x} a \cos k_{y} a+ \\
& +A_{02}\left(\cos 2 k_{x} a+\cos 2 k_{y} a\right),
\end{aligned}
$$

with 


$$
\begin{gathered}
A_{01}=J / 2 \pm t^{\prime}, \\
A_{02}=J / 2 \pm 2 t^{\prime \prime} .
\end{gathered}
$$

The same dispersion is found in the $t-J$ model $^{61,62}$, suggesting that the SCBA will be an equally good approximation here.

The self-consistent equation (replacing Eq. 26) can be written

$$
\begin{array}{r}
G^{-1}(k, \omega)=\omega-\left(E_{k}^{c, v}-\mu\right) \\
-\int \frac{d^{2} q}{4 \pi^{2}} \Psi_{c, v}(k, q) G\left(k+q, \omega+\omega_{q}\right)
\end{array}
$$

where $\Psi_{c, v}$ is a vertex correction for the upper (c) or lower (v) Hubbard band and $\omega_{q}$ is the spin wave dispersion. As will be seen below (Eq. 48), $\Psi \propto t^{2}$, so Eq. 37 is independent of $t$, depending only on ratios $t^{\prime} / t, t^{\prime \prime} / t$, and $J / t$. However, the final dispersion also scales with $t$, so any comparison with experiment requires all four parameters. For an arbitrary electronic dispersion, these quantities can be evaluated as follows. The transverse susceptibility in the RPA can be written as ${ }^{1,63}$

$$
\bar{\chi}^{+-}(\mathbf{q}, \mathbf{q}, \omega)=
$$

$\frac{\chi_{0}^{+-}(\mathbf{q}, \omega)\left[1-U \chi_{0}^{+-}(\mathbf{q}+\mathbf{Q}, \omega)\right]+U\left[\chi_{Q}^{+-}(\mathbf{q}, \omega)\right]^{2}}{\left[1-U \chi_{0}^{+-}(\mathbf{q}, \omega)\right]\left[1-U \chi_{0}^{+-}(\mathbf{q}+\mathbf{Q}, \omega]-U^{2}\left[\chi_{Q}^{+-}(\mathbf{q}, \omega)\right]^{2}\right.}$,

$\frac{\chi_{Q}^{+-}(\mathbf{q}, \omega)}{\left[1-U \chi_{0}^{+-}(\mathbf{q}, \omega)\right]\left[1-U \chi_{0}^{+-}(\mathbf{q}+\mathbf{Q}, \omega]-U^{2}\left[\chi_{Q}^{+-}(\mathbf{q}, \omega)\right]^{2}\right.}$,

$$
\bar{\chi}^{+-}(\mathbf{q}, \mathbf{q}+\mathbf{Q}, \omega)=
$$

with

$$
\begin{gathered}
\chi_{0}^{+-}(\mathbf{q}, \omega)=\frac{1}{2 N} \sum_{k}^{\prime}\left[1-\frac{\epsilon_{k}^{(-)} \epsilon_{k+q}^{(-)}-\Delta^{2}}{E_{k}^{(-)} E_{k+q}^{(-)}}\right] \times \\
\times\left[\frac{1}{E_{k}+E_{k+q}-\omega}+\frac{1}{E_{k}+E_{k+q}+\omega}\right],
\end{gathered}
$$

and

$$
\begin{gathered}
\chi_{Q}^{+-}(\mathbf{q}, \omega)=\frac{1}{2 N} \sum_{k}^{\prime} \frac{\Delta\left(E_{k}^{(-)}+E_{k+q}^{(-)}\right)}{E_{k}^{(-)} E_{k+q}^{(-)}} \times \\
\quad \times\left[\frac{1}{E_{k}+E_{k+q}-\omega}-\frac{1}{E_{k}+E_{k+q}+\omega}\right] .
\end{gathered}
$$

In the large $U$ limit, Eq. 38 becomes

$$
\bar{\chi}_{0}^{+-}(\mathbf{q}, \mathbf{q}, \omega)=\eta_{q}^{2}\left[\frac{1}{\omega+\omega_{q}}-\frac{1}{\omega-\omega_{q}}\right],
$$

with

$$
\eta_{q}^{2}=\frac{1}{2} \sqrt{\frac{a_{q}-\gamma_{q}}{a_{q}+\gamma_{q}}}
$$

$$
\begin{gathered}
\omega_{q}=2 J \sqrt{a_{q}^{2}-\gamma_{q}^{2}}, \\
\gamma_{q}=\frac{\cos \left(q_{x} a\right)+\cos \left(q_{y} a\right)}{2},
\end{gathered}
$$

and

$$
a_{q}=1+\frac{J^{\prime}}{J}\left(1-\cos \left(q_{x} a\right) \cos \left(q_{y} a\right)\right)+\frac{J^{\prime \prime}}{J}\left(1-\gamma_{2 q}\right),
$$

with $J=4 t^{2} / U, J^{\prime} / J=\left(t^{\prime} / t\right)^{2}, J^{\prime \prime} / J=\left(t^{\prime \prime} / t\right)^{2}$. As befits a Goldstone mode, $\omega_{q}=0$ at $q=(0,0)$ and $(\pi, \pi)$. In this case, $\Psi_{c, v}=\Phi_{c, v}^{2}$, with

$$
\Phi_{c, v}=\bar{\eta}_{q}\left(\epsilon_{k}^{(-)}-\epsilon_{k+q}^{(-)}\right) \pm \eta_{q}\left(\epsilon_{k}^{(-)}+\epsilon_{k+q}^{(-)}\right),
$$

with $\bar{\eta}_{q}=1 /\left(2 \eta_{q}\right)$, or

$$
\Psi_{c, v}=16 t^{2}\left[\frac{a_{q}\left(\gamma_{k}^{2}+\gamma_{k+q}^{2}\right)-2 \gamma_{k} \gamma_{k+q} \gamma_{q}}{\sqrt{a_{q}^{2}-\gamma_{q}^{2}}} \pm\left(\gamma_{k}^{2}-\gamma_{k+q}^{2}\right)\right] \text {. }
$$

Given $\omega_{q}, E_{k}^{c, v}$, and $\Psi_{c, v}$, Eq. 37 can be solved numerically to find both the coherent and incoherent parts of the ARPES spectral weight. However, the incoherent (38art contributes to a weak background, and the experimental spectra are generally compared to the coherent part. Hence, for present purposes what is needed is the dispersion of the coherent part of $G$. Following Chubukov and Morr ${ }^{55}$ this can be simplified. The Green's function has the form

$$
G(k, \omega)=\frac{Z}{\omega-\omega_{\max }+\bar{E}_{k}-i \gamma\left(\omega-\omega_{\max }\right)^{2} \Theta\left(\omega_{\max }-\omega\right)},
$$

with quasiparticle residue $Z$, band edge $\omega_{\max }$, damping $\gamma$, dispersion $E_{k}$, with step function $\Theta(x)=1(0)$ for $x$ $>(<) 0$. The quasiparticle residue can be found as

$$
\frac{1-Z}{Z^{2}}=\int \frac{d^{2} q}{4 \pi^{2}} \frac{\Psi\left(k_{0}, q\right)}{\left(\omega_{q}+E_{k_{0}+q}\right)^{2}},
$$

where $k_{0}$ is the band-edge momentum: $\vec{k}_{0}=(\pi / 2, \pi / 2)$ $[(\pi, 0)]$ for hole [electron] doping. (With the conventional signs $t^{\prime}<0, t^{\prime \prime}>0$; in the special case $t^{\prime}=t^{\prime \prime}=0$, both energies are degenerate.) An equation for the dispersion can then be found by substituting Eq. 49 into Eq. 37, and setting $\omega=\omega_{\max }$ :

$$
\begin{gathered}
\bar{E}_{k}^{c, v}=Z E_{k}^{c, v}-Z^{2} e_{k}^{c, v} \\
\left.e_{k}^{c, v}=\int \frac{d^{2} q}{4 \pi^{2}}\left[\frac{\Psi(k, q)}{\omega_{q}+E_{k+q}}-\frac{\Psi\left(k_{0}, q\right)}{\omega_{q}+E_{k_{0}+q}}\right]\right]
\end{gathered}
$$

(The damping adds a small correction to the dispersion, which we ignore.) It is convenient to rewrite Eq. 34 as 


$$
\begin{aligned}
& E_{k}^{c, v}=4 A_{01}\left(c_{01}+\cos \left(k_{x} a\right) \cos \left(k_{y} a\right)\right) \\
& +A_{02}\left(c_{02}+\cos \left(2 k_{x} a\right)+\cos \left(2 k_{y} a\right)\right),
\end{aligned}
$$

with $c_{01}=0(1), c_{02}=2(-2)$ for the lower (upper) Hubbard band. It is found that $\bar{E}_{k}$ satisfies a similar equation, with renormalized $A_{0 i} \rightarrow A_{i}$. In this case, the selfconsistent equation Eq. 51 can be reduced to a pair of equations at fixed $k$-values. For example, at $k=(0,0)$

$$
\begin{array}{r}
4\left(1+c_{01}\right) A_{1}+\left(2+c_{02}\right) A_{2} \\
=Z\left[4\left(1+c_{01}\right) A_{01}+\left(2+c_{02}\right) A_{02}\right]-Z^{2} e_{(0,0)}^{c, v},
\end{array}
$$

with a similar equation at $\vec{k}=(\pi, 0)$ [or $(\pi / 2, \pi / 2)]$. Figure 23 illustrates the self-consistent values of $Z, A_{1}$, and $A_{2}$ as a function of $J$ for fixed $t^{\prime}, t^{\prime \prime}$.
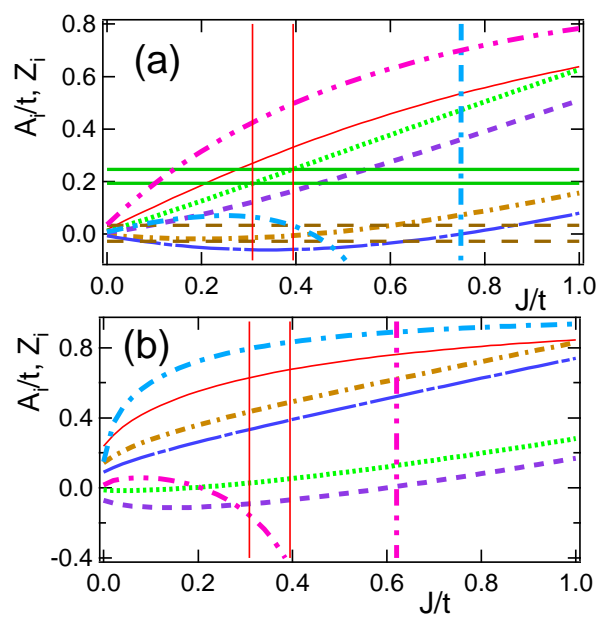

FIG. 23. Renormalized parameters for lower (a) and upper (b) Hubbard bands: $Z$ (solid lines), $A_{1}$ (dot-dashed lines), and $A_{2}$ (dotted lines), in comparison with $Z A_{01}$ (long-dashed-short-dashed lines), and $Z A_{02}$ (short-dashed lines), assuming parameters $t=0.326 \mathrm{eV}, t^{\prime}=-0.375 t$, and $t^{\prime \prime}=0.15 t$. Horizontal lines $=$ experimental range for $A_{1}$ (long dashed lines) and $A_{2}$ (solid lines), after Refs. [ 64, 65]. Also shown are the individual renormalization factors $Z_{1}$ (long-dash-dotted line) and $Z_{2}$ (long-dash-dot-dotted line). Vertical lines delimit parameter values consistent with experiment.

Note that any attempt to extract the bare parameters from the measured dispersion is highly underdetermined. Thus, while the band dispersion $\bar{E}_{k}$ and spin wave dispersion $\omega_{q}$ depend explicitly on $J, t^{\prime}$, and $t^{\prime \prime}$, the vertex function depends on $t$, so there are four parameters to determine, but only two parameters $A_{1}$ and $A_{2}$ can be found from the ARPES dispersion. Moreover, from Fig. 23a, the value $A_{1}$ is insensitive to $J$ in the range of interest. In principle, the parameters can be determined from additional measurements, including the Mott gap $\Delta$, the spin wave velocity $c_{s}\left(\right.$ as $\left.q \rightarrow 0, \omega_{q} \rightarrow c_{s} q\right)$,

$$
c_{s}=2 a \sqrt{J\left(\frac{J}{2}+J^{\prime}+2 J^{\prime \prime}\right)},
$$

or the maxima in the spin wave spectra, $\omega_{(\pi / 2, \pi / 2)}=$ $2\left(J+J^{\prime}+2 J^{\prime \prime}\right), \omega_{(\pi, 0)}=2\left(J+2 J^{\prime}\right)$.

Given this indeterminancy, a simplified picture is assumed here to estimate parameter changes: the renormalized value of $t=0.326 \mathrm{eV}$ is assumed fixed, to keep $\Psi$ and the experimental ratios $A_{i} / t$ constant, and further, the ratio $t^{\prime} / t^{\prime \prime}=-2.5$ was assumed constant. Then the pairs of solid and long-dashed horizontal lines in Fig. 23a give the experimental ranges ${ }^{64,65}$ for $A_{1}$ and $A_{2}$ respectively. A reasonable match can be found for a bare $t^{\prime}=-0.375 t$. In this case, the value of $A_{2}$ suggests a bare $J$ in the range $0.33-0.41 t$, or $108-135 \mathrm{meV}$. For the same parameter range, the individual parameters are renormalized by $Z_{i}=A_{i} / A_{0 i}$, with $Z_{1} \simeq 0.059-0.020[0.8$ $0.84], Z_{2} \simeq 0.44-0.51$ [-0.21 - -0.54] for the lower [upper] Hubbard band. The ratio $j / t^{\prime \prime}$ must be renormalized by the SCBA, since $A_{2}$ and $A_{02}$ cross zero at different values of $J$, causing $Z_{2}$ to be negative for the upper Hubbard band (it diverges when $A_{02} \rightarrow 0$ ).
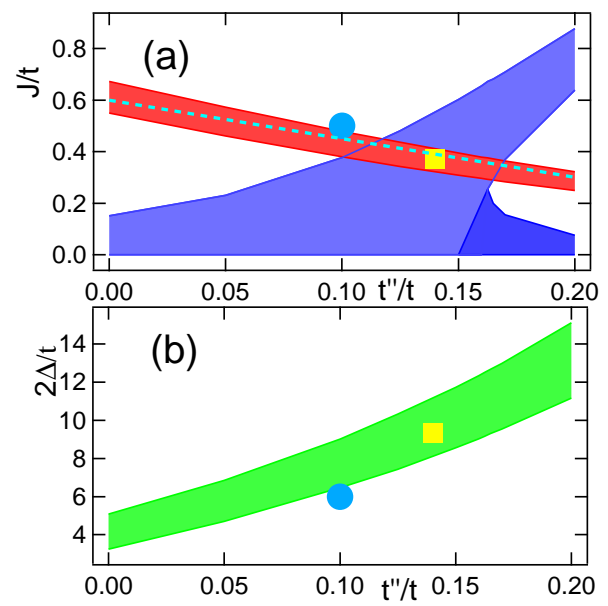

FIG. 24. Parameter values consistent with ARPES data for $J$ (a) and $2 \Delta$ (b). Broad range determined by $A_{1}$; narrow range (in both a and b) by $A_{2}$. Circle $=\mathrm{SCR}$ result, square $=$ best SCBA approximation. Dashed line in a: $J=0.6 t-1.5 t^{\prime \prime}$.

The above calculation can be repeated for different values of $t^{\prime \prime}$, and the allowed parameter values for $J$ and $t^{\prime \prime}$ are shown in Fig. 24a. The 'best' SCBA value (square) differs from the SCR value (circle) by less than a factor of two. Since $J$ is reduced by polaron coupling, $U=4 t^{2} / J$ must increase, Fig. 24b. This can be seen directly from the self-consistent equation for $G$. The leading edge of the band is found from $\operatorname{Re}\left(G^{-1}\left(k_{0}, \omega_{\max }\right)\right)=0$, or

$$
\omega_{\max }=\omega_{\max 0}+\int \frac{d^{2} q}{4 \pi^{2}} \frac{\Psi\left(k_{0}, q\right)}{\omega_{q}+E_{k_{0}+q}} .
$$

The gap $2 \Delta$ is equal to the splitting between the upper and lower Hubbard bands at $(\pi / 2, \pi / 2)$ - it is not the sum of the $\omega_{\max }$ 's for these two bands, since the bottom of the upper Hubbard band lies at $(\pi, 0)$. Correcting for the renormalization of the dispersion at $(\pi / 2, \pi / 2)$ reduces 
the gap, but even so the renormalized $\Delta$ (Fig. 25, shortdashed line) is larger than the bare value (solid line). Figure 26 shows that the SCBA increases the Mott gap near half filling, which corrects a shortcoming of the SCR model, noted above.

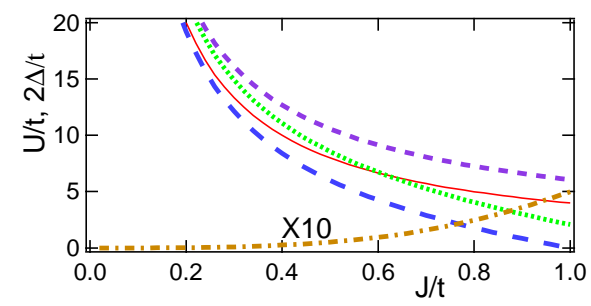

FIG. 25. Renormalized $U$ parameter as a function of $J$ : solid line $=$ bare $U=4 t^{2} / J$ in large gap limit; short-dashed line $=$ renormalized $U$ from Eq. 56; long-dashed line $=$ bare $\Delta$ corrected for the small gap limit, Eq. 56; dotted line $=$ renormalized $U$ in the small gap limit from Eq. 56; dot-dashed line $=A_{03}$.

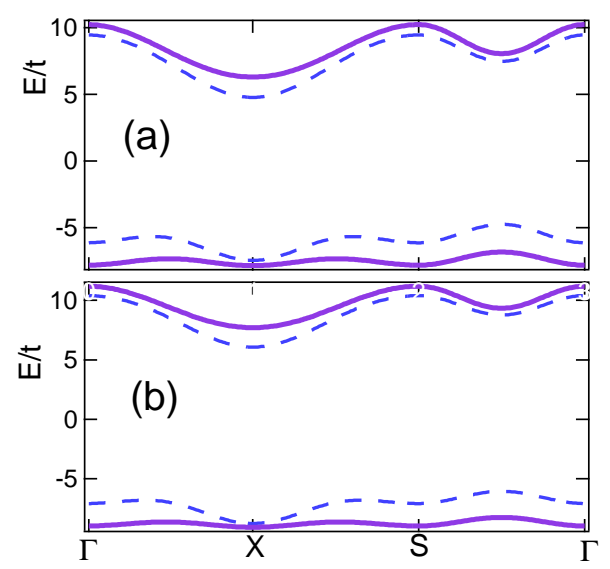

FIG. 26. Comparison of mean-field (dashed lines) and SCBA (solid lines) dispersions for $t^{\prime}=-0.375 t, t^{\prime \prime}=0.15 t$, and two choices of $J, J / t=0.42$ (a) or 0.33 (b).

\section{A. Extension to Small $U$}

The above results were valid for the large- $U$ limit, where the gap parameter $\Delta=U<S_{z i}>\rightarrow U / 2>>t$, in which case $J=2 t^{2} / \Delta$. As $\Delta$ decreases, certain modifications are necessary. The most important is a modification of $J$. From the above analysis, the susceptibility, spin wave dispersion, and renormalized band parameters all depended on the bare electronic dispersion. Hence, the value of $J$ should be chosen to best approximate the bare $A_{0 i}$, Eqs. 35,36 . This can be accomplished by matching the exact dispersion to the approximate form at $\vec{k}=(0,0)$, or

$$
\frac{J}{t}=\frac{\Delta}{4 t}\left[\sqrt{1+\left(\frac{4 t}{\Delta}\right)^{2}}-1\right],
$$

Fig. 27. Note that $J \rightarrow 1$ as $\Delta \rightarrow 0$, Fig. 25. It is interesting to note that when the renormalization correction, Eq. 56, is added in, the renormalized $\Delta$ (dotted line in Fig. 25) lies close to the perturbative result $\Delta=2 t^{2} / J$ (solid line).
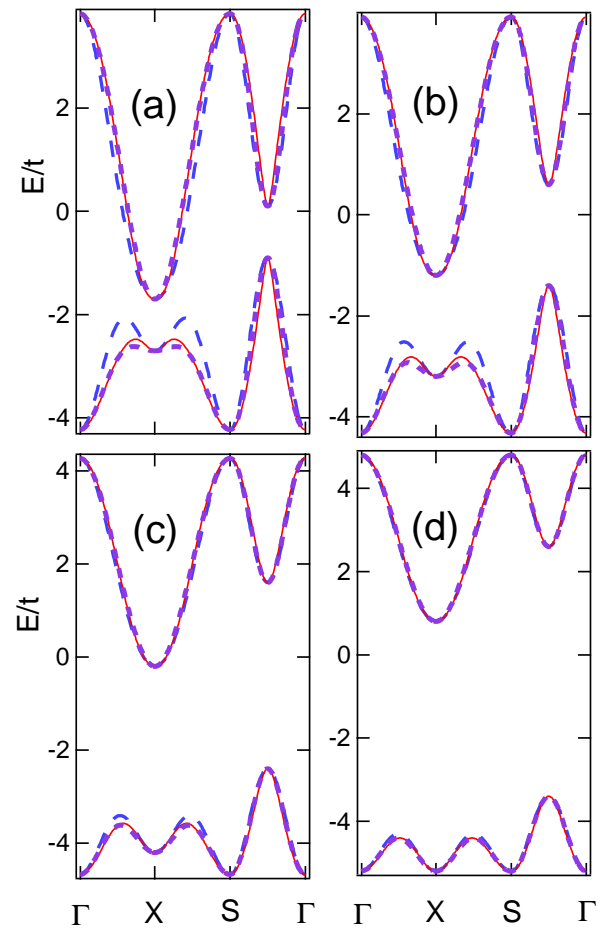

FIG. 27. Mean field band structure (solid lines) plus approximations involving Eqs. 57, 58, with (short-dashed lines) or without (long-dashed lines) a finite $A_{03}$, for $\Delta / t=0.5$ (a), 1.0 (b), 2.0 (c), and 3.0 (d).

For small $\Delta$ an additional correction is required, to account for quartic corrections in $t / \Delta$. This can be done, as above, by adding a term $A_{03}\left(1-c_{2 x} c_{2 y}\right)$ to the model bare dispersion, which allows a fit to the exact bare dispersion at $(\pi / 2,0)$, if

$$
\frac{A_{03}}{t}=\frac{\Delta}{2 t}\left[\sqrt{1+\left(\frac{2 t}{\Delta}\right)^{2}}-1\right]-\frac{J}{2 t} .
$$

This yields a very good approximation to the dispersion down to $\Delta=t / 2$, Fig. 27 (short dashed line). The parameter $A_{03}$ is plotted in Fig. 25 .

\section{B. Summary of SCBA Results}

(1) Thus at half filling polaronic effects renormalize the bandwidth by only a factor of $\sim 2$, with some change in lineshape. Polaronic effects reduce the values of $J, t^{\prime}$, and $t^{\prime \prime}$, and hence increase the value of $U$. Thus the gap is enhanced at half filling, correcting a shortcoming of the SCR calculation (Figs. 14, 15). 
While the present calculations are restricted to half filling, some additional features can be extracted from the calculations of Kusunose and Rice $(\mathrm{KR})^{13}$. (2) While considerable weight is transferred to an incoherent spectrum, the coherent spectrum is quite similar to that found in RPA and SCR calculations, and it is this component which is mainly seen in the ARPES spectra. Possible evidence for the incoherent states is a second peak seen in ARPES spectra of half-filled cuprates ${ }^{66}$, about $0.6 \mathrm{eV}$ below the main peak of the LHB near the nodal point. While KR find an incoherent peak at half filling about $2.5 t$ below the first peak, its intensity actually maximizes away from the nodal direction toward $\Gamma$, while the experimental peak is stronger in the opposite direction, towards $(\pi, \pi)$.

(3) An important result of the RPA and SCR calculations is that $U$ must decrease with doping to reproduce the experimentally observed crossover to a large Fermi surface. The same result has been found by Sénéchal and Tremblay ${ }^{14}$. The results of KR are consistent, in that KR kept $U$ doping independent, and did not find this crossover.

(4) Whereas in the mean-field and SCR calculations, electron doping shifts the Fermi level into the UHB without affecting the relative weights of the two subbands, in the SCBA the UHB states below the Fermi level are formed by spectral weight transfer from the LHB. This spectral weight transfer had been seen experimentally, and its absence was known to be a shortcoming of mean field theory, which is thus seen to be corrected in the SCBA.

(4) In lightly doped NCCO, Armitage, et al. ${ }^{8}$ found an additional weak pseudogap - actually a leading edge gap at the Fermi level of the UHB - which was not reproduced by the SCR calculation. Such a pseudogap is found by KR, and in an earlier calculation by Stanescu and Phillips ${ }^{67}$. KR interpreted this as evidence that the filled states were not actually part of the UHB but were in-gap states close to the bottom of the UHB. Similar in-gap states had been proposed for hole-doped cuprates $^{68}$, and have been considered as evidence for stripes. [In LSCO, where stripes are most clearly observed, the added states are close to mid-gap ${ }^{69}$; in other hole-doped cuprates, the evidence is less clear, but if in-gap states exist, they must lie close to the top of the LHB.]

The connection between polarons and stripes is a delicate issue: for very light doping one would expect magnetic polarons to form for both hole and electron doping. These polarons are strongly dressed electrons, with many features of second-phase inclusions, and have been suggested to act as precursors for nanoscale phase separation ${ }^{70,71}$. In hole-doped cuprates, there is considerable evidence that these polarons tend to cluster and form stripes. In electron-doped cuprates there is considerably less evidence for stripes, and it may be that polarons do not form clusters. Hence, the differences between a polaronic phase and a stripe phase might be rather subtle.

\section{EXTENSION TO HOLE DOPED CUPRATES}

Thus, for electron-doped cuprates, a three-fold coincidence of Mott gap collapse, Fermi surface crossover, and zero-T QCP is found. SCR theory predicts a similar triad for the hole doped cuprates, and the present section explores the extent to which this is found experimentally.

\section{A. Pseudogap}

In hole doped cuprates, ARPES finds two features which are commonly referred to as pseudogaps - a 'hump' feature found near $(\pi, 0)$ at higher binding energy than the main, superconducting 'peak', and the 'leading edge gap', a loss of spectral weight in the immediate vicinity of the Fermi level. This latter feature is not explained by the present calculation; it may be the magnetic feature discussed in Section V.B ${ }^{67,13,14}$, or it may be associated with the onset of strong superconducting fluctuations ${ }^{56,72}$.

On the other hand, the 'hump' feature can be consistently interpreted as the collapse of the Mott pseudogap ${ }^{56}$. Bilayer splitting cannot explain SIN tunneling measurements ${ }^{72,73,44}$ which find two hump-like features, roughly symmetric about the Fermi level. Correlation with ARPES suggests that the tunneling peaks reflect structure near $(\pi, 0)$, and Figure 28 shows that semi-quantitative agreement with experiment can be attained in terms of weakly split Hubbard bands, for a screened $U=2.3 t$ (see also Fig. 2b). For simplicity, the calculation is carried out at the mean-field level. Figure $28 \mathrm{c}$,d shows how the bottom of the UHB near $(\pi, 0)$ gradually merges into the VHS of the LHB. The intensities and positions of the two dos peaks reveal a clear asymmetry. As the Mott gap vanishes, the two peaks merge into the VHS of the bare band. (There may be complications due to nanoscale phase segregation, since STM studies suggest that the peak and hump features are spatially segregated ${ }^{74}$.)
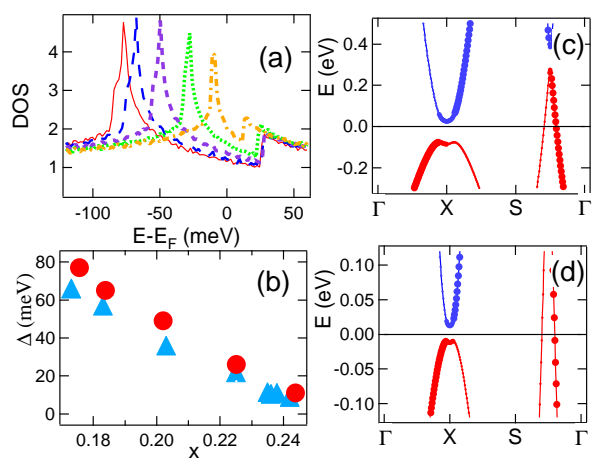
FIG. 28. (a) Calculated dos for a series of hole doped cuprates, assuming $U_{\text {eff }}=2.3 t$, with $x=0.176$ (solid line), 0.184 (long dashed line), 0.202 (short dashed line), 0.225 (dotted line), and 0.244 (dot-dashed line). (b) Comparison of shift of lower dos peak (circles) from (a) with representative tunneling data [ 44] (triangles). (c,d) Band dispersion near the pseudogap for $x=0.176$ (c) and $0.244(\mathrm{~d})$.

The above interpretation requires that for hole doping also the Mott gap must collapse slightly above optimal doping. This is consistent with recent experimental observations of a $\mathrm{QCP}^{75}$. Moreover, the model predicts that at the QCP, where the pseudogap just closes, the Fermi level is exactly at the VHS (H-point). This result had been found experimentally in some lightly overdoped cuprates $^{76,77}$.

In a recent confirmation of the QCP just beyond optimal hole doping ${ }^{78}$, it is suggested that this 'has to do with the restoration of the Fermi-liquid state in the overdoped regime characterized by a large Fermi surface' (emphasis added); a similar conclusion was made by Balakirev, et al. ${ }^{79}$. Thus two elements of the QCP triad are present. The third is more elusive.

\section{B. $\mathrm{T}=0 \mathrm{QCP}$}

In electron-doped cuprates, a finite Néel temperature persists all the way to the QCP; by contrast, for hole doping $T_{N} \rightarrow 0$ at a doping $x 0.02-0.03$, considerably below the proposed QCP. Here it is suggested that a $\mathrm{T}=0$ magnetic transition persists out to the QCP, but the correlation length grows so slowly that three-dimensional Néel order is superceded by the superconducting transition. Details are presented in a related publication ${ }^{80}$, and only briefly summarized here.

The key insight is that the susceptibility must satisfy the fluctuation-dissipation theorem ${ }^{11,26}$

$$
<M^{2}>=-\int \frac{d \omega}{\pi} n(\omega) \int \frac{d^{2} q}{(2 \pi)^{2}}\left(\frac{c_{x}+c_{y}}{2}\right) \operatorname{Im} \chi(\vec{q}, \omega)
$$

where $\left\langle M^{2}>\right.$ is the mean square local amplitude of nearest neighbor spin fluctuations and $n$ is the Bose function. For hole-doped cuprates the $q$-plateaus constitute a problem. For electron-doped cuprates, the plateau width is quite small, and the susceptibility is large only over an area $\xi^{-2}$, so the sum rule Eq. 59 is never saturated, and $\chi_{Q}$ and $\xi$ both diverge exponentially with decreasing $\mathrm{T}$. For hole doping the plateau width is large, Fig. 4, and the curvature on the plateau $A q^{2}$ is relatively small, so as $\mathrm{T}$ decreases intensity grows all across the plateau. This tends to saturate the sum rule, leading to a greatly weakened divergence of the correlation length,

$$
\xi^{2}=\frac{a}{T}-b
$$

with $a$ and $b$ constants. From Eqs. 6, 9, it can be shown that

$$
\begin{array}{r}
a \simeq \frac{A \pi\left|\delta_{0}\right|}{3 u a^{2} q_{c}^{2}} \\
\simeq \frac{8 \pi^{2} A<M^{2}>}{U \chi_{0 Q} q_{c}^{2}},
\end{array}
$$

(where the latter form follows from Eq. 59, and $\chi_{0 Q}=$ $\left.\chi_{0}(\vec{Q}, 0)\right)$ so $a \rightarrow 0$ at the QCP.

This result has a number of consequences: (1) neutron diffraction ${ }^{48,49}$ measures the plateau width, Fig. 4 and not the correlation length. (2) $\mathrm{NMR}^{81}$ measures the correlation length, and in YBCO finds a weak $T \rightarrow 0$ divergence of $\xi$, as predicted. Thus the present results resolve a longstanding ${ }^{82,81}$ controversy about the correlation length in hole-doped cuprates. (3) In the cuprates, Néel order appears at $T>0$ only if the correlation length exceeds $100 a$, where $a$ is the lattice constant ${ }^{83}$ (the connection between $T_{N}$ and $\xi$ is discussed in the next section). This explains the broad range of hole dopings where there is only $\mathrm{T}=0$ AFM order: Fig. 4 shows that the measured $\xi \rightarrow 100 a$ only at $x=0.02$ in LSCO (see also Fig. 30, below). (4) Moreover, the slope of the $T^{-1 / 2}$-term in $\xi$ decreases rapidly with doping, signalling a QCP just above optimal doping. Hence, the triad of features of the AFM QCP are also present in the hole-doped cuprates, with the broad susceptibility plateaus responsible for the striking differences from electron doping.

\section{Incommensurate Magnetism and Competing Phases}

The above analysis strongly suggests that at high energy scales the physics of the cuprates is dominated by magnetic ordering. This includes the large pseudogap regime and the attendant QCPs. None of this analysis precludes interesting new physics on lower energy scales, including of course superconductivity near the QCPs. Another possibility is the admixture of a second phase generating an enhanced gap - a popular choice being the flux phase ${ }^{84}$.

The physics associated with nanoscale phase separation, or 'stripe' physics, seems to also fall in this category. Incommensurate magnetic modulations are seen in several cuprates - particularly the LSCO family - and while the SCR model does find an incommensurate susceptibility particularly for hole doping (Fig. 9b) it probably cannot reproduce the observed doping dependence of the incommensuration. Indeed, it has been noted ${ }^{6}$ that the incommensurability generally signals an instability toward phase separation. Experiments suggest that phase separation and/or stripe physics is present in the hole doped cuprates ${ }^{85,74}$ down to arbitrarily small dopings ${ }^{86}$. However, the temperature at which stripes are stabilized seems too low ${ }^{85,87}$ for them to be directly responsible for the pseudogap phenomena. 
A detailed discussion of this issue here is clearly out of the question, but the following suggests a possible explanation. Doped carriers in an AFM are strongly dressed by their environment, forming magnetic polarons ${ }^{70}$ in a pure Hubbard model, but in a more general situation being sensitive to nearby competing phases ${ }^{88}$. Thus, it is suggested that the physics of competing phases enters the problem at the level of the properties of polarons, and different degrees of phase separation and/or stripe formation in different cuprates have to do with the tendency of polarons to cluster. That is, the stripe physics should enter the problem on a lower energy/temperature scale than the fundamental pseudogap phenomena discussed in the present paper. The $q$-plateau in hole-doped cuprates greatly enhances the sensitivity to stripe physics, since the system is close to instability over a wide range of incommensurate modulations.

\section{MAGNETIC PROPERTIES}

\section{A. Electron Doping}

While the present model was developed on the basis of ARPES data, the collapse of the Mott gap should be clearly reflected in other properties as well, in particular in the magnetic response.

Indeed, Mang, et al. ${ }^{83}$ have recently measured the ordered moment $M$ in reduced NCCO samples, and find good agreement with the present model $^{9}$ (see Fig. 30b below). The correlation length has not yet been measured in reduced (superconducting) NCCO, but there are data for the as-grown material, which is insulating ${ }^{89,83}$, Fig. 29. The reasons for the striking differences between the two types of sample are not fully understood, but there seems to be some interstitial oxygen which localizes a fraction of the doped electrons, so one must dope the as-grown samples more to produce a given reduction of the magnetic properties (e.g., to get a certain value of $T_{N}$, the doping of the as-grown sample $x_{g}$ must be about 0.02-0.03 larger than for the reduced sample $x_{r}$, inset in Fig. 29). The data for the undoped sample were used to estimate $\rho_{s}(x=0, T=0)$, and thereby $u^{-1}=0.384 \mathrm{eV}$. Comparing this to the $\sigma$-model calculations $^{29,30}, \rho_{s}=J S^{2}$, gives $J=113 \mathrm{meV}$, in good agreement with other estimates.

However, a fit to Eq. 20 could only be made by reducing the ( $T$-dependent) prefactor $\xi_{0}$ by a factor of 16 . A similar problem was encountered in the $\sigma$-model calculations: one-loop renormalization ${ }^{30}$ found $\xi_{0} \sim 1 / \sqrt{T}$, as here (below Eq. 20), while a two-loop calculation ${ }^{31}$ found a $T$-independent $\xi_{0}$. Introducing a Castro Neto-Hone-like interpolation formula ${ }^{90}$,

$$
\xi_{0}=\frac{e}{4} \sqrt{\frac{e A}{2 C\left(T+2 \pi \rho_{s}\right)}},
$$

yields the solid-line fit in Fig. 29, with no adjustment of the prefactor. Moreover, the curves for the doped samples apply the same correction factors. The agreement in T-dependence is quite good; while the theoretical $x_{r}$ is smaller than the experimental $x_{g}$, the ratio is consistent with both those derived from $T_{N}$ and from the magnetization $M$, inset in Fig. 29). This strongly suggests that as far as magnetic properties are concerned as-grown NCCO behaves like reduced NCCO, with a few percent of the electrons localized (however, as-grown NCCO never becomes superconducting).

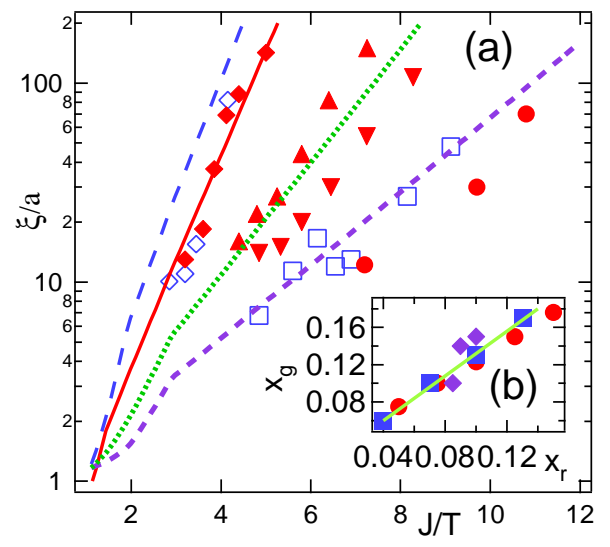

FIG. 29. Temperature dependence of correlation length $\xi$ in NCCO for $x=0$ (solid line), -0.04 (long-dashed line), - 0.085 (dotted line), and -0.10 (short-dashed line). Data are from Ref. [ 89]: $x=0$ (open diamonds) and -0.15 (open squares); from Ref. [ 83]: $x=0$ (solid diamonds), -0.10 (solid up triangles), -0.14 (solid down triangles), and -0.18 (solid circles). Fits are to Eq. 62, with parameters appropriate to $x=0$ (solid line), -0.04 (long-dashed line), -0.085 (dotted line), and -0.10 (short-dashed line). Temperatures are measured in units of $J=125 \mathrm{meV}$. Inset: Plot of as-grown nominal doping $x_{g}$ vs reduced nominal doping $x_{r}$ for fixed values of $T_{N}$ (circles), $M$ (squares), and $\xi$ (diamonds). Solid line is $x_{g}=1.2 x_{r}+0.012$.

In discussing the as-grown NCCO samples, mention should be made of the 'anomalous pseudogap'91 found in an as-grown sample near $x=-0.15$ - which should correspond to $x \sim-0.12$ in the reduced samples. From Fig. 11c, $\rho_{s}$ falls off in the range $200-1000 \mathrm{~K}$ as $x$ varies from -0.15 to -0.10 , signalling the opening of the Mott (pseudo)gap. In the as-grown sample, a pseudogap was found to open below $240 \mathrm{~K}$, centered at $300 \mathrm{meV}$. From Figs. 14, 15, the gap near $(\pi, 0)$ would be in this range. Additional infrared and Raman phonons were observed, beyond those allowed by tetragonal symmetry. This could be associated with the orthorhombic symmetry of the magnetic Brillouin zone. Clearly more work needs to be done, but if this is the correct interpretation, the present model predicts what the doping dependence should be, and that similar features should be seen in the reduced samples as well. 


\section{B. Hole Doping}

The results on NCCO should be contrasted to those for $\mathrm{LSCO}^{50}$, Fig. 30a, where a saturation of the effective $\xi$ is observed in all doped samples. For undoped $\mathrm{La}_{2} \mathrm{CuO}_{4}$, the data (open circles) largely overlap those of $\mathrm{Nd}_{2} \mathrm{CuO}_{4}$ (open and filled diamonds), but a small change of slope may be present in the best fits. For lightly-doped $\mathrm{LSCO}^{50}$, the data can be fit to Eq. 60 down to $\sim 150 \mathrm{~K}$, Fig. 30a, below which $\xi$ saturates or decreases. In principle, it should be possible to calculate this saturation of $\xi$ directly from Eq. 20. As noted in Section III.C, the value of $A$ tends to be overestimated when the susceptibility peak is incommensurate. Thus, the dotted line in Fig. 30a is the calculated value of $\xi$, using Eq. 62 with parameters appropriate to $x=0.10$ hole doping, except that $A / a^{2}=0.24$, only $1 / 3$ the value estimated from Fig. 9a.

From the $a$ coefficient of Eq. 60 it should be possible to extract the magnetization, Eq. 61. However, as explained in Section VI, neutron scattering data tend to measure the susceptibility plateau width $q_{c}$, strongly underestimating $\xi$. This is illustrated in Fig. 30b, where magnetization $M=\sqrt{\left\langle M^{2}\right\rangle}$ derived from $\xi$ via Eq. 61 is compared to $M$ in NCCO estimated from the ARPES data $^{9}$ (squares) and from magnetization (upright ${ }^{92}$ and inverted $^{83}$ triangles). The $\xi$-derived data include the NCCO neutron data of Fig. 30a (triangles) and NMR data from $\mathrm{YBCO}^{81}$ (circles) expected ${ }^{80}$ to give a better estimate of $\xi$. For both sets of data, the parameter $A / \chi_{0 Q} U a^{2}$ was taken as a constant, 2.8. Except for the lowest doping, the neutron data lead to an underestimate for $M$, confirming that the measured $\xi$ is too small. In contrast, the NMR data are consistent with the electron-doped results, and strongly suggest the presence of a QCP just above optimal doping.
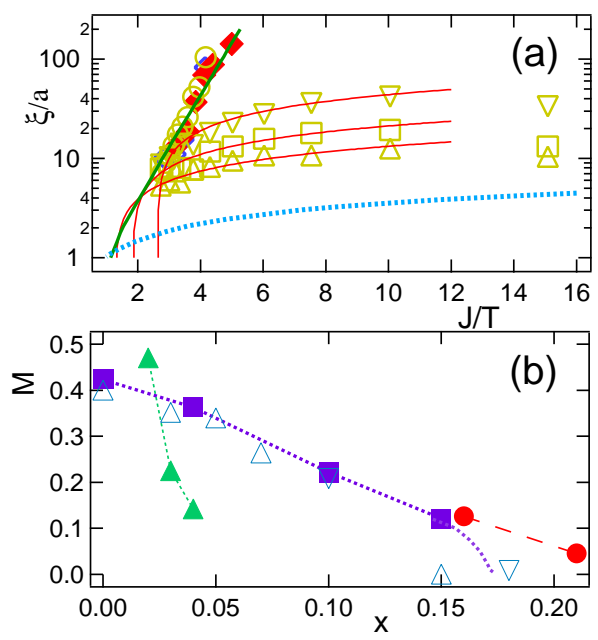

FIG. 30. (a) Temperature dependence of correlation length $\xi$ in LSCO for $x=0$ (open circles), 0.02 (inverted triangles), 0.03 (squares), and 0.04 (triangles) [from Ref. [ 50]], compared with $\mathrm{Nd}_{2} \mathrm{CuO}_{4}$ (open and filled diamonds, as in Fig. 29). Thick solid curve $=$ fit for undoped material from Fig. 29). Thin solid lines $=$ fits to Eq. 60. Dotted line $=$ calculated value for $x=0.10$, as described in the text. (b) Comparison of magnetization extracted from $\xi(T)$, Eq. 61 (triangles for $\mathrm{LSCO}^{50}$, circles for $\mathrm{YBCO}^{81,80}$ ), with that for NCCO, taken from ARPES fit, Ref. [9] (squares), and from magnetization (scaled to $M=0.4$ at $\mathrm{x}=0$; triangles: Ref. [ 92], inverted triangles: Ref. [ 83]. All lines are simply drawn to connect the data points, except for that part of the dotted line connected with the ARPES data (squares) extrapolated beyond $x=0.15$. This represents a mean-field calculation, assuming that $U$ does not change with doping over this range, and using the band parameters of Ref. [9].

More recent experiments on very lightly doped $\mathrm{LSCO}^{93}$ have found that the magnetization at these dopings is actually incommensurate - consistent with diagonal stripes. This points out an interesting parallel with the present model: early experimental samples displayed flat, diamond-shaped susceptibility plateaus near $(\pi, \pi)$. As sample quality improves, incommensurate structure seems to become more prominent: see, e.g., Fig. 1 of Ref. 94. Related behavior arises in the model: The susceptibility for hole-doped cuprates displays a flat-topped plateau at high temperatures, $\delta>0$. As the temperature is lowered, $\delta \rightarrow 0$, incommensurate structures develop from fine structure on top of the plateau, Fig. 3 of Ref. 80, gradually dominating the spectrum. However, in the calculations this incommensurability is sensitive to sample 'quality': it only shows up when $\delta$ is very close to zero. Hence, in real samples, the appearence of such structure should be very sensitive to disorder or sample inhomogeneity. Finally, it should be noted that interpretation of the incommensurability in terms of stripes remains controversial in cuprates other than the LSCO family. Reznik, et al..$^{95}$ report an approximately uniform ring of incommensurability in optimally doped YBCO, which is dispersive and pushed up to finite frequencies by the spin gap in the superconducting state. A ring or diamond of incommensurability is actually quite close to what is found here, and the extension of the present calculations to the superconducting phase should be quite similar to the results of Eschrig and Norman ${ }^{96}$.

\section{THREE DIMENSIONAL NÉEL ORDER}

The (inverse) Stoner factor $\delta_{q}$, Eq. 4 , can be generalized to include interlayer coupling:

$$
\delta_{q}(\omega)=\delta+A q^{2}+A_{z} q_{z}^{2}-B \omega^{2}-i C \omega,
$$

leading to a susceptibility 


$$
\chi(\vec{q}, \omega)=\frac{\chi_{Q}}{1+\xi^{2}\left[(\vec{q}-\vec{Q})^{2}+a_{z}\left(q_{z}-Q_{z}\right)^{2}\right]-\omega^{2} / \Delta^{2}-i \omega / \omega_{s f}},
$$

$$
J_{z}\left[\frac{\xi\left(T_{N}\right)}{\xi_{0}\left(T_{N}\right)}\right]^{2}=\Gamma T_{N}
$$

with $a_{z}=A_{z} / A$.

In the physical cuprates, the interlayer hopping has an anomalous dispersion, generally written as $t_{z}=t_{z 0}\left(c_{x}-\right.$ $\left.c_{y}\right)^{2}$. This formula holds for bilayer splitting, and in general when the $\mathrm{CuO}_{2}$ planes are stacked uniformly. However, as explained in Appendix E, many of the cuprates, including NCCO, have a staggered layering, with the $\mathrm{Cu}$ in one $\mathrm{CuO}_{2}$ plane laying above a vacancy in the neighboring $\mathrm{CuO}_{2}$ sheet. This leads to a magnetic frustration: the $\mathrm{Cu}$ in one sheet has four nearest neighbors in the adjacent sheet, two with spin up, two with spin down. This frustration is reflected in a more complicated dispersion of $t_{z}$ :

$$
t_{z}=t_{z 0}\left(c_{x}-c_{y}\right)^{2} \cos \frac{k_{x} a}{2} \cos \frac{k_{y} a}{2},
$$

which vanishes at $(\pi, 0)$ and $(0, \pi)$, and leads to a greatly reduced interlayer coupling. (Effects of AFM frustration associated with layering have been discussed in Ref. 97.)

The consequences of both uniform and staggered stacking are explored in Appendix E. If the c-axis resistivity is coherent, it can be used to estimate the interlayer hopping $t_{z 0}$. It is found that the value of $t_{z 0}$ needed to produce a given resistivity anisotropy is approximately 5 times smaller for uniform stacking, to account for the frustration in the staggered stacking. With the corresponding $t_{z 0}$ 's determined from resistivity, both forms of interlayer coupling give rise to comparable interlayer coupling, and hence a finite Néel temperature. While the optimal $Q$-vector depends on doping, at half filling both forms predict $\vec{Q}=(\pi, \pi, 0)$, consistent with experiment in $\mathrm{La}_{2} \mathrm{CuO}_{4}$. Even for quite strong anisotropy, this mechanism can account for the observed $T_{N} \mathrm{~s}$ (in fact, tends to overestimate $T_{N}$ ), without the necessity of invoking additional mechanisms, such as a Kosterlitz-Thouless transition, with the reduced spin dimensionality caused by spin-orbit coupling effects ${ }^{98-101}$.

Within mode coupling theory ${ }^{102}$ (Appendix E), the Néel temperature is found from the gap equation (Eqs. E1, C7)

$$
\chi_{0}(T) U=\eta+\frac{3 u T a^{2} \ln \left(\frac{T}{T_{3 D}}\right)}{\pi A},
$$

where $T_{3 D} \sim t_{z}^{2}$ is defined below Eq. E8. It is found that $T_{3 D}$ is approximately constant, independent of doping in the electron-doped regime. Apart from a small numerical factor, Eq. 66 differs from the isotropic three-dimensional result by the logarithmic factor, which diverges $\left(T_{N} \rightarrow 0\right)$ as $t_{z} \rightarrow 0$.

Equation 66 can be rewritten in a suggestive form. Approximating $\rho_{s}$ by $\rho_{s}^{a}=A\left(\chi_{0} U-\eta\right) / 12 u a^{2}$ (Eq. 22), then, using Eq. 20, the Néel transition occurs when where $J_{z}=J\left(t_{z 0} / t\right)^{2}, J=4 t^{2} / U$, and $\Gamma=4 t_{z 0}^{2} / U T_{3 D}$. A very similar form was proposed earlier ${ }^{103}$, and experimentally $^{83}$ Néel order seems to appear when $\xi \simeq$ $100 a$.

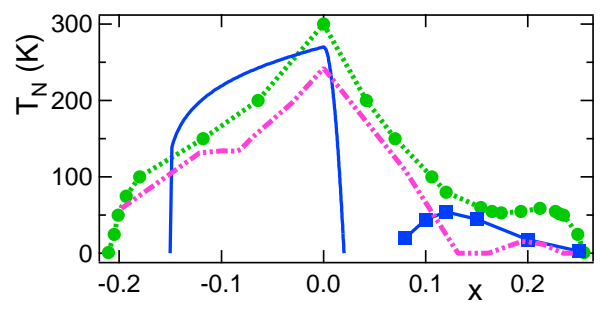

FIG. 31. Comparison of experimental Néel temperatures for NCCO and LSCO, (solid line), and for the stripe (magnetic) ordering transitions observed in Nd-substituted LSCO [ 46] (solid line with squares) with the model of interlayer coupling with staggered stacking and $t_{z 0}=t / 10 \sim 30 \mathrm{meV}$, plotted as $T_{N} / 10$ (dot-dot-dash line). Also included is the approximate expression, Eq. 68 (dotted line with circles). (Note that there is a range of hole doping for which $A$ is found to be negative; in this range $T_{N}$ was arbitrarily assumed to vanish in the staggered model, $T_{N}=0$.)

Figure 31 compares the calculated value of $T_{N}$ with the experimental values. While the overall doping dependence is comparable, the calculated $T_{N}$ is about an order of magnitude higher. The calculation is for staggered stacking, with $t_{z}$ adjusted to reproduce the observed resistivity anisotropy, but Appendix E shows that the overestimate is generic: the coefficient of the logarithm needs to be larger to reduce $T_{N}$. Also shown in Fig. 31 (dotted line) is a simplified model, which assumes that

$$
T_{0}^{*}=\frac{\pi A}{3 u a^{2} \ln \left(\frac{T}{T_{3 D}}\right)}
$$

is doping independent, $T_{0}^{*}=1200 \mathrm{~K}$. This model reproduces qualitatively the shape of the numerical calculation, but with a magnitude comparable to experiment. The magnitude of $T_{N}$ could be matched almost quantitatively if $U_{\text {eff }}$ also has a significant temperature dependence, as discussed in Appendix E . The overall doping dependence is also comparable to experiment. The agreement could be further improved by using a smaller value of $t^{\prime}$, which would shrink the doping range over which Néel order occurs.

Finally, it should be noted that a finite $T_{N}$ can change a continuous QCP into a first order. This follows beacuse the plateau width increases with increasing temperature. Hence, near the plateau edge, the system can satisfy the Stoner criterion at some finite temperature, but fail to satisfy it at a lower temperature, having fallen off of the plateau edge. Such a first order termination of the AFM state seems to be found in the electron doped cuprates, 
most notably in $\mathrm{Pr}_{2-x} \mathrm{Ce}_{x} \mathrm{CuO}_{4}(\mathrm{PCCO})^{104}$, and in a related organic material ${ }^{105}$.

\section{DISCUSSION}

\section{A. Slater vs Mott Physics}

Theories of magnetism fall into two diametrically opposed classes ${ }^{106}$ : band vs atomic models, or Slater vs Mott physics. In both approaches, the copper band is split by a Mott gap into two parts. In Slater theory, long range magnetic order leads to a unit cell doubling, so each subband remains conventional, with two electrons per unit cell. In Mott theory, the bands are highly unconventional: the gap opening is purely a local effect there is an energy penalty of $U$ for two electrons to sit on the same copper site. Since there is no change in lattice symmetry, the unit cell remains the same, and the bands hold only half as many electrons as conventional bands. Distinguishing between the two models is complicated, since in the Hubbard model, residual hopping proportional to $4 t^{2} / U \sim J$ leads to AFM coupling of the electrons, and can lead to parasitic Néel order, at a temperature $T_{N}$ much lower than that at which the Mott gap opens. On the other hand, strong fluctuations in the Slater model can greatly reduce $T_{N}$, leaving a pseudogap near the mean-field instability temperature.

While the mean field results might give a good qualitative picture of the Slater regime, they are unlikely to be able to describe Mott physics. However, it would still be hoped that the mean field results can give an indication of when the crossover is likely to occur. Here, two separate indicators are presented.

A first indication comes from looking at competing orders. A Stoner criterion $U \chi_{q}=1$ gives the onset temperature for magnetic order at $\vec{q}$, ranging from AFM, $\vec{q}=\vec{Q}$ to ferromagnetic, $\vec{q}=0$, Fig. 32 . While at half filling for any value of $U$, AFM order dominates, the splitting decreases with increasing $U$. The local, or Mott physics should arise when fluctuations to all magnetic orders are comparably likely, or the spread in transition temperatures, $\Delta T_{c}$, is $<<T$. Since the probability of a fluctuation of $N$ particles into a phase with excess free energy $\Delta f$ is $\sim e^{-N \Delta f / k_{B} T}$, one can crudely state that a phase will be significantly excited if $T_{N}-T_{c}(\vec{Q}) \leq \alpha_{0} T_{N}$, where $\alpha_{0}$ is a small numerical constant. The width of the $\Delta T_{c}$ curve in Fig. 32a shows the fraction of the Brillouin zone that is significantly excited for $\alpha_{0}=0.01$ (e.g., for $U / t \geq 32.5$, all modes are excited). This suggests that for $U \geq 15 t$, these fluctuations spread over a significant fraction of the Brillouin zone, while for $U>30 t$ virtually all magnetic states are equally excited and the Slater picture is badly broken down. However, the cuprates are generally found to be in the regime $U \leq 12 t$, where a Slater picture should be reasonably accurate even close to the $T_{N}^{*}$ crossover.

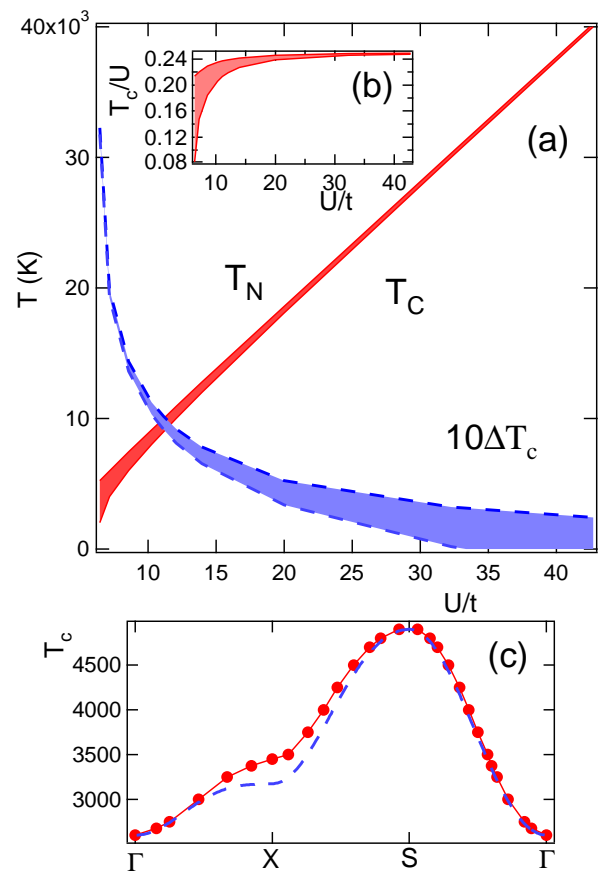

FIG. 32. (a) Mean field transition temperatures for Néel $\left(T_{N}\right)$ and ferromagnetic $\left(T_{C}\right)$ orders and their difference $\Delta T_{c}=T_{N}-T_{C}$ (upper dashed line). At any $U / t$ the ratio of the shaded area to the total area below this line gives the fraction of the Brillouin zone which is significantly excited $\left(T_{c}(\vec{Q}) \geq\left(1-\alpha_{0}\right) T_{N}\right.$, for $\left.\alpha_{0}=0.01\right)$. (b) Replot of transition temperatures scaled to $U$. (c) Plot of $T_{c}$ vs $\vec{q}$, for $U=6 t$. The curve bears an uncanny resemblance to the (scaled) electronic dispersion of the LHB, long-dashed line.
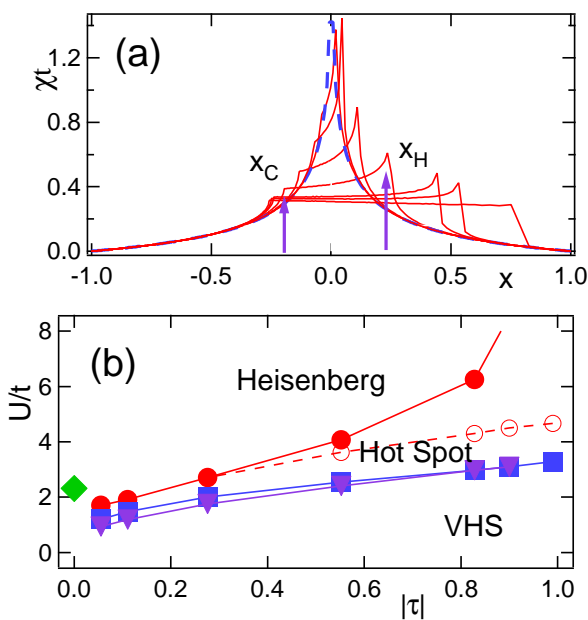

FIG. 33. (a) Bare susceptibility $\chi_{0}(\vec{Q}, 0)$ at $T=1 K$, for several values of $\tau=-0.0552,-0.110,-0.276,-0.552,-0.828$, -.9, -.99 (solid lines), and 0 (dashed line). (b) Crossover couplings as a function of $\tau$ : $U_{V}$ (triangles), $U_{2}$ (squares), and $U_{1}$ (circles). (Dashed line $=U_{1}$ for electron doping.)

Alternatively, when the mean-field solution becomes 
insensitive to the band structure, it is likely that a local picture is becoming dominant. In the present instance, the band structure is determined by the ratio $t^{\prime} / t$. For any non-zero value of $t^{\prime}$, the susceptibility has a generic doping dependence ${ }^{107,40}$, Fig. 33 - changing the sign of $t^{\prime}$ merely interchanges electron and hole doping. The role of the susceptibility plateaus can be quantified, by defining ranges of $U$ where the nature of the transition changes, Fig. 33b. Thus, for $U<U_{V}$, there is no Mott transition at $x=0$, and the physics is dominated by an AFM transition at the VHS; for $U_{V}<U<U_{2}$, there is a Mott transition at half filling, which terminates (on the electron-doped side) before the plateau ends, and hence is controlled by dynamic critical exponent $z=2$; for $U_{2}<$ $U<U_{1}$, the Mott gap collapses in the enhanced regime near the edge of the plateau; and for $U>U_{1}$ the Mott gap terminates well off of the plateau, in a region of $z=1$ physics. For the present $\tau=-0.552$, the approximate values are $U_{V} / t=2.4, U_{2} / t=2.6$, and $U_{1} / t=3.6(x<0)$ or $4.1(x>0)$. Note that the $z=2$ regime is quite narrow, and can probably be subsumed into the VHS regime. These values depend on $t^{\prime}$, and the VHS moves to half filling as $t^{\prime} \rightarrow 0$, Fig. 33b. Even when $t^{\prime}=0$, Sen and Singh ${ }^{108}$ find a crossover from SDW-like to Heisenberglike behavior as correlations increase beyond $U_{0 \mathrm{~V}}=3.26 \mathrm{t}$ (diamond in Fig. 33b). It must be kept in mind that $U$ depends on doping, and the above estimates refer to $U$ near the plateau edge. The bare $U_{0}=U(x=0)$ can be estimated by assuming the doping is high enough to reach the Kanamori limit ${ }^{15}, U=U_{0} /\left(1+U_{0} / 8 t\right)$. This results in $U_{0 V} / t=3.4, U_{02} / t=3.8, U_{01} / t=6.5$ or 8.4 for $\tau=-0.552$. These last values are comparable to but somewhat smaller that those estimated by the first criterion.

Note that the cuprates are in the range $U_{2}<U<U_{1}$, where the plateau edges form natural phase boundaries for the Stoner criterion, thereby providing a natural explanation for the approximate electron-hole symmetry of the QCPs.

From the above discussion, it might be concluded that the cuprates are in the Slater regime, where mean field results are qualitatively accurate. While this is probably true for the electron-doped cuprates, it must be kept in mind that the criterion for the breakdown of the Slater regime is the flatness of the susceptibility in $\vec{q}$ - the inability of Slater theory to deal with many competing phases. In this light it is interesting to speculate whether the flatness of the $q$-plateaus might be a signal of enhanced Mott physics in the hole-doped cuprates.

\section{B. Magnon Bose Condensation and Non-Fermi Liquid Physics}

Figure 34 shows the sharp peak which arises in $\operatorname{Im} \Sigma$ at low $\mathrm{T}$. The growth is exponential, approximately matching that of the coherence length, Eq. 20. (Note that it requires a fine mesh in the integral of Eq. 28 to capture this growth.) This peak arises exactly at the incipient magnetic zone boundary, and turns into true Bragg scattering at the transition to long range order: the increase in peak height is almost exactly compensated by a decrease in the width of the peak. A simple physical explanation is that the SDW transition can be interpreted as a Bose condensation of the zone boundary magnons. Then the Mermin-Wagner theorem reduces to the fact that in a two-dimensional system, Bose particles can only condense at $T=0$. A similar explanation for the transition has been presented earlier ${ }^{3}$.
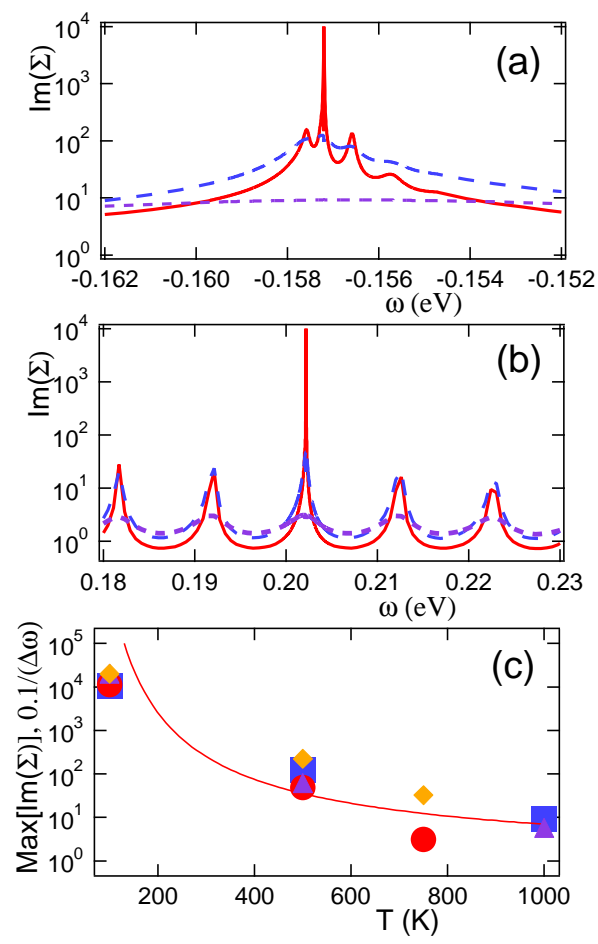

FIG. 34. (a,b): Blowups of $\operatorname{Im}(\Sigma)$ for $x=0$ at $(\pi, 0)$ (a) and $(\pi / 2, \pi / 2)$ (b) at $T=100 \mathrm{~K}$ (solid lines), 500K (long dashed lines), and $1000 \mathrm{~K}$ (a) or $750 \mathrm{~K}$ (b) (short dashed lines). (c) Maximum of $\operatorname{Im}(\Sigma)$ vs $T$ for $(\pi, 0)$ (squares) and $(\pi / 2, \pi / 2)$ (circles); $0.1 /$ (full width at half maximum) for $(\pi, 0)$ (triangles) and $(\pi / 2, \pi / 2)$ (diamonds); solid line $=$ corresponding $\xi(T)$, Eq. 20 .

In turn, the soft zone-boundary phonons explain one origin of non-Fermi liquid physics in the model: Bragg scattering from a fluctuating diffraction grating. How does one define Luttinger's theorem when the unit cell is strongly fluctuating?

\section{Comparison with Other Calculations}

As noted above, the present calculations predict that at the magnetic QCP the (possibly $T=0$ ) Neel phase will terminate, the Mott gap will collapse, and the Fermi 
pockets will merge into a large Fermi surface. This result distinguishes the present calculations from many others in the literature. Here a number of Slater-like theories are discussed.

The present results are generally consistent with the $t-J$ model in the low doping regime. However, since the $t-J$ model cannot readily deal with both Hubbard bands simultaneously, it is not appropriate in the present analysis of electron doped cuprates, where (a) ARPES can detect both bands (at least up to the Fermi level), and (b) the Mott gap is found to collapse with doping, leading to an overlapping of both bands at the Fermi level. In the $t-J$ model double occupancy is forbidden in the LHB, while in the UHB empty sites are forbidden. Moreover, the Hubbard model only allows $J$ values for $J \leq 1$ (Figs. 25, 27), and near this upper limit significant modifications are needed. In the SCBA approach to the $t-J$ model, the parameter $A_{1}$ has a broad peak ${ }^{62}$ when $J \sim 0.8$, not found in the Hubbard model SCBA calculations, suggesting that $t-J$ and Hubbard can be equivalent only for $J<<0.8$ or for $U>>5$ - that is, near half filling only. It is interesting to note that a recent $t-t^{\prime}-t^{\prime \prime}-J$ model calculation seems consistent with the first doped carriers forming weakly interacting quasiparticles in pockets of the respective upper or lower Hubbard bands, for either electron or hole doping ${ }^{109}$.

The NAFL and spin fermion models are also based on Slater-type physics, and should in principle make similar predictions to the present SCR model. However, they tend to take their parameters from experiment, which can lead to complications in the presence of stripe phases. For example, for hole doping, long range Neel order and diverging susceptibilities terminate at a very low doping, $x \sim 0.02$. While the SCR model predicts $x_{Q C P} \sim 0.25$, some empirical models take $x_{Q C P} \sim 0.02$. In this case, the QCP is divorced from Mott gap, since the Mott gap will clearly persist above $x=0.02$. Even worse, Matsuda, et al. ${ }^{86}$ have shown that for doping between $x=0.02$ and half filling the system is phase separated, so uniform AFM order exists only at $x \leq 0$.

Three examples of spin fermion calculations will be given, to highlight the differences and similarities. (1) Abanov et al. ${ }^{110}$ postulate a small- $x$ magnetic QCP. They find that the magnetic resonance mode frequency goes to zero at this QCP, but also the superconducting gap vanishes at the same doping, which would have important consequences for the mechanism of superconductivity. This is in sharp contrast to the present model, where the magnetic QCP is at much higher doping. In this model, the low- $x$ QCP is superconducting, presumably associated with stripe effects. (2) Chubukov and Morr ${ }^{55,111}$ studied the crossover from small to large Fermi surfaces here driven at fixed doping by reducing $U$. They state ${ }^{111}$ that, "as the system moves away from half filling, the spectral weight transfers from the upper band into the lower band and, near optimal doping, there exists just one coherent band of quasiparticles." This suggests that the crossover is due to a spectral weight shift, and not to the gap closing - in contrast to the present results and to experiment on NCCO. However, it should be noted that (a) their paper actually concentrates on changes at the Fermi level, and did not explore how the UHB might have shifted with $U$; and (b) it is possible that the coherent part of the UHB collapses, while some weight remains in the incoherent part. (3) On the other hand, Schmalian, et al. ${ }^{56}$ go beyond the SCBA, summing both non-crossing and crossing diagrams via a generalization of a technique of Sadovskii ${ }^{112}$; their results for hole doping are quite similar to the present results, with a magnetic QCP above optimal doping - but with $\xi$ adjusted at each doping to fit the experiment.

A number of groups have studied the Hubbard model using FLEX calculations, and have had considerable success in describing anomalous transport properties ${ }^{113}$. Here a pseudogap is found even though the FLEX model cannot describe the splitting into UHB and LHB, and the pseudogap is derived from superconducting fluctuations. However, these models are consistent with the present results, in that (1) the pseudogap they describe is clearly the lower, leading edge pseudogap which is not described by the present model, and (2) their calculation of the normal state properties require a value of $U / t \sim 1.5-2.5$ much smaller than the values found at half filling, and comparable to (or even smaller than) the doped values found here. [Spin fermion calculations also extract a small value of $U$ - there called $g$ - from experiments in near-optimally hole doped cuprates ${ }^{114}$.]

The present calculations are in general consistent with the results of Ref 3. These authors employ a (TwoParticle Self Consistent) conserving approximation, and attempt to calculate $U(x)$ directly $^{115}$. However, they incorporate the strong thermal (Mermin-Wagner) fluctuations directly into their definition of $U$, so the resulting doping dependence should not be compared to the form assumed here.

A leading edge pseudogap can also arise in the Hubbard model in the absence of superconductivity ${ }^{116,67,14}$, but only for large $U>8 t^{14}$.

Some recent calculations have confirmed that $U$ must decrease with electron doping to reproduce the ARPES data: in Kusonose and Rice ${ }^{13}$ the demonstration is indirect - the gap collapse does not occur in a SCBA calculation if $U$ is kept large. Sénéchal and Tremblay ${ }^{14}$ give a more direct demonstration; their model can also explain the hole-doped pseudogap near $(\pi, 0)$ in the absence of stripe physics if $U$ does not decrease with hole doping.

Finally, a proper study of the model incorporating QCP fluctuations is a strong desideratum, but the problem of combining QCP and Mermin-Wagner fluctuations has rarely ${ }^{117}$ been tackled in the literature. 


\section{VHS}

Whether or not the VHS is responsible for the observed electron-hole asymmetry, the present calculations reveal some novel features of Van Hove physics.

\section{Temperature Dependent VHS}

As noted by Onufrieva and Pfeuty ${ }^{43}$, the VHSs associated with the susceptibilities (and hence with charge or spin nesting) are different from those associated with the density of states (and superconductivity). Thus, whereas superconductivity will occur at the same optimal doping for all temperatures, the doping of maximal nesting instability is a strong function of temperature.

This contrasting behavior of nesting vs pairing susceptibilities is related to a characteristic difference in the nature of the two instabilities. A superconducting instability has an intrinsic electron-hole symmetry, which means that the gap is tied to the Fermi level, and a full (s- or d- wave) gap can be opened at any doping level. On the other hand, a nesting gap is dispersive, and only part of it lies at the Fermi level (except in special cases). Furthermore, a (superlattice) Luttinger's theorem must be obeyed, requiring the presence of residual Fermi surface pockets. Stated differently, a full nesting gap can only open at integer filling, so as the interaction strength increases, any nesting instability must migrate to integral doping (e.g., half filling in the original band structure). This same VHS migration is mirrored in the Tdependence of the magnetic (or charge) susceptibility.

\section{VHS Transitions}

We have seen that the doping-dependent $U_{\text {eff }}$ gives rise to a Mott gap collapse near the edges of the susceptibility plateau in Fig. 1. If $U_{\text {eff }}$ is smaller (dot-dashed line: $U_{\text {eff }}$ reduced by $2 / 3$ ), more complicated behavior should arise. Due to the peak in $\chi$ near the H-point, there could be a reentrant transition, with one magnetic order near half filling, and a second near the VHS. For an even smaller $U_{\text {eff }}$ (or replacing $\left.U_{\text {eff }} \rightarrow J\right)^{43}$, the transition near $x=0$ can be eliminated, leaving a spin density wave transition near the VHS. In principle there could even be a phase separation between two AFM phases: an insulating phase near half filling and a metallic phase near the VHS.

\section{CONCLUSIONS}

The key conclusion to this work can be stated as follows: In doped cuprates there is a magnetic QCP where three factors coincide: the crossover from small to large Fermi surface, Mott gap collapse, and Neel transition termination. In the SCR calculation there is no finite temperature Neel transition, at least in the isotropic $2 \mathrm{~d}$ limit, but the zero-temperature Neel transition persists with doping up to a QCP controlled by a modified Stoner criterion. While the Mott gap opening is more of a crossover than a sharp transition, nevertheless, the upper and lower Hubbard bands merge at nearly the same point, and the Fermi surface pockets recombine to a single large Fermi surface, consistent with band structure calculations. Comparison with experiment suggests that this correctly describes the situation in electron doped NCCO, both in ARPES (Section IV) and in magnetization studies, Ref. 83 and Section VII.

The hole doped case also appears to fit this model, but with complications associated with the $q$-plateau. Thus, (a) the pseudogap collapses in a QCP, as expected; (b) evidence for the Fermi surface crossover has recently been reported $^{79,78}$; (c) the correlation length appears to diverge as $T \rightarrow 0$, but much more weakly than for electron doping, due to a sum rule saturation ${ }^{80}$.

In more detail, the main results of this paper can be summarized:

- Fluctuation effects were added to the mean field Hubbard model via a mode coupling calculation, which allowed satisfying of the Mermin-Wagner theorem $\left(T_{N}=\right.$ $0)$. It was found that the mean-field gap $\Delta_{m f}$ and Néel temperature $T_{N}^{m f}$ evolved into a pseudogap $\Delta_{p s} \sim \Delta_{m f}$ and an onset temperature $T^{*} \sim T_{N}^{m f}$ (as is familiar from the related CDW results).

- The resulting dispersions and Fermi surfaces are in excellent agreement with photoemission experiments on electron-doped cuprates $^{8}$, while the pseudogap seems consistent with ARPES and tunneling results in hole doped cuprates.

- Magnetic properties - saturation magnetization and coherence length - are also well fit by the same model. The good agreement between ARPES and direct magnetic measurements leaves little doubt that the (large) pseudogap is predominantly magnetic in origin.

- The zero-temperature Néel transition is controlled by a Stoner-like criterion, hence is sensitive to the bare susceptibility and in turn to the Fermi surface geometry (hot spots). This lead to an approximately electron-hole symmetric QCP near optimal doping (termination of hot spot regime), at which both zero temperature Néel transition and pseudogap transition simultaneously terminate.

- The model leads to a NAFL-type susceptibility, and the calculation of the NAFL parameters has been reduced to a calculation of the coupling parameters $U$ and $u$, the former having a significant doping (and possibly temperature) dependence. At present, $U(x)$ is estimated from experiment, and the mode coupling $u$ via consistency with the $t-J$ model. (A small portion of the renormalization of $U$ arises from quantum corrections to the Stoner criterion.)

- The present theory differs from conventional NAFL theory by the inclusion of two cutoff parameters, $q_{c}$ and 
$\omega_{c}^{-}$, which shrink to zero at either the H- or C-points. For example, $q_{c}$ is large near the H-point, but shrinks to zero at the C-point, causing the $A$ parameter to have a strong temperature dependence in the electron-doping regime.

- Finally, a striking temperature/frequency dependence of the VHS susceptibility peak ${ }^{43}$, causing it to shift to half filling at high $T$, is interpreted in terms of Luttinger's theorem: if the coupling is strong enough to open a full gap, the gap must fall at half filling.

Note: After the present work was completed, I received a preprint from A.-M.S. Tremblay reporting similar calculations for electron-doped cuprates ${ }^{118}$.

Acknowledgments: This work was supported by the Spanish Ministerio de Educación through grant SAB2000-0034, and by the U.S.D.O.E. Contract W-31109-ENG-38, and benefited from the allocation of supercomputer time at the NERSC and the Northeastern University Advanced Scientific Computation Center (NU-ASCC). Part of this work was done while I was on sabbatical at the Instituto de Ciencia de Materiales de Madrid, CSIC, Cantoblanco, E-28049 Madrid, Spain. I thank my hosts, Maria Vozmediano and Paco Guinea, for a very stimulating visit, for numerous discussions, and for correcting an error in the original calculation.

I thank Walter Harrison for stimulating conversations on calculating the interlayer coupling, and Martin Greven and Antonio Castro-Neto for useful comments on the magnetic properties, and A.-M.S. Tremblay for a preprint of his work.

\section{APPENDIX A: THREE BAND MODEL}

A major simplification of the present calculation is to treat the cuprates in a one-band model. This is consistent with the Zhang-Rice picture ${ }^{119}$, although the approximation is less drastic for electron doping, since the upper Hubbard band is already predominantly copperlike. Nevertheless, the model also describes the doping dependence of the 'lower Hubbard band', which is really a charge transfer, predominantly oxygen-like band. Here an explanation for why this simplification works is suggested.

Even without carrying out self-consistent calculations, the nature of the Mott transition can be understood by introducing a doping dependent gap. The energy bands can be calculated from the hamiltonian matrix

$$
\begin{aligned}
H=\sum_{j} \Delta d_{j}^{\dagger} d_{j}+ & \sum_{<i, j>} t_{C u O}\left[d_{j}^{\dagger} p_{i}+(c . c .)\right] \\
+ & \sum_{<j, j^{\prime}>} t_{O O}\left[p_{j}^{\dagger} p_{j^{\prime}}+(c . c .)\right] \\
& \left.+U n_{j \uparrow} n_{j \downarrow}+U_{p} n_{i \uparrow} n_{i \downarrow}\right),
\end{aligned}
$$

where $\Delta$ is the difference in on-site energy between copper and oxygen, $t_{\mathrm{CuO}}$ is the copper-oxygen hopping parameter, $t_{O O}$ the oxygen-oxygen hopping parameter and
$U\left(U_{p}\right)$ the Hubbard interaction parameter on $\mathrm{Cu}(\mathrm{O})$. For good agreement with the doping dependence of the one band model, it is necessary to properly incorporate the Hartree correction to the self energy, $\Delta=\Delta_{0}+\Sigma_{H}$, $\Sigma_{H}=U n_{\downarrow}$ (for up spins), and $n_{\downarrow}=n / 2-m_{Q}$, with $n$ the average electron energy. The resulting dispersions are shown in Fig. 16 for the antibonding bands, and Fig. 35 for the full dispersion. In these figures, the following parameters are assumed: $t_{C u O}=0.8 \mathrm{eV}, t_{O O}=-0.4 \mathrm{eV}$, $\Delta_{0}=0, U=6 \mathrm{eV}$, and $U_{p}=3.75 \mathrm{eV}$.

The band dispersion is extremely similar to that found in the one band model, Fig. 15, even though the lower band crosses over from the Zhang-Rice (hybridized copper-oxygen band) at half filling to a more copper like lower Hubbard band with increasing electron doping. In addition, the effective magnetizations are proportional, Fig. 36, although the one-band model overestimates the magnetization by $1 / 3$. This can be understood: in the three-band model, the shape of the Hubbard bands is fixed by the combined effects of the magnetic instability and hybridization with the oxygen band. In the one band model, only the former effect is present, necessitating a larger value of $m$ to produce the same net splitting.

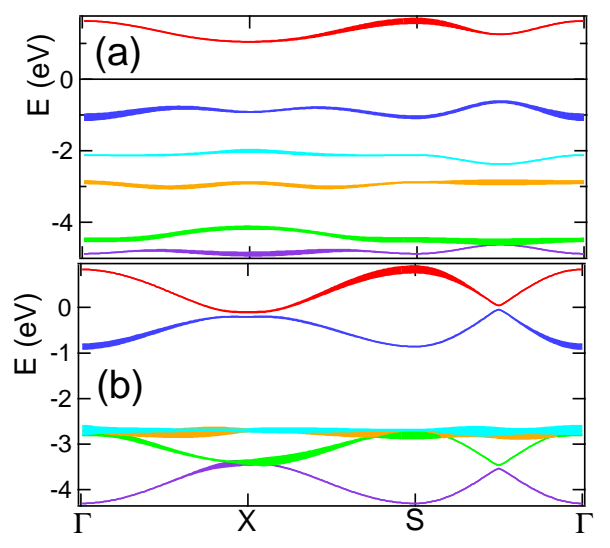

FIG. 35. Dispersion of all six bands in three-band model, assuming $m_{Q}=0.3(\mathrm{a})$ and $0.01(\mathrm{~b})$.

This remarkable agreement between one and threeband models goes well beyond the Zhang-Rice model. That model is restricted to the LHB in a small range of doping near half filling; the present results compare both LHB and UHB over the full range of electron doping. The result is nontrivial - in the three band model, the bonding and non-bonding bands are also split into upper and lower Hubbard bands. This degree of agreement comes about because the parameter $\Delta$ includes a large contribution from the magnetic Hartree term. In turn, this suggests that in the absence of magnetic effects the $\mathrm{Cu}$ and $\mathrm{O}$ energies are nearly degenerate - as found in early LDA band structure calculations (see discussion in Ref. 120). 


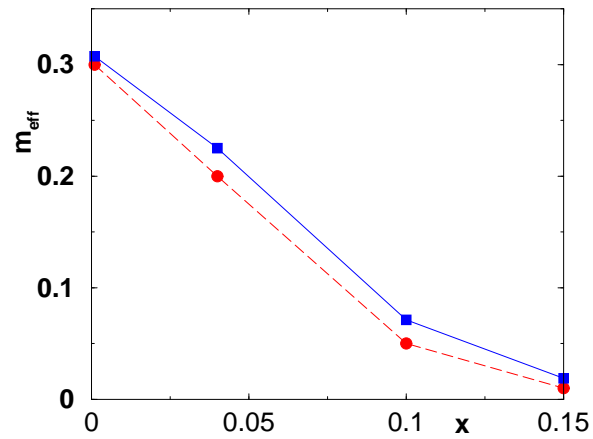

FIG. 36. Effective magnetization $m_{e f f}=m U / 6 t$ for the three-band (circles) and one-band (squares) models. The one-band result has been multiplied by $3 / 4$ to better agree with the three-band results.

\section{APPENDIX B: CHARGE SUSCEPTIBILITY AND $U_{E F F}$}

The proper choice of vertex corrections is an unresolved issue in the analysis of the Hubbard model. It is known to be of critical importance for generating a pseudogap $^{121}$. Here, by comparing simple mean-field and SCR models to experiment, it is shown that the net effect of vertex corrections is to make the coupling $U$ effectively doping (and possibly temperature) dependent. Kanamori ${ }^{15}$ showed that the effective Hubbard $U$ should decrease with doping, as an electron can hop around, and hence avoid, a second electron. In the limit of a nearly empty (or full) band, this should lead to a correction of the form $U_{\text {eff }} \sim U /(1+U / W)$, where $W=8 t$ is the bandwidth. It was found ${ }^{122,53}$ that Monte Carlo calculations of the susceptibility of a doped Mott insulator were approximately equal to the RPA susceptibility with suitable $U_{\text {eff }}$, and Chen, et al. ${ }^{122}$ suggested the explicit form $U_{\text {eff }}=U /(1+<P>U)$, with $\mathrm{P}$ given by a vertex correction to the susceptibility and $\langle\cdots\rangle$ an average over $\vec{q}$, at zero frequency. Figure $37 \mathrm{~b}$ presents a calculation for $U_{\text {eff }}$ based on Chen, et al. However, whereas Chen, et al. performed the average in the paramagnetic phase, using bare Green's functions, here the dressed Green's functions appropriate to the Néel phase are used, to approximately incorporate the effect of this gap. This makes little difference, since $P$ is dominated by the intraband terms, and remains finite at half filling. Explicitly,

$$
\begin{gathered}
P=-\frac{1}{N} \sum_{i, j, k} \hat{U}_{i, j}(k, k+q) \tilde{F}_{i, j}(k, k+q), \\
\tilde{F}_{i, j}\left(k, k^{\prime}\right)=\frac{1-f_{k}^{i}-f_{k^{\prime}}^{j}}{E_{i}(\vec{k})+E_{j}\left(\vec{k}^{\prime}\right)-\omega-i \delta}, \\
E_{ \pm}(\vec{k})=\frac{1}{2}\left(\epsilon_{k}+\epsilon_{k+q} \pm E_{0}\right),
\end{gathered}
$$

$$
\begin{gathered}
E_{0}=\sqrt{\left(\epsilon_{k}-\epsilon_{k+q}\right)^{2}+4 \Delta^{2}}, \\
\hat{U}_{i, j}\left(k, k^{\prime}\right)=\frac{1}{4}\left(1+i A_{k}\right)\left(1+j A_{k^{\prime}}\right)+i j B_{k} B_{k^{\prime}},
\end{gathered}
$$

with $i, j$ summed over,,$+- \Delta$ the AFM gap, and $A_{k}=$ $\left(\epsilon_{k}-\epsilon_{k+Q}\right) / E_{0 k}, B_{k}=\Delta / E_{0 k}$. In agreement with Chen, et al., the calculation finds $U$ to be renormalized by a factor of 2 at finite doping, but does not recover a large $U$ near half filling, although different results are found depending on whether $x=0$ from the start (triangle) or whether $x \rightarrow 0$ from the hole or electron doping sides.

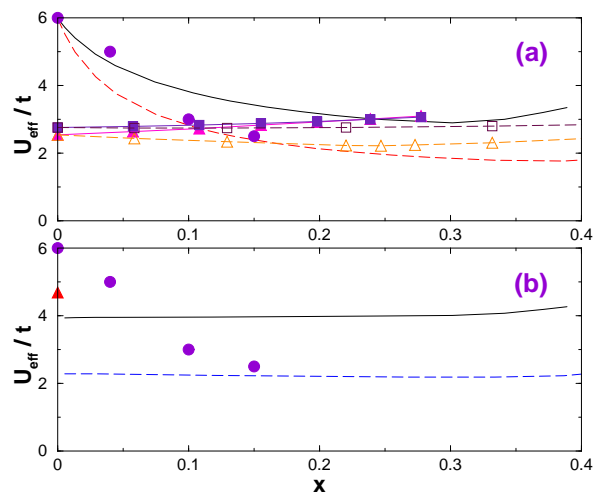

FIG. 37. Calculated $U_{\text {eff }}$ assuming (a) simple screening or (b) full vertex correction of Chen, et al. [122]. In both cases, a bare $U=6.75 t$ was assumed. Solid lines = electron doping; long dashed lines $=$ hole doping; triangles (squares) in $\mathrm{a}=$ paramagnetic screening of $U$, at $T=1 \mathrm{~K}(2000 \mathrm{~K})$; triangle in $\mathrm{b}=$ undoped; circles $=$ data of Ref. [ 8].

For modelling purposes, it is useful to have a $U_{\text {eff }}$ which evolves smoothly from a large value at half filling to a reduced, Kanemori value at finite doping. A simple toy model consists of taking the RPA screening of a charge response. There should be a close connection between the Kanemori mechanism and screening. Screening involves creation of a correlation hole about a given charge, while Kanemori's $U_{\text {eff }}$ involves the ability of a second charge to move around the first, while avoiding double occupancy. Near half filling, the second charge must move in the correlation hole. Approximating ${ }^{9}$ the vertex correction by the RPA screening of the charge susceptibility,

$$
U_{e f f}=\frac{U}{1+<\chi>U},
$$

it is possible to reproduce ${ }^{9}$ the experimentally observed ${ }^{8}$ doping dependence, while matching the calculation of Chen, et al. away from half filling, Fig. 37a.

In this calculation, issues of self-consistency are also important. To minimize screening at half filling, it is necessary to reproduce the gap in the susceptibility. Hence, the susceptibility in Eq. B6 is approximated by the charge susceptibility in the AFM state, $\bar{\chi}_{0}^{00}$ from Eq. 2.24 of 
Ref. 1, evaluated with the bare $U=6.75 t$. (In principle, at finite doping there is a coupling to the longitudinal magnetic susceptibility ${ }^{63}$, but this is neglected for simplicity.) The importance of using the AFM susceptibility is illustrated in Fig. 37a: the solid and dashed lines show $U_{\text {eff }}$ calculated using the charge susceptibility in the Néel state, while the corresponding lines with triangles use the paramagnetic susceptibility at low $T$. The latter calculation finds a nearly doping independent, but small $U_{\text {eff }}$; the former reproduces a large, weakly screened $U$ near half filling. Such a difference is expected in terms of screening: when there is no gap at half filling, the enhanced susceptibility should be better able to screen $U$, resulting in a smaller $U_{\text {eff }}$. This suggests that $U_{\text {eff }}$ should have an important temperature dependence as the gap decreases - which in turn will cause the gap to close at a lower temperature. Figure 37a also shows that there is a weak temperature dependence of the screening. The calculations suggest that the large values of $U$ found in the cuprates are characteristic mainly of the half filled regime and relatively low temperatures. A similar but larger screening effect was recently reported by Esirgen, et al. ${ }^{123}$.

This procedure is still not fully self consistent. If there is a large difference between the bare $U$ and the screened $U_{\text {eff }}$, the gap in $\chi$ should depend on the actual $U_{\text {eff }}$. However, since $U_{\text {eff }} \simeq U$ at half filling, any simple improvement will not significantly change the overall doping dependence. This is the same kind of lack of selfconsistency found for the SCR approach, and will be here neglected.

\section{APPENDIX C: IMPROVED SOLUTION OF SCR EQUATION}

Approximating $\operatorname{coth}(x)=\max (1 / x, 1)$, and introducing the notation $\bar{A} q_{c}^{2}=A q_{c}^{2}+\delta, \bar{a}_{q}=\bar{A} q_{c}^{2} / \alpha_{\omega}$, and $t=2 T C$, the solution to Eq. 6 becomes

$$
\delta-\delta_{0}=\frac{3 u a^{2}}{\pi^{2} A C}\left[F_{1}+F_{2}\right],
$$

with

$$
\begin{gathered}
F_{1}=\int_{\delta}^{\delta+A q_{c}^{2}} d y \int_{t}^{\alpha_{\omega}} d x \frac{x}{x^{2}+y^{2}}= \\
\frac{\bar{A} q_{c}^{2}}{2} \ln \left[\frac{1+\bar{a}_{q}^{2}}{\bar{a}_{q}^{2}+\left(t / \alpha_{\omega}\right)^{2}}\right]+ \\
\alpha_{\omega} \tan ^{-1}\left(\bar{a}_{q}\right)-\frac{\delta}{2} \ln \left[\frac{\delta^{2}+\alpha_{\omega}^{2}}{\delta^{2}+t^{2}}\right]-\alpha_{\omega} \tan ^{-1}\left(\frac{\delta}{\alpha_{\omega}}\right) \\
F_{2}=t \int_{\delta}^{\delta+A q_{c}^{2}} d y \int_{0}^{t} \frac{d x}{x^{2}+y^{2}}= \\
=t \int_{\delta}^{\delta+A q_{c}^{2}} \frac{d y}{y} \tan ^{-1}\left(\frac{t}{y}\right)=
\end{gathered}
$$

$$
=t\left[I_{1}\left(\frac{t}{\bar{A} q_{c}^{2}}\right)-I_{1}\left(\frac{t}{\delta}\right)\right]
$$

with

$$
\begin{gathered}
I_{1}(x)=I_{0}\left(\tan ^{-1}(x)\right)-\tan ^{-1}(x) \ln (x), \\
I_{0}(x)=\int_{0}^{x} \ln (\tan \theta) d \theta=L(x)+L\left(\frac{\pi}{2}-x\right)-L\left(\frac{\pi}{2}\right),
\end{gathered}
$$

and $L(x)=-\int_{0}^{x} \ln (\cos t) d t$ is the Lobachevskiy function $^{124}$.

For most purposes, it can be assumed that $\delta<<t<<$ $A q_{c}^{2}, \alpha_{\omega}$, in which case $I_{0}\left(\tan ^{-1}(x)\right)=\theta(\ln (\theta)-1)$, with $\theta=\min \{x, 1 / x\}$, and then $F_{2}$, Eq. C3, simplifies.

$$
\begin{aligned}
F_{2}=\ln \left(\frac{t}{\delta}\right)\left[\delta+\tan ^{-1}\left(\frac{t}{\delta}\right)\right] & +\delta-\frac{t^{2}}{A q_{c}^{2}} \\
& \simeq \frac{\pi}{2} t \ln \left(\frac{t}{\delta}\right) .
\end{aligned}
$$

Defining $Z=1+\left(3 u a^{2} / \pi^{2} A C\right) \ln \left(\alpha_{\omega} / t\right)$, then

$$
Z \delta-\bar{\delta}_{0}=\frac{3 u a^{2} T}{\pi A} \ln \left(\frac{2 C T}{\delta}\right),
$$

which agrees with Eq. 13 when $Z \rightarrow 1$.

\section{APPENDIX D: PARAMETER EVALUATIONS}

At $T=0$, the imaginary part of the susceptibility $\chi(\vec{Q}, \omega)$ can be calculated analytically:

$$
\begin{array}{r}
\operatorname{Im}(\chi(\vec{Q}, \omega))=\sum_{\vec{k}}\left(f\left(\epsilon_{\vec{k}}\right)-f\left(\epsilon_{\vec{k}+\vec{Q}}\right)\right) \delta\left(\epsilon_{\vec{k}+\vec{Q}}-\epsilon_{\vec{k}}-\omega\right) \\
=\frac{F\left(\theta_{1}, \tilde{k}\right)-F\left(\theta_{2}, \tilde{k}\right)}{4 t},
\end{array}
$$

where $F(\theta, x)$ is an elliptic integral, $\tilde{k}=\sqrt{1-(\omega / 8 t)^{2}}$, and $\sin \left(\theta_{i}\right)=\sin \left(\phi_{i}\right) / \tilde{k}$, with

$$
\begin{aligned}
& \cos ^{2}\left(\phi_{1}\right)=\left\{\begin{array}{ll}
c_{-}^{2} & \text { if } \omega \leq \omega_{c}^{-} \\
\hat{\omega} / 2 & \text { if } \omega>\omega_{c}^{-}
\end{array},\right. \\
& \cos ^{2}\left(\phi_{2}\right)= \begin{cases}c_{+}^{2} & \text { if } \omega \leq \omega_{0} \\
1 & \text { if } \omega>\omega_{0}\end{cases}
\end{aligned}
$$

with $\hat{\mu}=\mu / 2 t, \hat{\omega}=\omega / 4 t, c_{ \pm}^{2}=a_{ \pm}+\sqrt{a_{ \pm}^{2}-\hat{\omega}^{2}}$, and $a_{ \pm}=1-(\hat{\mu} \pm \hat{\omega}) / \tau$. Similar results for $t^{\prime}=0$ are discussed in Ref. 40. The real part $R e \chi$ can be found from the Kramers-Kronig result,

$$
\operatorname{Re\chi }(\vec{Q}, \omega)=\frac{1}{\pi} \int_{0}^{\infty} \frac{\operatorname{Im} \chi\left(\vec{Q}, \omega^{\prime}\right) \omega^{\prime} d \omega^{\prime}}{\omega^{\prime 2}-\omega^{2}} .
$$




\section{1. $A$ at the C-point}

To understand the $q$-plateau, and in particular the Cpoint, where the plateau width shrinks to zero, it is convenient to introduce a simplified $\operatorname{model}^{125}$, for which the $q$-dependence of $\chi$ can be calculated analytically. While the dashed lines in Fig. 41 represented an $\omega$ shift, they can equally well describe the $q$-shift of the energy denominator, Eq. 3. The plateau edge corresponds to the point where the dashed line intersects the q-shifted FS (horizontal arrows). (Recall that $\vec{q}=\vec{Q}+\vec{q}^{\prime}$.) In the simplified model, the energy denominator is linearized, so $\Delta \epsilon \propto k_{\perp}$, independent of $k_{\|}$. Chosing $\vec{q}$ to point along the $(\pi, \pi)$ direction, the FS can be approximated by two circles of radius $k_{F}$, centered at $(\pi, \pi)$ and $(-\pi,-\pi)$ (for this choice of $\vec{q}$ the other two circles at $(\pi,-\pi)$ and $(-\pi, \pi)$ can be ignored). The Q-shifted FS is then a circle centered at $\Gamma=(0,0)$. The FS at $(\pi, \pi)$ and the Q-shifted FS are illustrated in Fig. 38c. To keep the picture symmetrical, both FSs are shifted (in opposite directions) by $q^{\prime} / 2$ when $q^{\prime} \neq 0$.

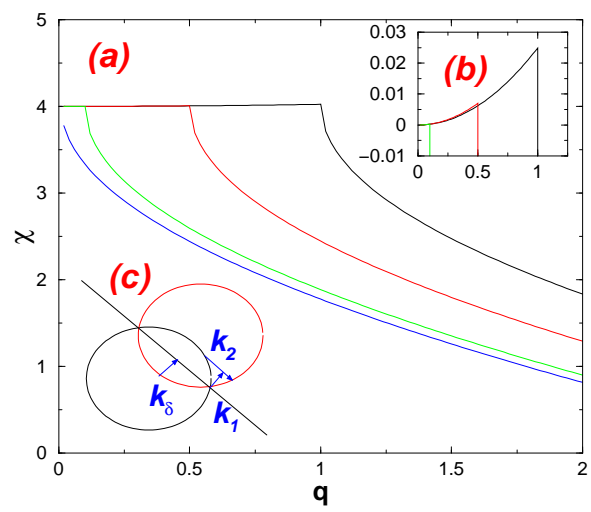

FIG. 38. (a) Calculated susceptibility $\chi(q)$ for several values of overlap $\delta$. (b) Blowup of plateau region, for $\chi_{q}-\chi_{q=0}$. (c) Model of Fermi surfaces, defining $\delta, k_{1}\left(k_{\perp}\right)$ and $k_{2}\left(k_{\|}\right)$.

Adding the contributions of the overlap of the q-shifted FS with both the FS at $(\pi, \pi)$ and the one at $(-\pi,-\pi)$, $\chi_{q} \propto I_{k_{\delta}+q^{\prime} / 2}+I_{k_{\delta}-q^{\prime} / 2}$, with

$$
I=\int_{0}^{k_{c}} \frac{d k_{\perp} d k_{\|}}{k_{\perp}},
$$

where the region of integration is over the part of the upper FS in Fig. 38c not overlapped by the lower (qshifted) $\mathrm{FS}$, and $k_{\perp}$ ranges from zero at the apex of the wedge to the middle of the upper FS, $k_{c}=k_{F}-k_{\delta}$, where $k_{\delta}$ is the overlap parameter defined in Fig. 38c. To lowest order, for $k_{\delta}<<k_{F}$,

$$
I=2 k_{F}+\sqrt{k_{F} k_{\delta}} \ln \left|\frac{1-\beta}{1+\beta}\right|
$$

with $\beta=\sqrt{k_{\delta} / k_{F}}$. The expression for $I_{k_{\delta}-q^{\prime} / 2}$ must be modified when $q^{\prime}>2 k_{\delta}$ and the two FSs no longer overlap ${ }^{125}: I_{k_{\delta}-q^{\prime} / 2}=2 k_{F}\left[1-\gamma \tan ^{-1} 1 / \gamma\right]$, with $\gamma=\sqrt{\left(q^{\prime}-2 k_{\delta}\right) / 2 k_{F}}$. The calculated susceptibilities, Fig. 38a, display the flat topped plateaus with weak positive curvature $(A<0$, Fig. 38b). At the plateau edge the susceptibility falls sharply, $\chi \sim 1-\pi \gamma / 2 \sim \sqrt{q^{\prime}}$. The C-point corresponds to $k_{\delta}=0$.

\section{2. $C$ and Plateaus in Frequency}
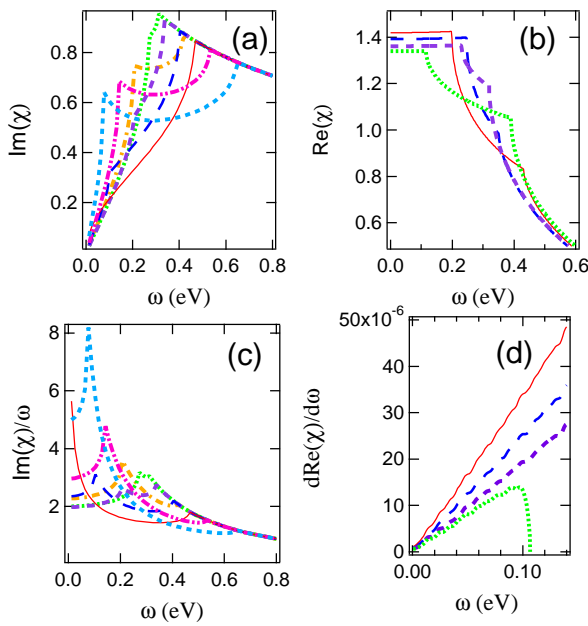

FIG. 39. (a) $\operatorname{Im} \chi(\vec{Q}, \omega)$, (b) $\operatorname{Re} \chi(\vec{Q}, \omega),($ c) $\operatorname{Im} \chi / \omega \equiv \hat{C}$, and (d) $d \operatorname{Re} \chi(\vec{Q}, \omega) / d \omega$, for (a,c): $\mu=0$ (solid line), -0.05 (long dashed line), -0.10 (dashed line), -0.15 (dotted line), -0.20 (dot-dashed line), -0.25 (dot-dot-dashed line), and $-0.30 \mathrm{eV}$ (short dashed line); (b,d): $x=0$ (solid line), 0.04 (long dashed line), 0.10 (dashed line), and 0.15 (dotted line).

Figure 39 illustrates $\operatorname{Im} \chi(\vec{Q}, \omega), \operatorname{Re} \chi(\vec{Q}, \omega)$, and $\operatorname{Im} \chi / \omega \equiv \hat{C}$. While plateaus in $\operatorname{Re}\left(\chi_{0}\right)$ have been noted above, Fig. 1c, here the main interest lies in $C=$ $U \operatorname{Im} \chi / \omega$. This linear-in-frequency contribution to $\operatorname{Im} \chi$, generated by hot spots, is an important parameter in SCR and NAFL theories, and has been well studied. The height of the plateau at zero frequency $C=U \hat{C}(\omega=0)$ can be represented as a frequency $\omega_{1}=1 / C$, which can be found explicitly ${ }^{52}$

$$
C=\frac{1}{2 \pi J s_{x 0}^{2}\left(1+\tau c_{x 0}\right)}=\frac{1}{\omega_{1}}
$$

(with $J=4 t^{2} / U, s_{x 0}^{2}=1-c_{x 0}^{2}$ ).

However, it is important to note that $C$ also approximates a plateau, particularly near the H-point, Fig. 40, with a well-defined cutoff. Moreover, the width of this plateau vanishes near both the $\mathrm{H}$ - and C-points, controlled by two characteristic frequencies, $\omega_{c}^{-}$, Eq. 24, and

$$
\omega_{0}=\frac{8 t}{\tau}[\sqrt{1-\hat{\mu} \tau}-1],
$$


respectively. The origin of these critical frequencies can be understood from Fig. 41. The thick (thin) solid lines represent the original (Q-shifted) Fermi surfaces, while the dashed lines represent

$$
\omega=\epsilon_{\vec{k}+\vec{Q}}-\epsilon_{\vec{k}}
$$

for various values of $\omega$. Equation D9 gives the points at which the denominator of $\chi_{0}(\vec{Q}, \omega)$, Eq. 3, vanishes. Thus at $T=0, \operatorname{Im}\left(\chi_{0}(\vec{Q}, \omega)\right)$ is proportional to the length of the dashed line lying between the original and Q-shifted FSs (i.e., where $\left.f\left(\epsilon_{\vec{k}}\right)-f\left(\epsilon_{\vec{k}+\vec{q}}\right)= \pm 1\right)$. Since the two FSs meet at an angle, forming a wedge, $\operatorname{Im}\left(\chi_{0}(\vec{Q}, \omega)\right) \sim \omega$.

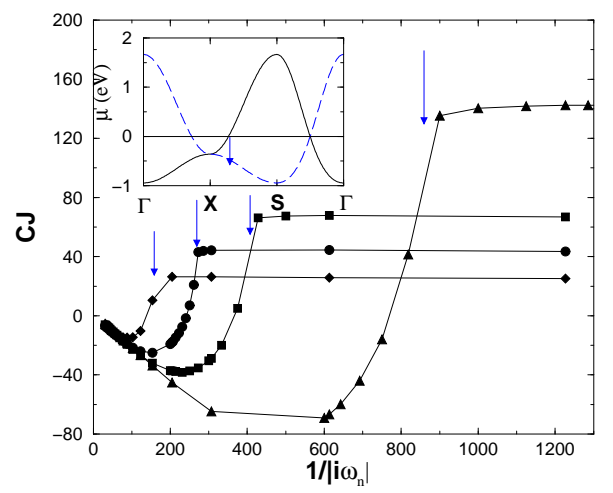

FIG. 40. $\hat{C}$ calculated for several values of $\mu$ : $\mu=-0.355$ (diamonds), -0.357 (circles), -0.358 (squares), $-0.359 \mathrm{eV}$ (triangles) $\left[\mu_{v}=-0.3599 \mathrm{eV}\right.$. Inset: Band dispersion $\epsilon_{\vec{k}}$ (solid line) $\epsilon_{\vec{k}+\vec{Q}}$ (dashed line), for $\mu=0$. Arrow $=\omega_{c}^{-}$.

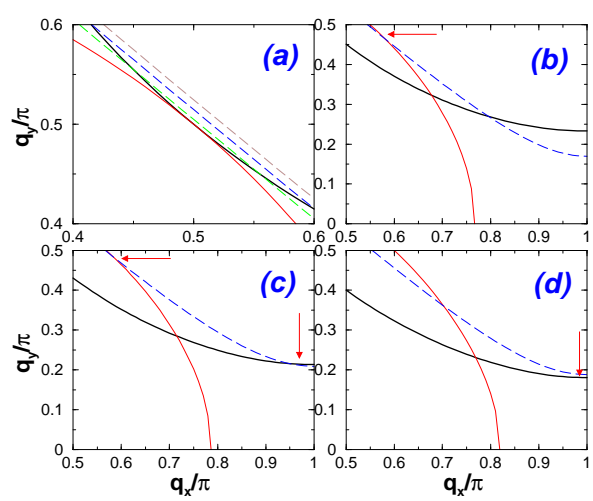

FIG. 41. Origins of critical cutoffs. Thick solid line = FS; thin solid line = Q-shifted FS; dashed lines = Eq. D9, for several values of $\omega$. Chemical potential $\mu=$ (a) 0 , (b) -0.1 , (c) $-0.14,(\mathrm{~d})-0.2 \mathrm{eV}$. horizontal arrows indicate $\omega_{0}$, vertical arrows $\omega_{c}^{-}$.

From Fig. 41, the critical frequencies (denoted by arrows) are points where the $\omega$ dependence of this length changes abruptly, leading to a sharp change in $\operatorname{Im} \chi$. Thus, near the H-point, the plateau width is $\omega_{c}^{-}$(in- set, Fig. 40), while near the C-point it is $\omega_{0}$. The vertical arrows in Fig. 41 indicate $\omega_{c}^{-}$, where the dashed line (Eq. D9) intersects the FS at the zone boundary, while the horizontal arrows $\operatorname{are}^{40} \omega_{0}$, where the dashed line ceases to intersect the Q-shifted FS. There is a crossover at $\mu_{c} \simeq-0.14 \mathrm{eV}$ : for $\mu>\mu_{c}, \omega_{0}=\omega_{c}^{-}$ while for $\mu<\mu_{c}, \omega_{0}=\omega_{c}^{-}$. Combining Eqs. 24,D8, $\omega_{0}=\omega_{c}^{-}$at $\mu_{c}=\left[1-z(2-\sqrt{z})^{2}\right] 2 t / \tau=-0.1384 \mathrm{eV}$, with $z=1-\tau$. For $\omega>\min \left\{\omega_{0}, \omega_{c}^{-}\right\}, \operatorname{Im}\left(\chi_{0}(\vec{Q}, \omega)\right) \sim \omega^{1 / 2}$, so $C \sim 1 / \omega^{1 / 2}$ - i.e., the susceptibility is no longer on the plateau.

Defining a width parameter $\alpha_{\omega}=\min \left\{\alpha_{\omega}^{-}, \alpha_{\omega}^{0}\right\}$, with $\alpha_{\omega}^{0}=\omega_{0} / \omega_{1}$, then

$$
\omega_{1} / \omega_{c}^{-}=\frac{2 \pi t(1-\tau)}{U}\left[\frac{1+\tau c_{x 0}}{-\tau}\right] \equiv \frac{1}{\alpha_{\omega}^{-}} .
$$

This latter is in good agreement with the numerical results (arrows in Fig. 41) and is similar to the result found by Onufrieva and Pfeuty ${ }^{43}$, using a hyperbolic band approximation valid near a VHS, $\omega_{1} / \omega_{c}^{-}=2 \pi t(1-\tau) / U$.

Because of the dynamic scaling $\omega \sim q^{z}$, this crossover is also reflected in the behavior on the plateau in $\vec{q}$, Fig. 7: for $\mu>-0.14 \mathrm{eV}$, the plateau has a negative curvature, which can almost be scaled between different dopings, while for $\mu<-0.14 \mathrm{eV}$, the plateau starts to fill in, ultimately developing a peak at $\vec{Q}$. (See also Fig. 3a in Ref. 12.) Note that the plateau width collapses in frequency at both the $H$ - and $C$-points, while the collapse in wave number $\left(q_{c} \rightarrow 0\right)$ is only present near the $C$-point.

3. $B$

The parameter $B$ is small, and generally neglected. However, it enters into the evaluation of $u$, so will be discussed briefly. The expression for $B$ may be written exactly as the $\omega \rightarrow 0$ limit of

$$
B=U R e \sum_{\vec{k}}\left[\frac{f\left(\epsilon_{\vec{k}}\right)-f\left(\epsilon_{\vec{k}+\vec{Q}}\right)}{\left(\epsilon_{\vec{k}+\vec{Q}}-\epsilon_{\vec{k}}\right)}\right] \frac{1}{\left(\left(\epsilon_{\vec{k}+\vec{Q}}-\epsilon_{\vec{k}}\right)^{2}-\omega^{2}\right.} .
$$

It can be shown that $B$ has a logarithmic correction due to the hot spots. The integral can be approximately evaluated by (a) using symmetry to reduce the integral to one over an octant of the Brillouin zone containing one hot spot, (b) splitting the domain of integration into (i) a circle of radius $k_{c}$ about the hot spot, and (ii) the remainder of the domain, and (c) numerically evaluating the integral over domain (ii) while providing an analytic approximation to that over (i). Then the $k$ integral over the hot spot circle can be written approximately as

$$
\begin{array}{r}
I=\int_{0}^{k_{c}} \frac{\left(1-3 \beta_{\theta} k\right) d k}{\alpha_{\theta}^{2} k^{2}-\omega^{2}} \\
\simeq \frac{1}{\alpha_{\theta}^{2}}\left[\frac{1}{k_{c}}-3 \beta_{\theta} \log \frac{\alpha_{\theta} k_{c}}{\omega}\right] .
\end{array}
$$


At $T=0$, the integral $I$ must then be integrated in $\theta$ over the wedge where the difference in Fermi functions does not vanish. The integral from outside the hot spot circle will eliminate the $k_{c}$-dependence, but should not affect the $\log (\omega)$ term.

It is difficult to directly evaluate the two-dimensional principal value integral for $B$. Instead, it is much simpler to evaluate $R e(\chi)$ via Kramers-Kronig transformation of $\operatorname{Im}(\chi)$ and find $B$ by numerical differentiation. When this is done, it is found that (a) $B$ is numerically very small due to the plateau in $R e(\chi)$, Fig. 39d, and (b) the logarithmic correction is too small to determine accurately.

\section{4. $u$}

The quartic effective action is

$$
\begin{array}{r}
S=\frac{1}{2} \sum_{\vec{q}, i \omega_{n}} \Pi_{2}\left(\vec{q}, i \omega_{n}\right) \phi\left(\vec{q}, i \omega_{n}\right) \phi\left(-\vec{q},-i \omega_{n}\right) \\
+\frac{1}{4\left(\beta N_{0}\right)^{2}} \sum^{\prime} \Pi_{4}\left(\vec{q}_{i}, i \omega_{i}\right) \phi\left(\vec{q}_{1}, i \omega_{1}\right) \phi\left(\vec{q}_{2}, i \omega_{2}\right) \times \\
\times \phi\left(\vec{q}_{3}, i \omega_{3}\right) \phi\left(\vec{q}_{4}, i \omega_{4}\right),
\end{array}
$$

where the prime in the second sum means summing over all $\vec{q}_{i}, \omega_{i}$, such that $\sum_{i=1}^{4} \vec{q}_{i}=0, \sum_{i=1}^{4} \omega_{i}=0$,

$$
\Pi_{2}\left(\vec{q}, i \omega_{n}\right)=\frac{U}{2}\left[1-U \chi_{0}\left(\vec{q}, i \omega_{n}\right)\right]
$$

$\Pi_{4}\left(\vec{q}_{i}, i \omega_{n}\right)=\frac{U^{4}}{8} \sum_{\vec{k}, i \epsilon_{n}} G_{0}\left(\vec{k}, i \epsilon_{n}\right) G_{0}\left(\vec{k}+\vec{q}_{1}, i \epsilon_{n}+i \omega_{1}\right) \times$
$\times G_{0}\left(\vec{k}+\vec{q}_{1}+\vec{q}_{2}, i \epsilon_{n}+i \omega_{1}+i \omega_{2}\right) G_{0}\left(\vec{k}-\vec{q}_{4}, i \epsilon_{n}-i \omega_{4}\right)$,

with $u=\Pi_{4} / N_{0} \beta U^{2}$.

Since there is some controversy ${ }^{36,33}$ concerning $u$, it shall be evaluated in detail. Millis ${ }^{36}$ showed that for free electrons (parabolic bands) this expression is in general well defined, but diverges when $\vec{Q}$ is a 'spanning' vector of the Fermi surface - in the present case, this would correspond to the $\mathrm{H}$ - and C-points. Abanov, et al. ${ }^{33}$ found a more severe divergence: $u$ diverges for all $\mu$ in the hot spot regime. The problem lies in the limit of external frequencies $\rightarrow 0$, momenta $\rightarrow 0$ or $\vec{Q}$. Taking this limit on the momenta, the expression for $u$ can be written as

$$
\begin{gathered}
u=\frac{U^{2}}{N_{0} \beta} \sum_{\vec{k}, i \omega_{n}} \frac{1}{\left(\epsilon_{\vec{k}}-i \omega_{n}\right)\left(\epsilon_{\vec{k}}-i \omega_{n}+i \omega_{4}\right)} \times \\
\times \frac{1}{\left(\epsilon_{\vec{k}+\vec{Q}}-i \omega_{n}-i \omega_{1}\right)\left(\epsilon_{\vec{k}+\vec{Q}}-i \omega_{n}-i \omega_{1}-i \omega_{2}\right)} .
\end{gathered}
$$

The sum over Matsubara frequencies yields

$$
\begin{array}{r}
u=U^{2} \sum_{\vec{k}}\left[\frac { f ( \epsilon _ { \vec { k } } ) } { i \omega _ { 4 } } \left(\frac{1}{\left(i \omega_{3}-\Delta \epsilon\right)\left(i \omega_{3}+i \omega_{2}-\Delta \epsilon\right)}-\right.\right. \\
\left.-\frac{1}{\left(i \omega_{1}+\Delta \epsilon\right)\left(i \omega_{1}+i \omega_{2}+\Delta \epsilon\right)}\right) \\
+\frac{f\left(\epsilon_{\vec{k}+\vec{Q}}\right)}{i \omega_{2}}\left(\frac{1}{\left(i \omega_{3}-\Delta \epsilon\right)\left(i \omega_{1}+i \omega_{2}+\Delta \epsilon\right)}-\right. \\
\left.\left.-\frac{1}{\left(i \omega_{1}+\Delta \epsilon\right)\left(i \omega_{3}+i \omega_{2}-\Delta \epsilon\right)}\right)\right]
\end{array}
$$

where $\Delta \epsilon=\epsilon_{\vec{k}}-\epsilon_{\vec{k}+\vec{Q}}$. Letting $\omega_{i, \pm}=\left(\omega_{i} \pm \omega_{i+2}\right) / 2$ $(i=1,2)$, and noting that $\omega_{1+}=-\omega_{2+}$, this simplifies to

$$
u=2 U^{2} \sum_{\vec{k}} \frac{\left(f\left(\epsilon_{\vec{k}+\vec{Q}}\right)-f\left(\epsilon_{\vec{k}}\right)\right) W_{-}}{\left(W_{-}^{2}+\omega_{1+}^{2}\right)\left(W_{-}^{2}+\omega_{2-}^{2}\right)},
$$

where

$$
W_{-}=\left(i \omega_{1-}+\Delta \epsilon\right)
$$

Thus in Matsubara frequency space, $u$ is largest for $\omega_{1+}=\omega_{2-}=0$, so it should indeed be reasonable to estimate it in that limit:

$$
u\left(i \omega_{1}, 0,0\right)=U^{2} \frac{\partial^{2}}{\partial\left(i \omega_{1}\right)^{2}} \sum_{\vec{k}} \frac{f\left(\epsilon_{\vec{k}+\vec{Q}}\right)-f\left(\epsilon_{\vec{k}}\right)}{i \omega_{1}+\Delta \epsilon} .
$$

In turn, it should be possible to approximate $u$, Eq. D20, by its $\omega_{1} \rightarrow 0$ limit, if this is nonsingular. From Eq. 4, $U \chi_{0}(\vec{Q}, \omega)=B \omega^{2}+i C \omega+1-\delta_{0}$. Thus, the analytic continuation $i \omega_{1} \rightarrow \omega+i \delta$ yields

$$
u(0,0,0)=U^{2} \lim _{\omega \rightarrow 0} \frac{\partial^{2} \chi_{0}(\vec{Q}, \omega)}{\partial \omega^{2}} \simeq 2 B U .
$$

Due to the plateau in $\chi(\vec{Q}, \omega), B$ (Table I) and hence $u$ are extremely small. The smallness of $u$ is true only in the limit that all external frequencies are small, which means that a more complicated expression should be used to evaluate $u$. Moreover, there is an additional problem: as found above, $B$ has a correction in $\ln (\omega)$, which would formally be divergent. Hence, the model is not fully selfconsistent, and $u$ will be treated as an empirical parameter. The weak logarithmic divergence will be neglected, and $u$ approximated by a constant.

\section{APPENDIX E: INTERLAYER COUPLING}

\section{Dispersion of $t_{z}$ : Direct and Staggered Stacking}

Andersen, et al. ${ }^{126}$ demonstrated that the anomalous form of interlayer hopping in the cuprates, $t_{z}=t_{z 0}\left(c_{x}-\right.$ $\left.c_{y}\right)^{2}$, could be understood by coupling the $\mathrm{Cu}_{d_{x}^{2}-d_{y}^{2}}$ and $\mathrm{O}_{p}$ orbitals to the $\mathrm{Cu}_{4 s}$ orbitals, which have significant 
interlayer coupling. Here, I provide a simplified calculation including only these orbitals, and show how the dispersion is modified by staggered stacking of the $\mathrm{CuO}_{2}$ layers. For uniform stacking $(\mathrm{Cu}$ above $\mathrm{Cu})$, the hopping matrix becomes

$$
H=\left(\begin{array}{cccc}
\Delta & -2 t s_{x} & 2 t s_{y} & 0 \\
-2 t s_{x} & 0 & 0 & -2 t_{p s} s_{x} \\
2 t s_{y} & 0 & 0 & -2 t_{p s} s_{y} \\
0 & -2 t_{p s} s_{x} & -2 t_{p s} s_{y} & \Delta_{s}+E_{s z}
\end{array}\right)
$$

with $s_{i}=\sin k_{i} a / 2$. Here the first (last) row is for the $\mathrm{Cu}_{d_{x}^{2}-d_{y}^{2}}\left(\mathrm{Cu}_{4 s}\right)$ orbital, and the middle rows are for the $\mathrm{O}_{p x}$ and $\mathrm{O}_{p y}$ orbitals, with $E_{s z}=-4 t_{s z} \cos k_{z} c$. In the limit $\Delta_{s}+E_{s z}>\Delta>>t, t_{p s}$, the antibonding band has dispersion

$$
E=\Delta-\frac{2 t^{2}}{\Delta}\left(c_{x}+c_{y}-2\right)-\frac{4 t^{2} t_{p s}^{2}}{\Delta^{2}\left(\Delta_{s}+E_{s z}\right)}\left(c_{x}-c_{y}\right)^{2}
$$

so if $t_{s z}<<\Delta_{s}$, the interlayer hopping has the form $t_{z 0} \cos k_{z} c\left(c_{x}-c_{y}\right)^{2}$, with $t_{z 0}=-16 t^{2} t_{p s}^{2} t_{s z} / \Delta^{2} \Delta_{s}^{2}$. While this form had been suggested earlier ${ }^{127}$ and found experimentally for the bilayer splitting in $\mathrm{BSCCO}^{128}$, it should be noted that it is only approximate, and that, at least in YBCO, there is considerable splitting of the bilayer bands along the zone diagonal ${ }^{126}$. Nevertheless, this form is adequate for the present purposes.

When successive layers are staggered, the only modification to the hopping matrix is in the form of $E_{s}\left(k_{z}\right)$, which now acquires an in-plane dispersion,

$$
\begin{array}{r}
E_{s}\left(k_{z}\right)=-4 t_{s z} \cos k_{z} c\left[\cos \left(k_{x}+k_{y}\right) a / 2+\cos \left(k_{x}-k_{y}\right) a / 2\right] \\
=-8 t_{s z} \cos k_{z} c \cos k_{x} a / 2 \cos k_{y} a / 2
\end{array}
$$

which leads to Eq. 65.

\section{Estimation of $t_{z}$ from Resistivity Anisotropy}

The dc conductivity can be estimated

$$
\sigma_{i i}=\frac{2 e^{2}}{\Omega} \sum_{\vec{k}} v_{i}^{2} \delta\left(\epsilon_{\vec{k}}-\mu\right) \tau_{\vec{k}}
$$

$i=x, y, z$, with $\Omega$ the unit cell volume, $v_{i}=\hbar^{-1} d \epsilon_{\vec{k}} / d k_{i}$, and $\tau_{\vec{k}}$ the scattering rate. Recent ARPES data suggest that, when bilayer splitting is resolved, $\tau_{\vec{k}}$ is relatively isotropic over the Fermi surface ${ }^{129}$. Taking $\tau_{\vec{k}}$ independent of $\vec{k}$, the conductivities are given by integrals over the Fermi surface. Figure 42 a shows a normalized conductivity ratio,

$$
\frac{\hat{\sigma}_{z z}}{\sigma_{x x}}=\frac{a t^{2}}{c t_{z 0}^{2}} \frac{\sigma_{z z}}{\sigma_{x x}}
$$

while Fig. $42 \mathrm{~b}$ shows the resulting normalized interlayer hopping $\hat{t}_{z 0}=t_{z 0} \sqrt{c / a}$, which would be required to produce a resistivity anisotropy $\rho_{z z} / \rho_{x x}=1000$. For simplicity, it is assumed that $t_{z 0}$ is small, and $\hat{\sigma}_{z z} / \sigma_{x x}$ is evaluated in the limit $t_{z 0} \rightarrow 0$. It can be seen that (a) the staggered stacking reduces the conductivity by approximately a factor of 20, independent of doping (except near the VHS), so (b) assuming the resistivity anisotropy is 1000 for optimally doped LSCO, it is estimated that $t_{z 0} / t=0.11$ for staggered stacking; by contrast, if the stacking had been uniform, a value of $t_{z 0} / t=0.025$ would have been required. This calculation has recently been extended ${ }^{130}$ to the bilayer compound $\mathrm{Bi}_{2} \mathrm{Sr}_{2} \mathrm{CaCu}_{2} \mathrm{O}_{8}$ (Bi2212), which has both uniform and staggered stacking. By comparison with a band structure calculation, the approximate values $t_{z} / t=0.38$ for the intracell hopping (corresponding to uniform stacking), and 0.14 for the intercell (staggered) hopping were found.

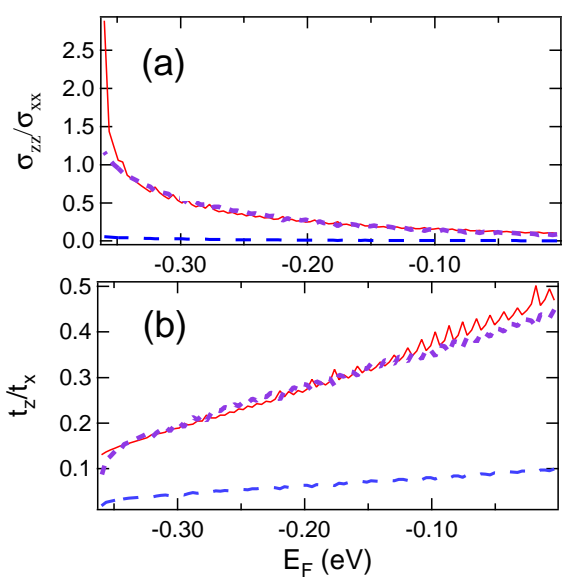

(E3)

FIG. 42. (a) Normalized conductivity ratio, $\hat{\sigma}_{z z} / \sigma_{x x}$ vs doping $E_{F}$, for uniform (solid line) and staggered stacking (long dashed line and short dashed line, $(\times 20)$ ); and (b) resulting normalized interlayer hopping $\hat{t}_{z 0}$ for staggered (solid line) and uniform stacking (long dashed line and short dashed line, $(\times 4.5)$.

\section{3. z-Component of Ordering Vector}

Given a finite interlayer hopping $t_{z}$, the first issue is to identify the three-dimensional ordering vector: what $Q_{z}$ minimizes the free energy? At mean field level, the initial magnetic instability will be associated with the state for which the RPA denominator first diverges, i.e., the state with the largest value of $\operatorname{Re} \chi_{0}\left(\vec{Q}, Q_{z}\right)$. (Note that these calculations implicitly assume that the two-dimensional ground state involves commensurate order at $\vec{Q}$.) For uniform stacking, a complicated dependence on doping, temperature, and $t_{z}$ is found. Figures 43,44 plot $\chi_{0}$ vs chemical potential for $T=100 \mathrm{~K}, 10 \mathrm{~K}$, respectively. The shift of the susceptibility peak with doping can readily 
be understood by comparison with Fig. 1. Both temperature and interlayer coupling act to smear out the VHS, and in both cases cause the susceptibility peak to shift to smaller chemical potential (lower hole doping), Fig. 43d. Note that the peak shifts at different rates for different $Q_{z}$-values, showing that the band is developing a considerable c-axis dispersion. The fastest shift (short dashed line in Fig. 43d, corresponding to $Q_{z}=0$ ) can thus be considered as representing a crossover from quasi-twodimensional to fully three dimensional dispersion.
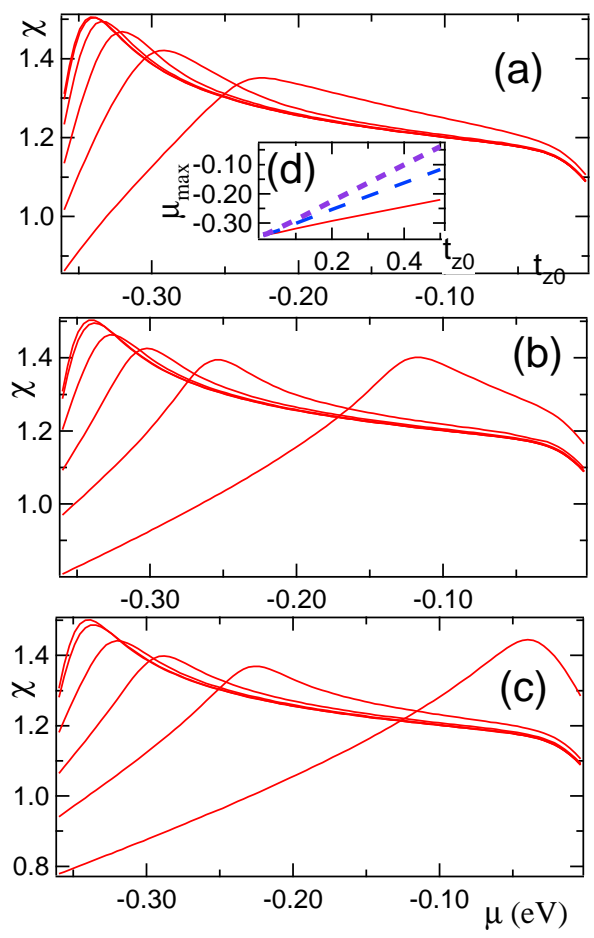

FIG. 43. $\chi_{0}\left(\vec{Q}, Q_{z}\right)$ at $T=100 K$ vs chemical potential $\mu$, for uniform stacking and $Q_{z}=\pi$ (a), $\pi / 2$ (b), and 0 (c). The various curves correspond to $t_{z 0} / t=0.01,0.02,0.05,0.1$, 0.2 , and 0.5 , with the peak in $\chi_{0}$ shifting to the right with increasing $t_{z 0}$. Inset (d): position of peak, $\mu_{\max }$, vs $t_{z 0}$ for $Q_{z}$ $=\pi$ (solid line), $\pi / 2$ (long dashed line), and 0 (short dashed line).
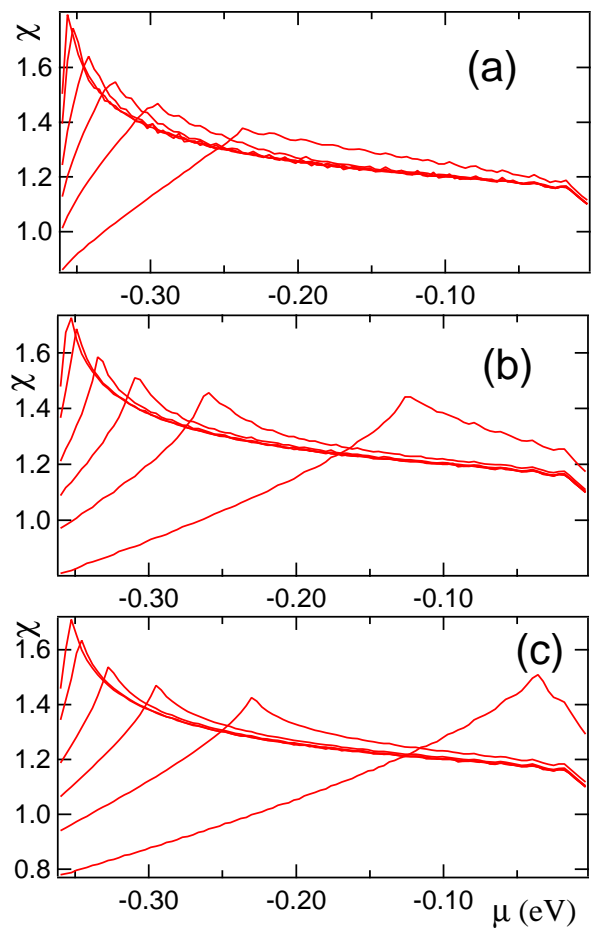

FIG. 44. $\chi_{0}\left(\vec{Q}, Q_{z}\right)$ vs chemical potential $\mu$, as in Fig. 43, but at $T=10 K$.

This dispersive shift of the peak in $\chi_{0}$ leads to a doping dependence of the optimal $Q_{z}$, as illustrated in Fig. 45 for $t_{z 0}=0.2 t$. For large hole doping, near the $t_{z 0}=0 \mathrm{VHS}$, the susceptibility maximum corresponds to $Q_{z}=\pi / c$, while near the susceptibility peak, the spin modulation becomes incommensurate (intermediate values of $Q_{z}$ have the largest susceptibility). There is a rapid evolution of the optimal $Q_{z}$, and beyond the peak regime, over essentially the entire electron-doped regime, the optimal $Q_{z}$ is 0 . This same pattern is repeated for smaller $t_{z 0}$, with only the region of the susceptibility peak changing. The results are essentially independent of the $\operatorname{sign}$ of $t_{z}$.
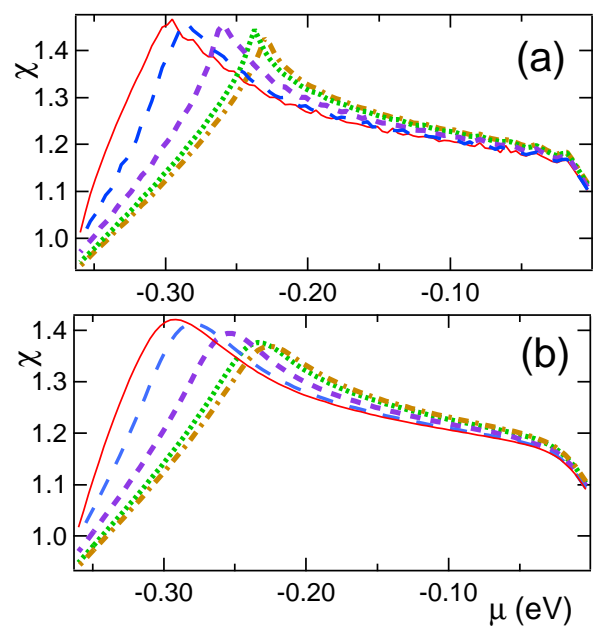
FIG. 45. $\chi_{0}\left(\vec{Q}, Q_{z}\right)$ vs chemical potential $\mu$, for uniform stacking and $t_{z 0}=0.2 t$, and $T=10 \mathrm{~K}$ (a), or $100 \mathrm{~K}(\mathrm{~b})$, with $Q_{z} / \pi=1$ (solid line), 0.75 (long dashed line), 0.5 (short dashed line), 0.25 (dotted line), 0 (dot-dashed line).

\section{Calculation of $A_{z}$}

\section{a. Uniform Stacking}

Given $t_{z}$ and $Q_{z}$, the parameter $A_{z}$ of Eq. 63 can be evaluated: $U \chi\left(\vec{Q}+q_{z} \hat{z}, \omega=0\right)=U \chi\left(\vec{Q}+Q_{z} \hat{z}, 0\right)+$ $A_{z}\left(q_{z}-Q_{z}\right)^{2}$. The dominant ordering vectors, $Q_{z}=\pi / c$ and $Q_{z}=0$, can be analyzed in more detail. For the former choice,

$$
\begin{aligned}
A_{z}^{\pi}= & \frac{U c^{2}}{4} \sum_{\vec{k}}\left[\frac { t _ { z } c _ { z } } { \epsilon _ { \vec { k } } - \epsilon _ { \vec { k } + \vec { Q } } + i \delta } \left(2 \frac{f_{\vec{k}}-f_{\vec{k}+\vec{Q}}}{\epsilon_{\vec{k}}-\epsilon_{\vec{k}+\vec{Q}}+i \delta}\right.\right. \\
& \left.\left.-\left[f_{\vec{k}}^{\prime}+f_{\vec{k}+\vec{Q}}^{\prime}\right]\right)-2 t_{z}^{2} s_{z}^{2}\left(\frac{f_{\vec{k}}^{\prime \prime}-f_{\vec{k}+\vec{Q}}^{\prime \prime}}{\epsilon_{\vec{k}}-\epsilon_{\vec{k}+\vec{Q}}+i \delta}\right)\right],
\end{aligned}
$$

with $f_{\vec{k}}^{\prime}=-f_{\vec{k}}\left(1-f_{\vec{k}}\right) / k_{B} T, f_{\vec{k}}^{\prime \prime}=-f_{\vec{k}}^{\prime}\left(1-2 f_{\vec{k}}\right) / k_{B} T$, $c_{z}=\cos k_{z} c, s_{z}=\sin k_{z} c$. For the latter case

$$
\begin{array}{r}
A_{z}^{0}=\frac{-U c^{2}}{4} \sum_{\vec{k}}\left[t_{z} c_{z}\left(\frac{f_{\vec{k}}^{\prime}-f_{\vec{k}+\vec{Q}}^{\prime}}{\epsilon_{\vec{k}}-\epsilon_{\vec{k}+\vec{Q}}+i \delta}\right)\right. \\
+2 t_{z}^{2} s_{z}^{2}\left[\frac{f_{\vec{k}}^{\prime \prime}-f_{\vec{k}+\vec{Q}}^{\prime \prime}}{\epsilon_{\vec{k}}-\epsilon_{\vec{k}+\vec{Q}}+i \delta}\right. \\
\left.+8 \frac{f_{\vec{k}}-f_{\vec{k}+\vec{Q}}}{\left(\epsilon_{\vec{k}}-\epsilon_{\vec{k}+\vec{Q}}+i \delta\right)^{3}}-4 \frac{f_{\vec{k}}^{\prime}+f_{\vec{k}+\vec{Q}}^{\prime}}{\left(\epsilon_{\vec{k}}-\epsilon_{\vec{k}+\vec{Q}}+i \delta\right)^{2}}\right] .
\end{array}
$$

Figure 46 ( 47a) shows how $\chi_{0}\left(\vec{Q}, Q_{z}\right)$ varies with $Q_{z}$ for $t_{z 0}=0.1 t(0.02 t)$, for a number of different dopings. For the entire electron-doped regime, the peak is at $Q_{z m}=0$ (Fig. 46b, 47d), crossing over to $Q_{z m}=\pi / c$ in the hole doped regime. Away from the peak, the susceptibility varies as $\hat{A}_{z} q_{z}^{2}$, with $q_{z}=Q_{z}-Q_{z m}$, and in the electron-doped regime the full variation can be approximated by a cosine. The amplitude of the cosine falls to zero as the C-point is approached. In the quasi-two-dimensional regime this amplitude scales with $t_{z 0}^{2}$. Figure $47 \mathrm{~b}, \mathrm{c}$ shows plots of the best parabolic fit to $A_{z}^{\prime}=\hat{A}_{z} / c^{2}$ for $t_{z 0} / t=0.02$ (squares) and 0.1 (triangles). For $t_{z 0} / t=0.1$, an alternative $A_{z}^{\prime}$ is shown, found by fitting the full susceptibility as a cosine in $q_{z}$ (circles). The good agreement between the two techniques shows that this is a reasonable approximation in the electron-doped regime $(-0.2 \mathrm{eV} \leq \mu \leq 0)$. Near the susceptibility peak, the variation is nonsinusoidal, and the parabolic fit leads to a large value for $A_{z}^{\prime}$.

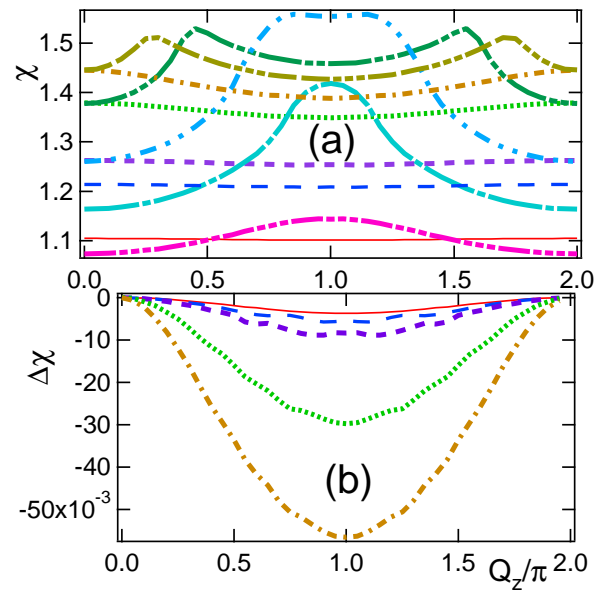

FIG. 46. (a) $\chi_{0}\left(\vec{Q}, Q_{z}\right)$ vs $Q_{z}$ for $t_{z 0}=0.1 t$, and $T=10 K$, and a variety of chemical potentials $\mu$ $=-0.003559$ (solid line), -0.08898 (long dashed line), -0.1779 (short dashed line), -0.2669 (dotted line), -0.2847 (dot-dashed line), -0.2954 (long-long-short-short-short dashed line), -0.3025 (long-short-short dashed line), -0.3203 (dash-dot-dot line), -0.3381 (long-short dashed line), and -0.3559 meV (long-short-short-short dashed line). $\Delta \chi=\chi_{0}\left(\vec{Q}, Q_{z}\right)-\chi_{0}\left(\vec{Q}, Q_{z}=0\right)$, where the curves have the same meaning as in frame (a).
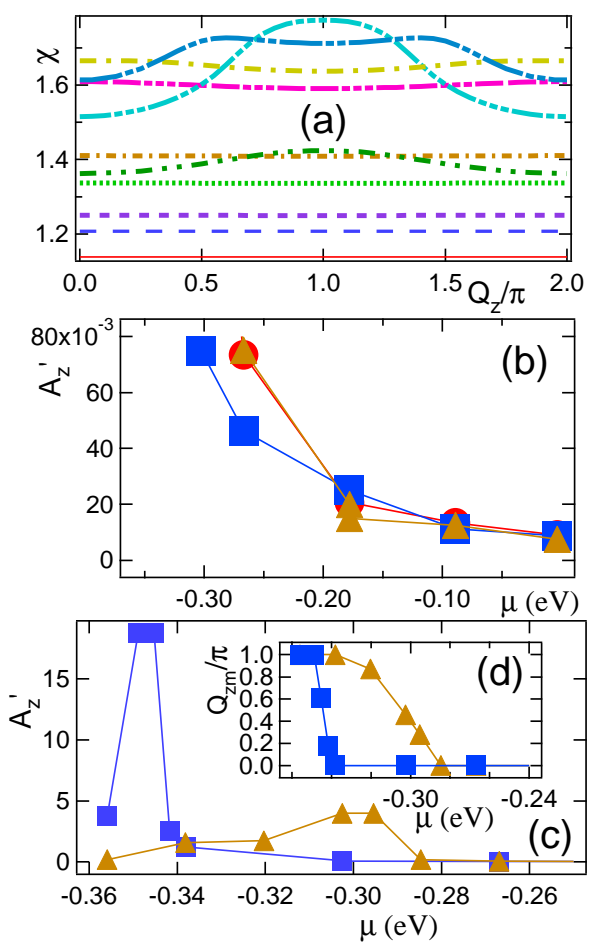
FIG. 47. (a) $\chi_{0}\left(\vec{Q}, Q_{z}\right)$ vs $Q_{z}$ for $t_{z 0}=0.02 t$, and $T=10 K$, and a variety of chemical potentials $\mu=-0.003559$ (solid line), -0.08898 (long dashed line), -0.1779 (short dashed line), -0.2669 (dotted line), -0.3025 (dot-dashed line), -0.3381 (long-long-short-short-short dashed line), -0.3417 (long-dashed-dotted line), -0.3452 (long-short-short dashed line), -0.3488 (long-short-short-short dashed line), and -0.3559 meV (long-dash-dot-dotted line). (b,c) $A_{z}^{\prime}=A_{z} / U c^{2}$ vs $\mu$ for $t_{z 0} / t=0.02$ (squares, $\left.A_{z}^{\prime} \times 25\right)$ and 0.1 (triangles,circles). (d) $Q_{z m}$ vs $\mu$ for $t_{z 0} / t=0.02$ (squares) and 0.1 (triangles).

\section{b. Staggered Stacking}

The same calculations can be repeated for the $t_{z}$ of Eq. 65, associated with staggered stacking; Fig. 48a shows $A_{z}$ calculated from Eqs. E6, E7 at $Q_{z}=0$ (solid lines) and $\pi$ (dashed lines). The frustration induced by staggering of the $\mathrm{CuO}_{2}$ layers is reflected in a strong suppression of the $q_{z}$-dependence of $\chi$, which leaves a small residual contribution quadratic in $t_{z 0}$, Fig. $48 \mathrm{~b}$. Since $t_{z}$ vanishes at $(\pi, 0)$, there is no shift of the susceptibility peak with doping. Note the symmetry of the $A_{z}$ values between 0 and $\pi$. In fact, $\chi\left(Q_{z}\right)$ is closely sinusoidal, particularly for small $t_{z 0}$, with maxima either at $\pi$ or 0 . Thus, near either the $\mathrm{H}$ - or C-points, the maximum of $\chi$ corresponds to $Q_{z}=\pi$. For intermediate dopings, $Q_{z}=0$ is favored. At two distinct chemical potentials, the amplitude of the cosine collapses and changes sign. At the crossing points, $\chi$ is independent of $Q_{z}$, leading formally to $T_{N} \rightarrow 0$. Note from Fig. 48c that the suppression of $A_{z}$ is approximately in the same ratio as that of the resistivity, found above.

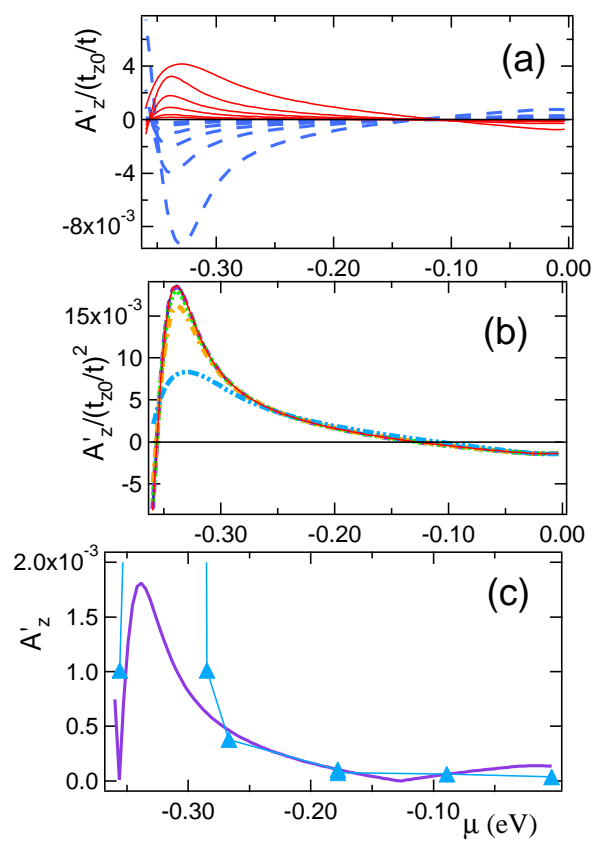

FIG. 48. (a) $A_{z}^{\prime}=A_{z} / U c^{2}$ vs chemical potential $\mu$ for $Q_{z}=$ 0 (solid lines) or $\pi$ (dashed lines), for a variety of values of $t_{z 0}$ and $T=100 \mathrm{~K}$. In order of increasing amplitude, the values are $t_{z 0} / t=0.01,0.02,0.05,0.1,0.2$, and 0.5. (b) Scaling of $A_{z}^{\prime(0)}$ with $\left(t_{z 0} / t\right)^{2}$. Curves are $t_{z 0} / t=0.01$ (solid line), 0.02 (long dashed line), 0.05 (short dashed line), 0.1 (dotted line), 0.2 (dot-dashed line), and 0.5 (dot-dot-dashed line). (c) Comparison of $\max \left(A_{z}\right)$ for staggered stacking (solid line) and uniform stacking (triangles, $\times 1 / 20$ ) at $t_{z 0} / t=0.1$.

\section{Calculation of $T_{N}$}

When there is a finite interlayer hopping $t_{z}$, Eq.13 becomes

$$
\begin{aligned}
\delta-\bar{\delta}_{0}=\frac{6 u T a^{2} c}{\pi^{2} A} \int_{0}^{\frac{\pi}{c}} \frac{d q_{z}}{\pi} \int_{y_{0}}^{y_{0}+A q_{c}^{2}} & \frac{d y}{y} \tan ^{-1}\left(\frac{2 T C}{y}\right) \\
& \left.\simeq \frac{3 u T a^{2}}{\pi A} \ln \left(\frac{T}{T_{3 D}}\right)\right],
\end{aligned}
$$

where $y_{0}=\delta+A_{z} q_{z}^{\prime 2}$ and $T_{3 D}=\pi^{2} A_{z} / 2 C e^{2} c^{2}$. (A small correction to $\bar{\delta}_{0}$ is neglected. Treating the $q_{z}$ dependence as a cosine rather than a cutoff quadratic leads to qualitatively similar results.) Thus a finite $A_{z}$ always cuts off the divergence found in Eq.13, leading to a finite $T_{N}$ whenever there is a zero-temperature Neel state (e.g., up to a QCP). It should be noted that the above calculation implicitly assumed that $T>T_{3 D} \sim A_{z}$ : for $T<T_{3 D}$ the logarithm is cut off and the system behaves like an anisotropic three-dimensional magnet. For $t_{z 0} / t<0.1$, the system is generally in the quasi-twodimensional limit, Fig. 49a. Figure 49b compares the mean-field Neel transition with the Neel transition found assuming uniform stacking and finite interlayer couplings $t_{z 0} / t=0.1,0.02$, and $2 \times 10^{-6}$ the last found by scaling the $T_{3 D}$ for $t_{z 0} / t=0.02$ by the ratio of $t_{z 0}^{2}$ 's]. It is seen that $T_{N} \rightarrow 0$ as $t_{z 0} \rightarrow 0$, albeit exceedingly slowly.

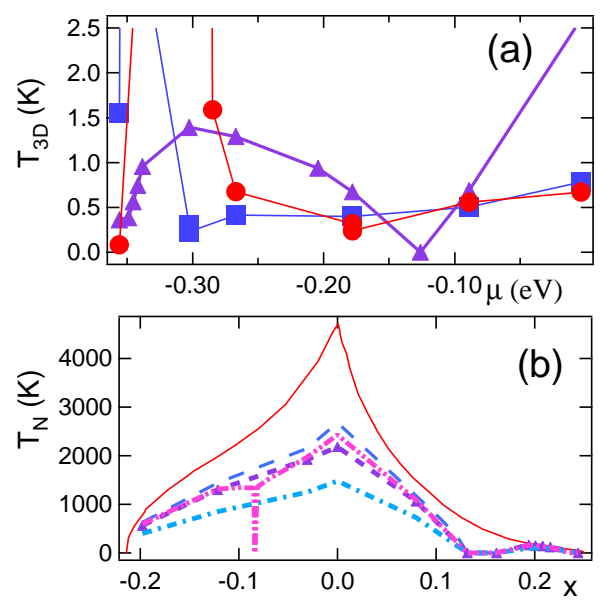


FIG. 49. (a) $T_{3 D}$ vs $\mu$ for $T=10 K$ and uniform stacking with $t_{z 0}=0.1 t$ (circles, $\times 1 / 25$ ) or 0.02 (squares), or staggered stacking with $t_{z 0}=0.02$ (triangles). (b) $T_{N}$ vs $x$, comparing mean field transition (solid line) with interlayer coupling models (uniform stacking) assuming $t_{z 0} / t=0.1$ (long dashed line), 0.02 (short dashed line), and $2 \times 10^{-6}$ (dot-dashed line), and the staggered stacking model assuming $t_{z 0} / t=0.1$ (dot-dot-dash line).

The above calculations are for uniform stacking. For staggered stacking $A_{z}$ is reduced, in approximately the same ratio as the resistivities. Hence, the staggered stacking with $t_{z 0} / t=0.1$ should be comparable to uniform stacking with $t_{z 0} / t=0.02$, as observed, Fig. 49 . While $T_{N}$ technically goes to zero for staggered stacking near $x=-0.0838$, the decrease is logarithmic, and in practice no more than a weak dip is expected to be observed (the point with $T_{N}=0 K$ is omitted from the plot in Fig. 31). Hence, if $t_{z 0}$ is estimated from the resistivity, it will be nearly impossible to distinguish uniform from staggered stacking via measurements of $T_{N}$.

In the above calculations, a constant value of $A$ was assumed for each doping, as given in Fig. 9. In fact, for the electron-doped cuprates, $A \sim 1 / T^{1.5}$ for $T>T_{A}^{*}$, Fig. 8. This would cause an enhancement of the logarithmic correction, $\sim T^{2.5}$, tending to pin $T_{N}$ close to $T_{A}^{*}$. For the present parameter values, this could reduce $T_{N}$ by roughly a factor of two, still larger than the experimental values.

A more likely source of the discrepancy is the possible temperature dependence of $U_{\text {eff }}$, Appendix B. The large $U_{\text {eff }}$ at half filling arises from lack of screening, in the presence of a Mott gap - and is appropriate in analyzing the low- $T$ Fermi surfaces found in ARPES. For calculating the onset of the Mott gap, the mean field $T_{N}$, it is more appropriate to use the paramagnetic susceptibility, as in Fig 37a. When this is done, considerably smaller transition temperatures are found, both at the mean field level, Fig. 50a, and when fluctuations and interlayer hopping are included, Fig. 50b. While the latter are closer to the experimental values, no attempt has been made to correct $U_{e f f}$ for the short range gap.

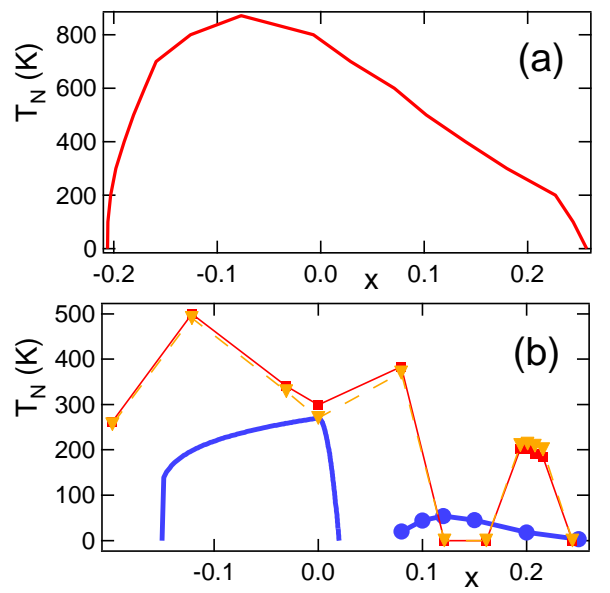

FIG. 50. (a) Mean field $T_{N}$ vs $x$ assuming paramagnetic $U_{\text {eff }}$ (Appendix B). (b) Corresponding $T_{N}$ vs $x$, calculated using Eq. E8. Squares $=$ staggered stacking with $t_{z 0} / t=0.1$; triangles $=$ uniform stacking with $t_{z 0} / t=0.02$; solid line and circles = data, as in Fig. 31 .

${ }^{1}$ J.R. Schrieffer, X.G. Wen, and S.C. Zhang, Phys. Rev. B39, 11663 (1989).

${ }^{2}$ A.P. Kampf and J.R. Schrieffer, Phys. Rev. B42, 7967 (1990).

${ }^{3}$ Y.M. Vilk and A.-M.S. Tremblay, J. Phys. I 7, 1309 (1997); B. Kyung, J.S. Landry, D. Poulin, and A.-M.S. Tremblay, Phys. Rev. Lett. 90, 099702 (2003).

${ }^{4}$ N.E. Bickers, D.J. Scalapino, and S.R. White, Phys. Rev. Lett. 62, 961 (1989).

${ }^{5}$ H. Schulz, J. Phys. (Paris) 50, 2833 (1989); D. Poilblanc and T.M. Rice, Phys. Rev. B39, 9749 (1989); J. Zaanen and O. Gunnarsson, ibid. B40, 7391 (1989); B.I. Shraiman and E.D. Siggia, Phys. Rev. Lett. 62, 1564 (1989).

${ }^{6}$ H.J. Schulz, Phys. Rev. Lett. 65, 2462 (1990); C. Zhou and H.J. Schulz, Phys. Rev. B52, 11557 (1995).

${ }^{7}$ R.S. Markiewicz, Mod. Phys. Letts. B1, 187 (1987); R.S. Markiewicz, J. Phys. Cond. Matt. 2, 665 (1990).

${ }^{8}$ N.P. Armitage, F. Ronning, D.H. Lu, C. Kim, A. Damascelli, K.M. Shen, D.L. Feng, H. Eisaki, Z.-X. Shen, P.K. Mang, N. Kaneko, M. Greven, Y. Onose, Y. Taguchi, and Y. Tokura, Phys. Rev. Lett. 88, 257001 (2002).

${ }^{9}$ C. Kusko, R.S. Markiewicz, M. Lindroos, and A. Bansil, Phys. Rev. B66, 140513 (2002).

${ }^{10}$ R.B. Laughlin and D. Pines, Proc. Nat. Acad. Sci. 97, 28 (2000).

${ }^{11}$ T. Moriya, "Spin Fluctuations in Electron Magnetism", (Springer, Berlin, 1985).

12 R.S. Markiewicz, in "Intrinsic Multiscale Structure and Dynamics in Complex Electronic Oxides", edited by A.R. Bishop, S.R. Shenoy, and S. Sridhar, World Scientific (2003), p. 109 (cond-mat/0308361).

${ }^{13}$ H. Kusunose and T.M. Rice, Phys. Rev. Lett. 91, 186407 (2003).

${ }^{14}$ D. Sénéchal and A.-M.S. Tremblay, cond-mat/0308625.

${ }^{15}$ J. Kanamori, Prog. Theor. Phys. 30, 275 (1963).

${ }^{16}$ K. K. Murata and S. Doniach, Phys. Rev. Lett. 29, 285 (1972).

${ }^{17}$ H. Hasegawa and T. Moriya, J. Phys. Soc. Jpn. 36, 1542 (1974).

${ }^{18}$ For recent reviews, see T. Moriya and K. Ueda, Adv. Phys. 49, 555 (2000); and T. Moriya, Acta Physica Polonica B34, 287 (2003) (cond-mat/0207669) (Proceedings of the Strongly Correlated Electron Systems [SCES'02] conference in Krakow/Poland).

${ }^{19}$ P.A. Lee, T.M. Rice, and P.W. Anderson, Sol. St. Commun. 14, 703 (1974).

${ }^{20}$ R.S. Markiewicz, Physica C169, 63 (1990).

${ }^{21}$ W. Götze L. and Sjögren, Rep. Prog. Phys. 55, 241 (1992). 
22 S. Andergassen, S. Caprara, C. Di Castro, M. Grilli, Phys. Rev. Lett. 87, 056401 (2001).

${ }^{23}$ S. Onoda and M. Imada, J. Phys. Soc. Jpn. 68, 2762 (1999).

24 N.M.R. Peres and M.A.N. Araújo, Phys. Stat. Sol. 236, 523 (2003).

${ }^{25}$ H.M. Rønnow, D.F. McMorrow, R. Coldea, A. Harrison, I.D. Youngson, T.G. Perring, G. Aeppli, O. Syljuåsen, K. Lefmann, and C. Rischel, Phys. Rev. Lett. 87, 37202 (2001).

${ }^{26}$ P. Fulde, "Electron Correlations in Molecules and Solids" (2d Ed.) (Springer, Berlin, 1993).

27 N. Nagaosa, "Quantum Field Theory in Strongly Correlated Electronic Systems", (Springer, Berlin, 1999), Ch. 3.

${ }^{28}$ P. Monthoux and D. Pines, Phys. Rev. B47, 6069 (1993); B.P. Stojković and D. Pines, Phys. Rev. B55, 8576 (1997).

${ }^{29}$ S. Chakravarty, B.I. Halperin, and D.R. Nelson, Phys. Rev. Lett. 60, 1057 (1988).

${ }^{30}$ P. Kopietz and S. Chakravarty, Phys. Rev. B40, 4858 (1989).

31 P. Hasenfratz and F. Niedermayer, Phys. Lett. B268, 231 (1991).

32 T. Moriya, Y. Takahashi, and K. Ueda, J. Phys. Soc. Jpn., 59, 2905 (1990).

${ }^{33}$ A. Abanov, A.V. Chubukov, and J. Schmalian, Adv. Phys. 52, 119 (2003).

34 A.V. Chubukov, D. Pines, and J. Schmalian, in 'The Physics of Superconductors, Vol. I: Conventional and High-Tc Superconductors' edited by K.H. Bennemann and J.B. Ketterson (Springer-Verlag, Berlin, 2003), p. 495.

35 J.A. Hertz, Phys. Rev. B14, 1165 (1976).

36 A.J. Millis, Phys. Rev. B48, 7183 (1993).

${ }^{37}$ R.S. Markiewicz and S.M. Kelso, Solid State Commun. 25, 275 (1978).

${ }^{38}$ G. Kirczenow and K.S. Singwi, Phys. Rev. B19, 2117 (1979); T.L. Reinecke and S.C. Ying, Phys. Rev. Lett. 43, 1054 (1979).

${ }^{39}$ L.M. Sander, J.H. Rose, and H.B. Shore, Phys. Rev. B21, 2739 (1980).

${ }^{40}$ P. Bénard, L. Chen, and A.-M.S. Tremblay, Phys. Rev. B47, 15217 (1993).

${ }^{41}$ Q. Si, Y. Zha, K. Levin, and J.P. Lu, Phys. Rev. B47, 9055 (1993).

42 M. Lavagna and G. Stemmann, Phys. Rev. B49, 4235 (1994).

${ }^{43}$ F. Onufrieva, P. Pfeuty, and M. Kiselev, Phys. Rev. Lett. 82, 2370 (1999); F. Onufrieva and P. Pfeuty, Phys. Rev. B61, 799 (2000).

${ }^{44}$ V.M. Krasnov, Phys. Rev. B65, 140504 (2002).

${ }^{45}$ N. Harima, J. Matsuno, A. Fujimori, Y. Onose, Y. Taguchi, and Y. Tokura, Phys. Rev. B64, 220507 (2001).

${ }^{46}$ N. Ichikawa, S. Uchida, J.M. Tranquada, T. Niemöller, P.M. Gehring, S.-H. Lee, and J.R. Schneider, Phys. Rev. Lett. 85, 1738 (2000).

${ }^{47}$ R.S. Markiewicz, Physica C217, 381 (1993).

${ }^{48}$ P. Bourges, L.P. Regnault, J.Y. Henry, C. Vettier, Y. Sidis, and P. Burlet, Physica B215, 30 (1995).

49 A.V. Balatsky and P. Bourges, Phys. Rev. Lett. 82, 5337 (1999).
${ }^{50}$ B. Keimer, N. Belk, R.J. Birgeneau, A. Cassanho, C.Y. Chen, M. Gerven, and M.A. Kastner, Phys. Rev. B46, 14034 (1992).

${ }^{51}$ J.V. Alvarez, J. González, F. Guinea, and M.A.H. Vozmediano, cond-mat/9804153, to be published, Phys. Rev. B.

${ }^{52}$ S. Sachdev, A.V. Chubukov, and A. Sokol, Phys. Rev. B51, 14874 (1995).

${ }^{53}$ N. Bulut, D.J. Scalapino, and S.R. White, Phys. Rev. B47, 2742 (1993).

${ }^{54}$ C. Kusko and R.S. Markiewicz, unpublished.

55 A.V. Chubukov and D.K. Morr, Phys. Rev. B57, 5298 (1998), and Phys. Report 288, 355 (1997).

56 J. Schmalian, D. Pines, and B. Stojković, Phys. Rev. B60, 667 (1999).

${ }^{57}$ C. Kim, F. Ronning, A. Damascelli, D.L. Feng, Z.-X. Shen, B.O. Wells, Y.J. Kim, R.J. Birgeneau, M.A. Kastner, L.L. Miller, H. Eisaki, and S. Uchida, Phys. Rev. B65, 174516 (2002).

${ }^{58}$ R. Hlubina and T.M. Rice, Phys. Rev. B51, 9253 (1995); N.P. Armitage, et al., Phys. Rev. Lett. 87,147003 (2001).

${ }^{59}$ A. Ramšak, I. Sega, and P. Prelovšek, Phys. Rev. B61, 4389 (2000).

${ }^{60}$ Vilk and Tremblay ${ }^{3}$ have cautioned that a naive extension of their conserving approximation to include SCBA corrections could lead to conservation law violations.

${ }^{61}$ Z. Liu and E. Manousakis, Phys. Rev. B44, 2414, (1991), and Phys. Rev. B45, 2425 (1992); E. Dagotto, Rev. Mod. Phys. 66, 763 (1994).

${ }^{62}$ G. Martínez and P. Horsch, Phys. Rev. B44, 317 (1991).

${ }^{63}$ A.V. Chubukov and D.M. Frenkel, Phys. Rev. B46, 11884 (1992).

${ }^{64}$ S. LaRosa, I. Vobornik, F. Zwick, H. Berger, M. Grioni, G. Margaritondo, R.J. Kelley, M. Onellion, and A. Chubukov, Phys. Rev. B56, R525 (1997).

${ }^{65}$ C. Dürr, S. Legner, R. Hayn, S.V. Borisenko, Z. Hu, A. Theresiak, M. Knupfer, M.S. Golden, J. Fink, F. Ronning, Z.-X. Shen, H. Eisaki, S. Uchida, C. Janowitz, R. Mller, R.L. Johnson, K. Rossnagel, L. Kipp, and G. Reichardt, Phys. Rev. B63, 014505 (2001).

${ }^{66}$ F. Ronning, C. Kim, K.M. Shen, N.P. Armitage, A. Damascelli, D.H. Lu, D.L. Feng, Z.-X. Shen, L.L. Miller, Y.-J. Kim, F. Chou, and I. Terasaki, Phys. Rev. B67, 035113 (2003).

67 T.D. Stanescu and P. Phillips, Phys. Rev. Lett. 91, 017002 (2003), and cond-mat/0301254.

${ }^{68}$ R. S. Markiewicz, Phys. Rev. B62, 1252 (2000); M. Moraghebi, S. Yunoki, and A. Moreo, Phys. Rev. B63, 214513 (2001); F. Ronning, T. Sasagawa, Y. Kohsaka, K.M. Shen, A. Damascelli, C. Kim, T. Yoshida, N.P. Armitage, D.H. Lu, D.L. Feng, L.L. Miller, H. Takagi, and Z.-X. Shen, Phys. Rev. B67, 165101 (2003).

${ }^{69}$ A. Ino, C. Kim, M. Nakamura, T. Yoshida, T. Mizokawa, A. Fujimori, Z.-X. Shen, T. Kakeshita, H. Eisaki, and S. Uchida, Phys. Rev. B65, 094504 (2002).

${ }^{70}$ P.B. Visscher, Phys. Rev. B10, 943 (1974); E.L. Nagaev, "Physics of Magnetic Semiconductors" (Moscow, Mir, 1983); L.B. Ioffe and A.I. Larkin, Phys. Rev. B37, 5730 (1988)

${ }^{71}$ V.J. Emery, S.A. Kivelson, and H.Q. Lin, Phys. Rev. Lett. 
64, 475 (1990).

72 R.S. Markiewicz, Phys. Rev. Lett. 89, 229703 (2002).

${ }^{73}$ N. Miyakawa, P. Guptasarma, J.F. Zasadzinski, D.G. Hinks, and K.E. Gray, Phys. Rev. Lett. 80, 157 (1998).

${ }^{74}$ K.M. Lang, V. Madhavan, J.E. Hoffman, E.W. Hudson, H. Eisaki, S. Uchida, and J.C. Davis, Nature 415, 412 (2002).

75 J.L. Tallon, J.W. Loram, G.V.M. Williams, J.R. Cooper, I.R. Fisher, J.D. Johnson, M.P. Staines, and C. Bernhard, Phys. Stat. Sol. b215, 531 (1999).

${ }^{76}$ R.S. Markiewicz, J. Phys. Chem. Sol. 58, 1179 (1997).

${ }^{77}$ G.V.M. Williams, J.L. Tallon, R. Michalak, and R. Dupree, Phys. Rev. B57, 8696 (1998).

${ }^{78}$ D. Van der Marel, H.J.A. Molegraaf, J. Zaanen, Z. Nussinov, F. Carbone, A. Damascelli, H. Eisaki, M. Greven, P. H. Kes, and M. Li, Nature 425, 271 (2003).

${ }^{79}$ F.F. Balakirev, J.B. Betts, A. Migliori, S. Ono, Y. Ando, and G.S. Boebinger, Nature 424, 912 (2003).

${ }^{80}$ R.S. Markiewicz, unpublished.

${ }^{81}$ S. Ouazi, J. Bobroff, H. Alloul, and W.A. MacFarlane, cond-mat/0307728.

${ }^{82}$ A.J. Millis, H. Monien, and D. Pines, Phys. Rev. B42, 167 (1990); V. Barzykin and D. Pines, Phys. Rev. B52, 13585 (1995).

83 P.K. Mang, O.P. Vajk, A. Arvanitaki, J.W. Lynn, and M. Greven, cond-mat/0307093.

${ }^{84}$ Y.R. Wang, Phys. Rev. B43, 3786 (1991); C.-M. Ho, V.N. Muthukumar, M. Ogata, and P.W. Anderson, Phys. Rev. Lett. 86, 1626 (2001).

${ }^{85}$ J.M. Tranquada, B.J. Sternlieb, J.D. Axe, Y. Nakamura, and S. Uchida, Nature 375, 561 (1995); J.M. Tranquada, J.D. Axe, N. Ichikawa, A.R. Moodenbaugh, Y. Nakamura, and S. Uchida, Phys. Rev. Lett 78, 338 (1997).

${ }^{86}$ M. Matsuda, M. Fujita, K. Yamada, R.J. Birgeneau, Y. Endoh, and G. Shirane, Phys. Rev. B65, 134515 (2002).

${ }^{87}$ A.W. Hunt, P.M. Singer, A.F. Cederström, and T. Imai, Phys. Rev. B64, 134525 (2001).

${ }^{88}$ K. Yonemitsu, A.R. Bishop, and J. Lorenzana, Phys. Rev. B47, 12059 (1993).

${ }^{89}$ M. Matsuda, Y. Endoh, K. Yamada, H. Kojima, I. Tanaka, R.J. Birgeneau, M.A. Kastner, and G. Shirane, Phys. Rev. B45, 12548 (1992).

${ }^{90}$ A.H. Castro Neto and D. Hone, Phys. Rev. Lett. 76, 2165 (1996).

91 Y. Onose, Y. Taguchi, T. Ishikawa, S. Shinomori, K. Ishikaza, and Y. Tokura, Phys. Rev. Lett. 82, 5120 (1999).

${ }^{92}$ M. Rosseinsky, K. Prasseides, and P. Day, Inorg. Chem. 30, 2680 (1991).

${ }^{93}$ M. Fujita, K. Yamada, H. Hiraka, P.M. Gehring, S.H. Lee, S. Wakimoto, and G. Shirane, Phys. Rev. B65, 064505 (2002).

${ }^{94}$ H.A. Mook, Pengcheng Dai, S.M. Hayden, G. Aeppli, T.G. Perring, F. Dogan, Nature 395, 580 (1998).

${ }^{95}$ D. Reznik, P. Bourges, L. Pintschovius, Y. Endoh, Y. Sidis, Y. Shiokara, and S. Tajima, cond-mat/0307591.

${ }^{96}$ M. Eschrig and M.R. Norman, Phys. Rev. B67, 144503 (2003).

97 C. Pinettes and C. Lacroix, J. Phys. Cond. Matt. 6, 10093 (1994).

${ }^{98}$ H.Q. Ding, Phys. Rev. Lett. 68, 1927 (1992).
${ }^{99}$ A. Singh and Z. Tešanović, Phys. Rev. B43, 11445 (1991).

${ }^{100}$ B. Keimer, A. Aharony, A. Auerbach, R.J. Birgeneau, A. Cassanho, Y. Endoh, R.W. Erwin, M.A. Kastner, and G. Shirane, Phys. Rev. B45, 7430 (1992).

101 A.A. Katanin and A.P.Kampf, Phys. Rev. B66, 100403(R) (2002).

102 A. Singh, Z. Tešanović, H. Tang, G. Xiao, C.L. Chien, and J.C. Walker, Phys. Rev. Lett. 64, 2571 (1990).

103 R.J. Birgeneau, H.J. Guggenheim, and G. Shirane, Phys. Rev. B1, 2211 (1970).

${ }^{104}$ M. Fujita, T. Kubo, S. Kuroshima, T. Uefuji, K. Kawashima, K. Yamada, I. Watanabe, and K. Nagamine, Phys. Rev. B67, 014514 (2003).

${ }^{105}$ F. Kagawa, T. Itou, K. Miyagawa, and K. Kanoda, condmat/0307304.

106 P. Anderson, Bull. A.P.S. 48, 535 (2003).

107 A.A. Aligia, Phys. Rev. B39, 6700 (1989); Q. Si, Y. Zha, K. Levin, and J. Lu, Phys. Rev. B47, 9055 (1993); M. Lavagna and G. Stemmann, Phys. Rev. B49, 4235 (1994).

108 P. Sen and A. Singh, Phys. Rev. B48, 15792 (1993).

109 T.K. Lee, C.-M. Ho, and N. Nagaosa, Phys. Rev. Lett. 90, 067001 (2003).

${ }^{110}$ Ar. Abanov, A.V. Chubukov, and J. Schmalian, Europhys. Lett. 55, 369 (2001).

111 A.V. Chubukov, D.K. Morr, and K.A. Shakhnovich, Phil. Mag. B74, 563 (1996).

112 M.V. Sadovskii, Zh. Eksp. Teor. Fiz. 77, 2070 (1979) [Sov. Phys. JETP 50, 989 (1979)].

${ }^{113}$ Y. Yanase and K. Yamada, J. Phys. Soc. Jpn. 70, 1659 (2001); Y. Yanase, J. Phys. Soc. Jpn. 71, 278 (2002); Y. Yanase, T. Jujo, T. Nomura, H. Ikeda, T. Hotta, and K. Yamada, cond-mat/0309094.

114 Ar. Abanov, A.V. Chubukov, M. Eschrig, M.R. Norman, and J. Schmalian, Phys. Rev. Lett. 89, 177002 (2002).

${ }^{115}$ Y. M. Vilk, Liang Chen, and A.M. S. Tremblay,Phys.Rev. B49, 13267 (1994).

${ }^{116}$ K. Haule, A. Rosch, J. Kroha, and P. Wölfle, Phys. Rev. Lett. 89, 236402 (2002).

117 S. Sachdev, Phys. Rev. B59, 14054 (1999).

118 B. Kyung, V. Hankevych, A.-M. Daré, and A.-M.S. Tremblay, cond-mat/0312499.

${ }^{119}$ F.C. Zhang and T.M. Rice, Phys. Rev. B37, 3759 (1988).

120 M.S. Hybertsen, M. Schluter, and N.E. Christensen, Phys. Rev. B39, 9028 (1989).

${ }^{121}$ S. Moukouri, S. Allen, F. Lemay, B. Kyung, D. Poulin, Y.M. Vilk, and A.-M.S. Tremblay, Phys. Rev. B61, 7887 (2000).

${ }^{122}$ L. Chen, C. Bourbonnais, T. Li, and A.-M.S. Tremblay, Phys. Rev. Lett. 66, 369 (1991).

123 G. Esirgen, H.-B. Schüttler, C. Gröber, and H.G. Evertz, Phys. Rev. B64, 195105 (2001).

${ }^{124}$ I.S. Gradshteyn and I.M. Ryzhik, Table of Intrgrals, Series, and Products, 6th Ed. (Academic Press, San Diego, 2000), p. 883.

${ }^{125}$ F. Guinea, R.S. Markiewicz, and M.A.H. Vozmediano, cond-mat/0206208.

${ }^{126}$ O.K. Andersen, A.I. Liechtenstein, O. Jepsen, and F. Paulsen, J. Phys. Chem. Solids 56, 1573 (1995).

127 S. Chakravarty, A. Sudbo, P.W. Anderson, and S. Strong, Science 261, 337 (1993). 
${ }^{128}$ D.L. Feng, N.P. Armitage, D.H. Lu, A. Damascelli, J.P. $\mathrm{Hu}$, P. Bogdanov, A. Lanzara, F. Ronning, K.M. Shen, H. Eisaki, C. Kim, J.-i. Shimoyama, K. Kishio, and Z.-X. Shen, Phys. Rev. Lett. 86, 5550 (2001); Y.-D. Chuang, A.D. Gromko, A. Fedorov, Y. Aiura, K. Oka, Y. Ando, H. Eisaki, S.I. Uchida, and D.S. Dessau, Phys. Rev. Lett. 87, 117002 (2001).

129 P.V. Bogdanov, A. Lanzara, X.J. Zhou, W.L. Yang, H. Eisaki, Z. Hussain, and Z.X. Shen, Phys. Rev. Lett. 89, 167002 (2002).

${ }^{130}$ M. Lindroos, S. Sahrakorpi, R.S. Markiewicz, and A. Bansil, unpublished. 